\title{
WestVirginiaUniversity
}

THE RESEARCH REPOSITORY @ WVU

Graduate Theses, Dissertations, and Problem Reports

2015

\section{Fluoride Thin Films: from Exchange Bias to Multferroicity}

Trent A. Johnson

Follow this and additional works at: https://researchrepository.wvu.edu/etd

\section{Recommended Citation}

Johnson, Trent A., "Fluoride Thin Films: from Exchange Bias to Multferroicity" (2015). Graduate Theses, Dissertations, and Problem Reports. 5896.

https://researchrepository.wvu.edu/etd/5896

This Dissertation is protected by copyright and/or related rights. It has been brought to you by the The Research Repository @ WVU with permission from the rights-holder(s). You are free to use this Dissertation in any way that is permitted by the copyright and related rights legislation that applies to your use. For other uses you must obtain permission from the rights-holder(s) directly, unless additional rights are indicated by a Creative Commons license in the record and/ or on the work itself. This Dissertation has been accepted for inclusion in WVU Graduate Theses, Dissertations, and Problem Reports collection by an authorized administrator of The Research Repository @ WVU.

For more information, please contact researchrepository@mail.wvu.edu. 


\title{
Fluoride Thin Films: from Exchange Bias to Multferroicity
}

\author{
by Trent A. Johnson
}

Dissertation submitted

to the Eberly College of Arts and Sciences at West Virginia University

in partial fulfillment of the requirements for the degree of

Doctor of Philosopy in

Physics

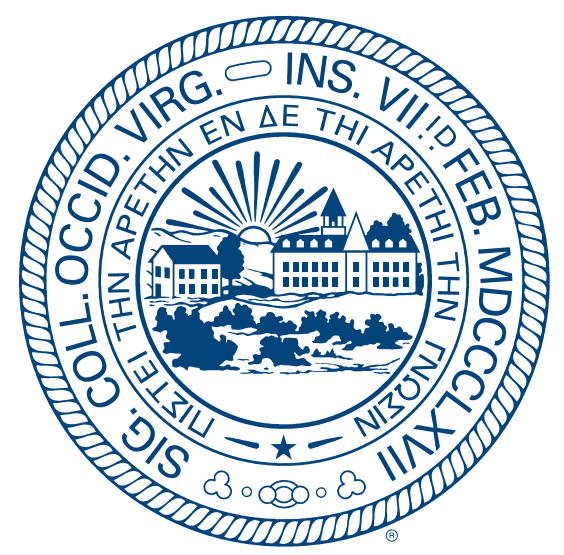

David Lederman, Ph.D., Chair

Pavel Borisov, Ph.D.

Edward Flagg, Ph.D.

Terry Gullion, Ph.D.

Tudor Stanescu, Ph.D.

Department of Physics and Astronomy

Morgantown, West Virginia

2015

Keywords:

Exchange Bias, Molecular Beam Epitaxy, Multiferroics Copyright 2015 Trent Johnson 


\title{
Abstract \\ Fluoride Thin Films: from Exchange Bias to Multiferroicity
}

\author{
Trent A. Johnson
}

This dissertation concerns research into the growth and characterization fluoride thin films by molecular beam epitaxy. After a discussion of relevant background material and experimental procedures in the first two chapters, we study exchange bias in magnetic multilayers incorporating the uniaxial antiferromagnet $\mathrm{FeF}_{2}$, grown to varying thicknesses, sandwiched between ferromagnetic Co layers with fixed thicknesses of 5 and $20 \mathrm{~nm}$. Several bilayers with only the $20 \mathrm{~nm}$ thick Co layer were grown for comparative study. The samples were grown on $\mathrm{Al}_{2} \mathrm{O}_{3}$ (1120) substrates at room temperature. In-situ RHEED and x-ray diffraction indicated the films were polycrystalline. The films were determined to have low surface and interlayer roughness, as determined by AFM and x-ray reflectivity. After field-cooling to below the Néel temperature of $\mathrm{FeF}_{2}$ in a magnetic field of $1 \mathrm{kOe}$, magnetic hysteresis loops were measured as a function of temperature. We found that both layers had a negative exchange bias, with the exchange bias of the thinner layer larger than that of the thicker layer. In addition, the coercivity below the blocking temperature $T_{B}$ of the thinner layer was significantly larger than that of the thick layer, even though the coercivity of the two layers was the same for $T>T_{B}$. The exchange bias effect, manifested by a shift in these hysteresis loops, showed a strong dependence on the thickness of the antiferromagnet. Anisotropic magnetoresistance measurements provided additional insight into the magnetization reversal mechanism within the ferromagnets. The thickness dependent exchange anisotropy of trilayer and bilayer samples is explained by adapting a random field model to the antiferromagnet/ferromagnet interface.

Finally, We investigate the temperature dependent growth, as well as the magnetic and ferroelectric properties of thin films of the multiferroic compounds $\mathrm{Ba} \mathrm{F}_{4}$, where $M=\mathrm{Fe}, \mathrm{Co}$, Ni. The films were grown to thicknesses of 50 or $100 \mathrm{~nm}$ on single crystal $\mathrm{Al}_{2} \mathrm{O}_{3}$ (0001) substrates. X-ray diffraction showed that this family of films grew epitaxially in the (010) orientation, but were twinned in the plane, with three domain orientations rotated by $120^{\circ}$ relative to one another. Measurements of the remanent hysteresis via interdigitated electrodes showed that the compounds $M=\mathrm{Co}$, and $\mathrm{Ni}$ were ferroelectric, but no switching behavior was observed in the Fe system at electric fields up to $400 \mathrm{kV} / \mathrm{cm}$. Measurements of the field-cooled and zero-field-cooled magnetic moment confirmed low temperature antiferromagnetic behavior, and found new weak ferromagnetic phases induced by strain. 


\section{Contents}

List of Figures . . . . . . . . . . . . . . . . . . viii

List of Tables ....................... . . . ix

1 Theoretical Background 1

1.1 Magnetic Order . . . . . . . . . . . . . . . . 1

1.1.1 Ferromagnetism and Antiferromagnetism . . . . . . . . . . 1

1.1.2 Exchange Interaction . . . . . . . . . . . . . . . 4

1.1.3 Dzialoshinskii-Moriya Interaction . . . . . . . . . . . 6

1.1.4 Exchange Bias . . . . . . . . . . . . . . . 7

1.2 Multiferroics . . . . . . . . . . . . . . . . . 11

1.2.1 Ferroelectricity . . . . . . . . . . . . . . 12

1.2.2 Magneto-Electric Coupling . . . . . . . . . . . . . . 13

1.3 Magnetoresistance . . . . . . . . . . . . . . . . . . . 14

1.3.1 Spin-dependent Scattering . . . . . . . . . . . . . 14

1.3.2 Anisotropic Magnetoresistance . . . . . . . . . . . . . . . 16

1.3.3 Tunneling and Giant Magnetoresistance . . . . . . . . . . . 18

2 Experimental Techniques $\quad 22$

2.1 Thin Film Growth . . . . . . . . . . . . . . . . . 22

2.1.1 Molecular Beam Epitaxy . . . . . . . . . . . . 22 
2.1.2 Electron Beam Evaporation . . . . . . . . . . . . . . 24

2.1.3 DC Magnetron Sputtering . . . . . . . . . . . . . . 25

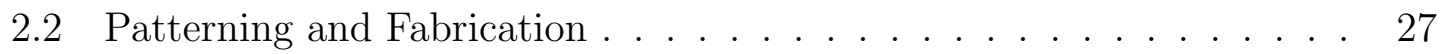

2.2 .1 Photolithography . . . . . . . . . . . . . . . 27

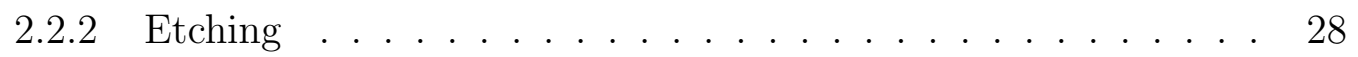

2.2 .3 Wire Bonding . . . . . . . . . . . . . . . . . . 30

2.3 Characterization Techniques . . . . . . . . . . . . . . 31

2.3.1 Reflection High Energy Electron Diffraction . . . . . . . . . 31

2.3 .2 X-ray Diffraction . . . . . . . . . . . . . . . . 33

2.3 .3 X-ray Reflectometry . . . . . . . . . . . . . . . 37

2.3.4 Atomic Force Microscopy . . . . . . . . . . . . . . . . . 37

2.3.5 Piezo Force Microscopy . . . . . . . . . . . . . . . . . . . 39

2.3 .6 Magnetometry . . . . . . . . . . . . . . . . . . . 40

2.3 .7 Magnetoresistance . . . . . . . . . . . . . . . . 43

2.3.8 Ferroelectric Properties Testing . . . . . . . . . . . . . . 44

3 Exchange Bias in $\mathrm{Co} / \mathrm{FeF}_{2} / \mathrm{Co}$ Trilayers 48

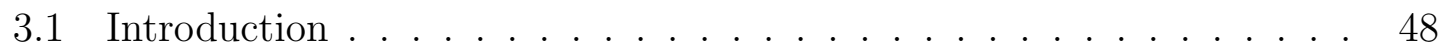

3.2 Experimental Procedures . . . . . . . . . . . . . . . . . . 49

3.2 .1 Growth . . . . . . . . . . . . . . . . . 49

3.2 .2 Structural Characterization . . . . . . . . . . . . 50

3.2 .3 Magnetic Characterization . . . . . . . . . . . . . 53

3.2 .4 Electrical Characterization . . . . . . . . . . . . . . 54

3.3 Results and Discussion . . . . . . . . . . . . . . . 55

4 Multiferroic ${\mathrm{Ba} M \mathrm{~F}_{4}}_{49}$ 
4.1 Introduction . . . . . . . . . . . . . . . 79

4.2 Experimental Procedures . . . . . . . . . . . . . . . . 81

4.2 .1 Growth ...................... 81

4.3 Results and Discussion . . . . . . . . . . . . . . . . 85

4.3.1 Structure .................... 85

4.3.2 Magnetic Characterization . . . . . . . . . . . . . 92

4.3.3 Ferroelectric Characterization . . . . . . . . . . . . . . 99

4.4 Summary . . . . . . . . . . . . . . . . . 107

$\begin{array}{ll}\text { Bibliography } & 116\end{array}$ 


\section{List of Figures}

1.1 Types of magnetic ordering . . . . . . . . . . . . . . 2

1.2 Magnetic hysteresis loop ................... 4

1.3 Hysteresis loop of Co-CoO nanoparticles . . . . . . . . . . . . . . 8

1.4 Phenomenological picture of exchange bias . . . . . . . . . . . . 9

1.5 Diagram of the Meiklejohn Bean model . . . . . . . . . . . . . 11

1.6 Exchange split density of states in transition metals . . . . . . . . . 16

1.7 Two current model of conduction in ferromagnets . . . . . . . . . . . 17

1.8 Anisotropic magnetoresistance . . . . . . . . . . . . . . . . . . . . 18

1.9 Two spin channel model of GMR . . . . . . . . . . . . . . . . . 20

2.1 Schematic of the fluoride/metals MBE system . . . . . . . . . . 23

2.2 Diagram of an e-beam evaporator . . . . . . . . . . . . 25

2.3 Depiction of DC magnetron sputtering . . . . . . . . . . . . 27

2.4 Stages of the photolithography process . . . . . . . . . . . . . 29

2.5 Schematic of the ultrasonic bonding process . . . . . . . . . . 31

2.6 Schematic of a RHEED system . . . . . . . . . . . . . . . 33

2.7 Scattering geometry in XRD . . . . . . . . . . . . . . 35

2.8 Four circle x-ray diffractometer . . . . . . . . . . . . . . 36

2.9 Diagram of reciprocal space in XRD . . . . . . . . . . . . . 36 
2.10 In-phase and out-of-phase piezoresponse $\ldots \ldots \ldots \ldots$

2.11 Diagram of a vibrating sample magnetometer . . . . . . . . . . 42

2.12 Six contact Hall bar . . . . . . . . . . . . . . . . . . . 44

2.13 Circuits for ferroelectric testing $\ldots \ldots \ldots \ldots \ldots \ldots$

2.14 Voltage vs. time characteristic for remanent hysteresis $\ldots \ldots \ldots 47$

3.1 RHEED image of polycrystalline trilayer . . . . . . . . . . 51

$3.2 \quad$ AFM image of $t_{A F}=3 \mathrm{~nm}$ sample $\ldots \ldots \ldots \ldots \ldots \ldots$

3.3 SLD profile and first derivative . . . . . . . . . . . . . 52

3.4 SLD profile of all trilayers $\ldots \ldots \ldots \ldots \ldots \ldots$

3.5 Magnetic hysteresis loop below and above $T_{B} \ldots \ldots \ldots \ldots 7$

3.6 Coercivity vs. temperature . . . . . . . . . . . . . . 58

3.7 Exchange field vs. temperature . . . . . . . . . . . . . . . 59

3.8 Interfacial exchange energy vs. temperature. . . . . . . . . . . 60

3.9 TRM vs. temperature for trilayers and bilayers $\ldots \ldots \ldots \ldots \ldots$

3.10 Blocking temperatures $\ldots \ldots \ldots \ldots \ldots \ldots$

3.11 Coercivity vs. AF thickness _. . . . . . . . . . . . . 65

3.12 Hysteresis of $t_{A F}=3 \mathrm{~nm}$ sample bilayer $\ldots \ldots \ldots \ldots 6$

3.13 Hysteresis of trilayer and bilayer . . . . . . . . . . . . 67

3.14 Normalized magnetoresistance at $14 \mathrm{~K} \ldots \ldots \ldots$. . . . . . 68

3.15 Low temperature coercivity vs. AF thickness . . . . . . . . . . . . 69

3.16 Hysteresis loop with AMR coercivity . . . . . . . . . . . . . . 70

3.17 Hysteresis loop under small negative cooling field . . . . . . . . . 71

3.18 Interface exchange energy vs. AF thickness at $\mathrm{T}=5 \mathrm{~K} \ldots \ldots . .72$

3.19 Bubble shaped AF domain in random field model . . . . . . . . 75

3.20 Domain walls vs. AF thickness _ . . . . . . . . . . . 76 
4.1 Unit cell of $\mathrm{BaMF}_{4} \ldots \ldots \ldots \ldots$. . . . . . . . . . 81

4.2 Polarized states of the $\mathrm{BaMF}_{4}$ structure $\ldots \ldots \ldots \ldots$. . . . 82

4.3 Diagram of the spin configuration in $\mathrm{BaMF}_{4} \ldots \ldots \ldots$. . . . 83

4.4 Two configurations of electrodes . . . . . . . . . . . . . . 84

4.5 XRD of $\mathrm{BaCoF}_{4}$ grown on $\mathrm{Pt}(111) \ldots \ldots \ldots \ldots$

4.6 AFM image of $\mathrm{BaCoF}_{4}$ film $\ldots \ldots \ldots \ldots \ldots$

4.7 RHEED image of $\mathrm{BaCoF}_{4}$ film . . . . . . . . . . . . . . 88

4.8 XRD of $\mathrm{BaFeF}_{4}$ grown on $\mathrm{Al}_{2} \mathrm{O}_{3}(0001) \ldots \ldots \ldots \ldots \ldots$

4.9 XRD of $\mathrm{BaCoF}_{4}$ grown on $\mathrm{Al}_{2} \mathrm{O}_{3}(0001) \ldots \ldots \ldots$

$4.10 \mathrm{XRD}$ of $\mathrm{BaNiF}_{4}$ grown on $\mathrm{Al}_{2} \mathrm{O}_{3}(0001) \ldots \ldots \ldots \ldots$

$4.11 \Phi$-scan of $\mathrm{BaCoF}_{4}$ grown on $\mathrm{Al}_{2} \mathrm{O}_{3} \ldots \ldots \ldots \ldots$

4.12 Orientation of $\mathrm{Ba} \mathrm{F}_{4}$ with respect to $\mathrm{Al}_{2} \mathrm{O}_{3}(0001)$ surface $\ldots . .93$

4.13 Rocking curves of $\mathrm{Ba} \mathrm{FF}_{4}$ films . . . . . . . . . . . . . . 94

$4.14 \mathrm{FC}$ and $\mathrm{ZFC}$ curves for $\mathrm{BaFeF}_{4} \ldots \ldots \ldots \ldots \ldots$

4.15 Magnetic hysteresis on $\mathrm{BaFeF}_{4} \ldots \ldots \ldots \ldots \ldots$. . . . . 96

$4.16 \mathrm{FC}$ and $\mathrm{ZFC}$ curves for $\mathrm{BaCoF}_{4} \ldots \ldots \ldots \ldots$

$4.17 \mathrm{TRM}$ of $\mathrm{BaCoF}_{4} \ldots \ldots \ldots \ldots \ldots \ldots \ldots$

4.18 Magnetic hysteresis on $\mathrm{BaCoF}_{4} \ldots \ldots \ldots \ldots$

$4.19 \mathrm{FC}$ and $\mathrm{ZFC}$ curves for $\mathrm{BaNiF}_{4} \ldots \ldots \ldots \ldots$

4.20 Magnetic hysteresis on $\mathrm{BaNiF}_{4} \ldots \ldots \ldots \ldots$

4.21 Photograph of interdigitated electrodes . . . . . . . . . . . . . 103

4.22 Averaged remanence of $\mathrm{BaCoF}_{4} \ldots \ldots \ldots \ldots$

4.23 Averaged remanence of $\mathrm{BaCoF}_{4} \ldots \ldots \ldots \ldots$

4.24 Averaged remanence of $\mathrm{BaNiF}_{4} \ldots \ldots \ldots \ldots \ldots$

4.25 Averaged remanence of $\mathrm{BaFeF}_{4} \ldots \ldots \ldots \ldots$ 


\section{List of Tables}

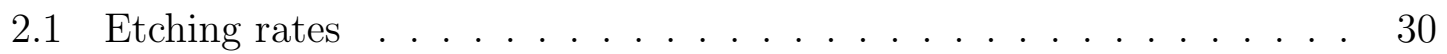

2.2 Etching parameters . . . . . . . . . . . . . . . . . . 30

3.1 Thickness and roughness parameters $\ldots \ldots \ldots \ldots \ldots$

3.2 Ratios of bottom vs. top Co layer magnetic moments . . . . . . . . 61

4.1 Atomic densities of di-fluorides . . . . . . . . . . . . . . . 85

4.2 Lattice parameters for $\mathrm{Ba} M \mathrm{~F}_{4}$ films . . . . . . . . . . . . . . 92

4.3 Lattice parameters for $\mathrm{Ba} M \mathrm{~F}_{4}$ bulk $\ldots \ldots \ldots \ldots \ldots$

4.4 Calculated energies of magnetic transitions in $\mathrm{BaCoF}_{4} \ldots \ldots \ldots 9$ 


\section{Chapter 1}

\section{Theoretical Background}

\subsection{Magnetic Order}

\subsubsection{Ferromagnetism and Antiferromagnetism}

All materials show a magnetic response to the application of an external magnetic field, but the nature and magnitude of those responses varies from material to material in accordance with the electronic structure of each. Take for example, the physically simple but heuristically useful model of a free, charged particle in a magnetic field. The particle will undergo cyclotron motion in the plane perpendicular to the applied field, resulting in an induced magnetic moment directed opposite to the orientation of the field. For the less trivial case of electrons bound in atomic orbitals, the Lorentz force perturbs the orbits in such a way as to produce the same type of effect, i.e. a negative induced magnetic moment, irrespective of the signs of the orbital or spin degrees of freedom. This response, known as diamagnetism, is present in all materials, but is generally weak, and not the dominant contributor to the net magnetization in many systems. 
(a)

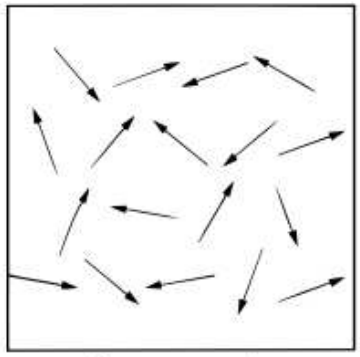

Paramagnetic

(c)

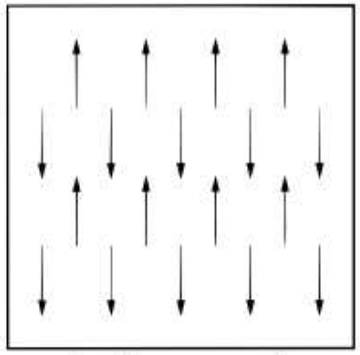

Antiferromagnetic (b)

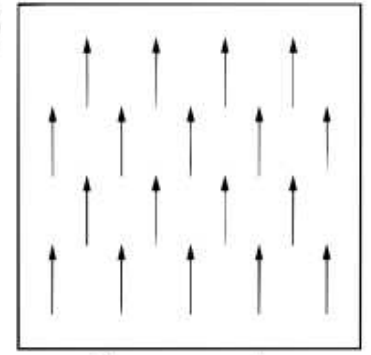

Ferromagnetic

(d)

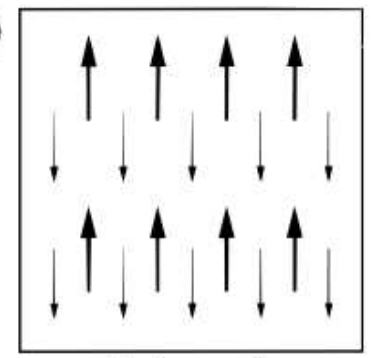

Ferrimagnetic

Figure 1.1: Types of magnetic ordering in materials reproduced from reference [1].

More interesting cases arise when constituent parts of a system have intrinsic magnetic moments $\boldsymbol{\mu}$, which preferentially align with an applied magnetic field $\mathbf{B}$ according to the energy

$$
\mathscr{E}=-\boldsymbol{\mu} \cdot \mathrm{B}
$$

yielding a net induced magnetization in the same direction as the field. This behavior, called paramagnetism, arises due to the presence of atoms with partially filled atomic orbitals in which the orbital and spin angular momenta of all the electrons combine to a non-zero sum. In the absence of an applied magnetic field the moments of a paramagnet will tend to be randomized by thermal interactions as depicted in figure $1.1(\mathrm{a})$.

Of greater interest for the present work are those subset of such materials in which short range interactions between intrinsic moments give rise to collective or- 
dering that can persist even in the absence of external magnetic fields. When these interactions favor parallel alignment of magnetic moments, as shown in figure 1.1(b), they produce a spontaneous net magnetization. This phenomenon is known as ferromagnetism (FM). When the anti-parallel arrangement of neighboring moments is favored, the material has no net magnetization, but still has a high degree of magnetic order. This is called antiferromagnetism (AF), and is illustrated in figure 1.1(c). As thermal energy tends to create disorder, both FM and AF ordering are temperature dependent, with ordering completely destroyed above a critical temperature. For FMs this temperature is called the Curie temperature $\left(T_{C}\right)$, and for AFs it is called the Néel temperature $\left(T_{N}\right)$. Closely related to the AF system is ferrimagnetism, which is a case in which the microstructure of the material is AF in nature, but due to an imbalance between the magnetic moments of the two anti-parallel sets of spins, there is a net magnetization.

The magnetic behavior of a FM can be well characterized by its hysteresis loop, which encapsulates not only the magnetization induced in response to a magnetic field, but also its "memory" of past magnetic states. A hysteresis loop is created by measuring magnetization as a function of magnetic field with the field first set to some high value (high enough to drive the magnetization to saturation $M_{s}$ ), then sweeping the field slowly to the same value in the opposite direction, and then back again to the starting field. As the magnetic field is decreased towards zero in the first leg of the loop, the magnetization generally decreases a little, leveling out at a remanent magnetization $M_{r}$ in zero field. This is because most of the time magnetic materials have preferred magnetic orientations governed by symmetries within the crystal structure. In zero applied field, the magnetization will tend to revert to one of these "easy axes." Therefore, $M_{r}$ compared to $M_{s}$ is an indication of the strength 
of this anisotropy. As the field is driven to negative values the magnetization will undergo an often rapid reversal. The field required to demagnetize the material is called the coercive field $H_{c}$. As the field continues to become more negative the magnetization eventually saturates in the negative direction, and the process repeats itself in inverted fashion in the second half of the loop.

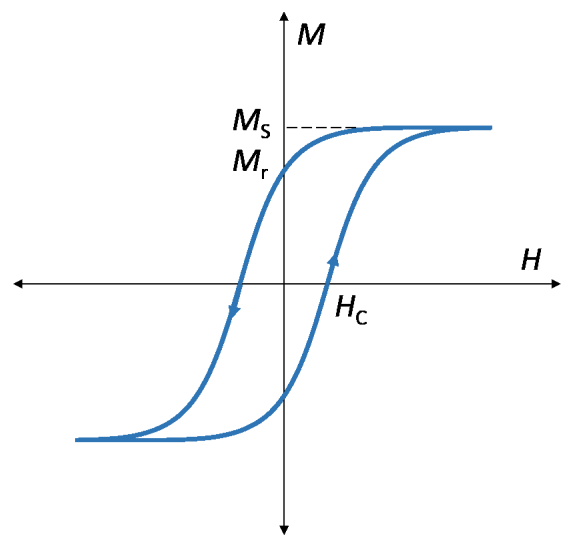

Figure 1.2: Magnetic hysteresis loop showing saturation magnetization $M_{s}$, remanent magnetization $M_{r}$, and coercive field $H_{c}$.

\subsubsection{Exchange Interaction}

The specific interactions between magnetic dipoles that give rise to $\mathrm{FM}$ and $\mathrm{AF}$ ordering are called exchange interactions. These interactions have no counterpart in macroscopic, classical magnetism, and must accordingly be treated quantum mechanically. Consider as a simple example, two electrons bound to different ions with spatial wave functions $\psi_{1}\left(\mathbf{r}_{1}\right)$ and $\psi_{2}\left(\mathbf{r}_{2}\right)$. Since these electrons are identical fermions, their total wavefunction must be antisymmetric under particle exchange. Therefore we could have a wavefunction $\Psi_{S}$, combining a spatially symmetric wavefunction with an antisymmetric, i.e. antiparallel spin configuration $\chi_{A}$, or wavefunction $\Psi_{A}$ with a spatially antisymmetric part and a symmetric, i.e. parallel spin configuration 
$\chi_{S}:$

$$
\begin{aligned}
\Psi_{S} & =\frac{1}{\sqrt{2}}\left[\psi_{1}\left(\mathbf{r}_{1}\right) \psi_{2}\left(\mathbf{r}_{2}\right)+\psi_{1}\left(\mathbf{r}_{2}\right) \psi_{2}\left(\mathbf{r}_{1}\right)\right] \chi_{A} \\
\Psi_{A} & =\frac{1}{\sqrt{2}}\left[\psi_{1}\left(\mathbf{r}_{1}\right) \psi_{2}\left(\mathbf{r}_{2}\right)-\psi_{1}\left(\mathbf{r}_{2}\right) \psi_{2}\left(\mathbf{r}_{1}\right)\right] \chi_{S} .
\end{aligned}
$$

It is clear from the above equations that if there is no overlap between $\psi_{1}(\mathbf{r})$ and $\psi_{2}(\mathbf{r})$ then $\left|\Psi_{S}\left(\mathbf{r}_{1}, \mathbf{r}_{2}\right)\right|^{2}=\left|\Psi_{A}\left(\mathbf{r}_{1}, \mathbf{r}_{2}\right)\right|^{2}$ and the expectation value of $\mathbf{r}_{1}-\mathbf{r}_{2}$ would be the same for the spatially symmetric and antisymmetric combinations. Hence, the Coulomb energy

$$
\mathscr{E}=\frac{1}{4 \pi \epsilon_{0}} \frac{e^{2}}{\left|\mathbf{r}_{1}-\mathbf{r}_{2}\right|},
$$

would be degenerate for $\Psi_{S}$ and $\Psi_{A}$. However, if the wavefunctions have a spatial overlap, then this degeneracy would be lifted and the Coulomb energies of the two combinations would have a difference of

$$
\Delta \mathscr{E}=J=2 \frac{1}{4 \pi \epsilon_{0}} \int \psi_{1}^{*}\left(\mathbf{r}_{1}\right) \psi_{2}^{*}\left(\mathbf{r}_{2}\right) \frac{e^{2}}{\left|\mathbf{r}_{1}-\mathbf{r}_{2}\right|} \psi_{1}\left(\mathbf{r}_{2}\right) \psi_{2}\left(\mathbf{r}_{1}\right) d \mathbf{r}_{1} d \mathbf{r}_{2}
$$

Since these energies are coupled to parallel and antiparallel configurations of the electron spins, the Coulomb energy can be parameterized and expanded as a power series in $\mathbf{s}_{1} \cdot \mathbf{s}_{2}$ where $\mathbf{s}_{1}$ and $\mathbf{s}_{2}$ are the spin operators associated with $\psi_{1}\left(\mathbf{r}_{1}\right)$ and $\psi_{2}\left(\mathbf{r}_{2}\right)$ respectively [2]. Customarily this expansion is taken only to first order as an effective spin-dependent Coulomb Hamiltonian of the form

$$
\mathscr{H}_{\text {Coulomb }}=-J \mathbf{s}_{1} \cdot \mathbf{s}_{2},
$$


although higher order terms can sometimes be added [3]. A more general treatment for multi-electron atoms (see ref [2]) yields the Heisenberg Hamiltonian

$$
\mathscr{H}_{\text {Heisenberg }}=-2 \sum_{i>j} J_{i j} \mathbf{S}_{i} \cdot \mathbf{S}_{j},
$$

where $\mathbf{S}_{i}$ and $\mathbf{S}_{j}$ are the total spins of ions $i$ and $j$, and $J_{i j}$ is known as the exchange integral or exchange constant for the given pair of spins. Being dependent upon the overlap between electron wavefunctions, both the sign and magnitude of $J$ are highly sensitive to the spacing between ions in a crystal. Clearly, when $J$ is positive a parallel orientation of the spins is favored and the interaction is FM, while negative $J$ produces an AF interaction.

The above description of the exchange interaction as a phenomenon arising due to overlap of electrons on neighboring ions is known as direct exchange. However, in many cases (e.g. the $4 f$ electrons in the rare earths) the orbital overlap is too small to account for observed magnetic ordering. These instances can be explained by mechanisms in which two magnetic ions interact with one another by coupling to intermediary spins, such as conduction electrons in metals or spin neutral atoms in ionic solids [4]. These types of interactions, of which there are several varieties, are collectively called indirect exchange interactions.

\subsubsection{Dzialoshinskii-Moriya Interaction}

In some AFs there appears a small, spontaneous magnetic moment which cannot be explained by ferromagnetic impurities or domain walls. That is, this weak

ferromagnetism is an inherent byproduct of the AF system. Dzialoshinskii [5] first proposed a phenomenological model to explain such behavior in $\alpha-\mathrm{Fe}_{2} \mathrm{O}_{3}$ with an 
antisymmetric exchange interaction of the form:

$$
\mathscr{H}_{D M}=\mathbf{D} \cdot\left(\mathbf{S}_{1} \times \mathbf{S}_{2}\right),
$$

where the vector $\mathbf{D}$ is partially determined by symmetry, and hence not allowed for crystal structures.

By itself this energy would clearly tend to orient the spins at $90^{\circ}$ to one another in the plane perpendicular to $\mathbf{D}$. Acting in conjunction with a symmetric exchange of the form of equation 1.6, this produces a canting of the spins away from the principal direction of AF ordering. Moriya [6] described this theoretically by extending the theory of superexchange developed by Anderson [7] to include the mixing of spin-up and spin-down states produced by spin-orbit coupling. He showed that

$$
D \approx \frac{\Delta g}{g} J_{\text {super }},
$$

where $J_{\text {super }}$ is the strength of the superexchange interaction, and $\Delta g$ is the deviation of the electron's $g$-factor away from 2 . Since $\Delta g / g \ll 1$ for $3 d$ ions, the superexchange dominates and the canting produced is $3^{\circ}$ at most [4].

\subsubsection{Exchange Bias}

Usually, the hysteresis loop of a FM is centered around zero field. However, in systems where the FM (or ferrimagnet) has an interface with an AF material, the loop can undergo a horizontal shift, provided the FM is magnetized as the system passes through the AF critical temperature $T_{N}$. This phenomenon is known as exchange bias (EB), and is the result of anisotropic exchange interactions at the interface. The shift is quantified by the exchange field $\mu_{0} H_{E B}$, which is usually negative with 
respect to the magnetization direction during cooling, but can be positive [8]. The effect was first discovered by Meiklejohn and Bean in Co nanoparticles surrounded with a shell of $\mathrm{CoO}[9]$. Since that time, EB has been observed other systems, such as thin films, and has found widespread application in magnetic memory [10] and other magnetic devices.

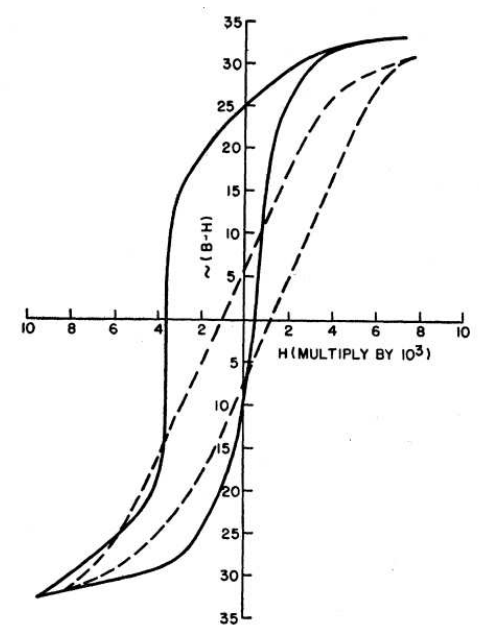

Figure 1.3: Hysteresis loops of $\mathrm{Co}-\mathrm{CoO}$ nanoparticles at $77 \mathrm{~K}$ take by Meiklejohn and Bean [9]. The dashed loop is without field cooling, and the solid loop is the exchange biased one, cooled in a saturating field.

A qualitative picture of the microscopic origins of $\mathrm{EB}$ can be gleaned from figure 1.4. The magnetic moments at the interface always interact via exchange interactions, but the unidirectional anisotropy that gives rise to $\mathrm{EB}$ is governed by the thermodynamic conditions. If a large enough magnetic field is applied in the temperature range $T_{N}<T<T_{C}$, the FM will become magnetized in the direction of the applied field while the AF remains in a paramagnetic state. Since the interfacial exchange energies are random in sign at different lattice sites, they make no net contribution to the energy of the system as a whole. Accordingly EB is absent and the FM reverses independently of the presence of the AF. If the system is cooled to below $T_{N}$, long range order will develop in the $\mathrm{AF}$, but in a non-random way. The AF will 
order in such a way as to minimize the interface exchange energy by allowing the first monolayer of spins to align parallel (anti-parallel) to the their neighbors in the FM according to whether the interface coupling is FM or AF. Given a high enough anisotropy, the AF spins, once ordered, become frozen in this configuration. In order to reverse, the FM spins must overcome the torque exerted by the interfacial coupling in the direction of the cooling configuration. The same torque aids in reversing the spins again to their original position, effectively adding an internal bias to the system [11]. The EB persists below the blocking temperature $\left(T_{B}\right)$, which is usually near $T_{N}$, although in the case of very thin FM layers it can be significantly below the Néel temperature [12].

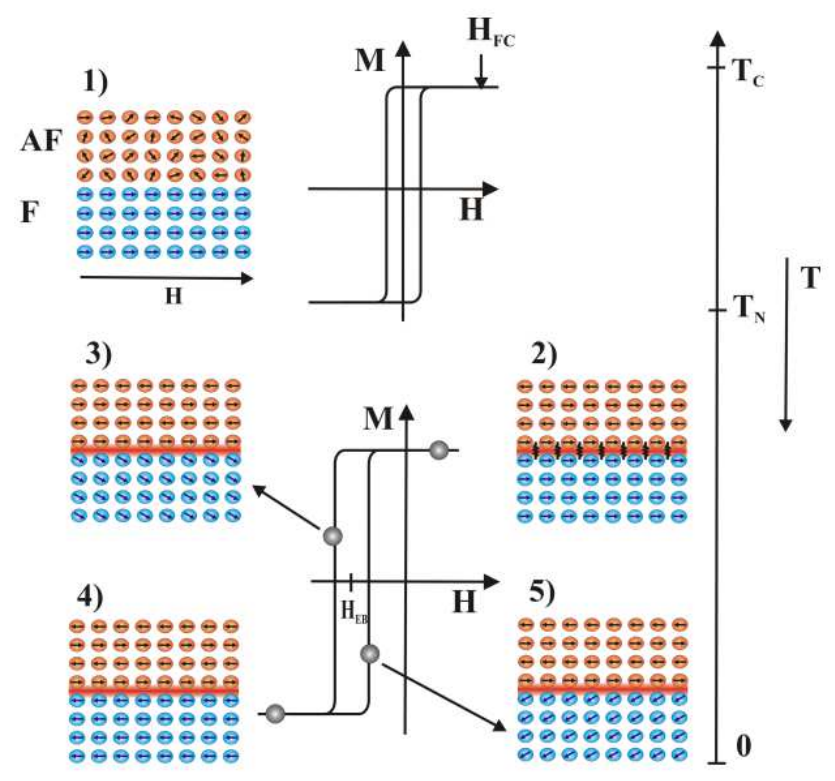

Figure 1.4: A phenomenological picture of exchange bias. 1) For $T_{N}<T<T_{C}$ the FM is magnetized while the AF is paramagnetic. The corresponding hysteresis loop is unbiased. 2) After field cooling to below $T_{N}$ the AF spins have coupled to the FM spins at the interface. 3) The spin configuration during reversal; the AF spins exert a torque on the FM. 4) The spin configuration at negative saturation. 5) During the reversal back to the original magnetization, the interfacial torque aids in the reversal. Reproduced from reference [13]. 
The first theoretical model to describe EB quantitatively is due to Meiklejohn and Bean [14,15], based on a modified version of the Stoner-Wohlfarth model [16] of coherent magnetization rotation. In this treatment, the FM and AF layers are assumed to have collinear uniaxial anisotropy, and they are magnetically rigid, single domains that rotate coherently and independently as shown in figure 1.5. The energy per unit area of this system can be expressed as:

$$
\mathscr{E}=-\mu_{0} H M_{F M} t_{F M} \cos (\theta-\beta)+K_{A F} t_{A F} \sin ^{2}(\alpha)-J_{E B} \cos (\beta-\alpha),
$$

where $K_{F M}$ and $K_{A F}$ are the anisotropy constants, $t_{F M}$ and $t_{A F}$ are the thicknesses, and $\beta$ and $\alpha$ are the angles of magnetic rotation for FM and AF layers, respectively. The magnetization of the FM, $M_{F M}$ is assumed to be saturated in magnetic field $H$, while $J_{E B}$ is the interface exchange coupling per unit area. Minimization of equation 1.9 with respect to $\alpha$ and $\beta$ yields the relation [15] for the EB field:

$$
\mu_{0} H_{E B}=\frac{J_{E B}}{M_{F M} t_{F M}} .
$$

The prediction that $H_{E B}$ is inversely proportional to $t_{F M}$ has been widely studied [17], because it is a reflection of the interfacial origin of EB. For thick FM films the $1 / t_{F M}$ trend is widely observed, but it breaks down for very thin films in which the film is likely to be discontinuous. A major problem with applying Eq. 1.10 is that the interface coupling $J_{E B}$ is generally difficult to estimate for real interfaces as it can be complicated by a number of factors, such as the surface roughness, structure, and magnetic ordering. Estimates of $J_{E B}$ based upon completely smooth, completely uncompensated (only one AF sublattice present at the interface) surfaces routinely generate dramatic overestimations of the magnitude of $H_{E B}$ [11]. Additionally, nom- 


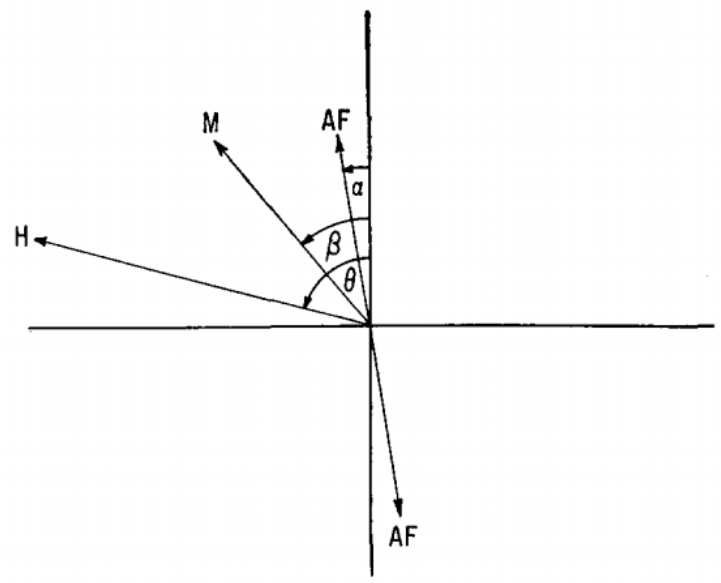

Figure 1.5: Diagram of the angles and vectors used in the Meiklejohn and Bean model of EB [15].

inally compensated (both sublattice magnetizations present at the interface) surfaces often exhibit EB, which should be zero under this simple picture. A host of models have been developed, which invoke domain walls parallel $[18,19]$ or perpendicular [20] to the interface, types of magnetic disorder [21,22], or other mechanisms to explain EB. While many of these models are partially successful in certain systems, the role of the various degrees of freedom (anisotropy, roughness, magnetic order during reversal, etc.) on the microscopic origin of EB is not yet well understood [11,23].

\subsection{Multiferroics}

Multiferroics are materials which simultaneously exhibit two or more types of ferroic (or anti-ferroic) order, e.g. ferroelectricity, ferromagnetism, or ferroelasticity. Accordingly, they have attracted much interest due to their potential applications in devices with novel or multiple functionalities. Of particular interest is that subset of multiferroics in which the ordering mechanisms do not occur independently, but rather are coupled. For example, one might produce a change in magnetization via 
an applied voltage.

\subsubsection{Ferroelectricity}

Of the 32 point groups into which crystal structures can be classified, 11 have a center of symmetry, which excludes a net dipole moment. Of the remaining 21 non-centrosymmetric groups, 20 are piezoelectrics, which is to say, the crystal lattice develops a net dipole moment when it is distorted under physical stress or vice versa [24]. A further subset of 10 groups have a polar symmetry, which gives rise to a spontaneous dipole moment in the unit cell. That moment is subject to change with temperature, and those materials are thus known as pyroelectrics. Those pyroelectrics whose sponateous polarization can be switched by the application of an electric field are said to be ferroelectric, by analogy with ferromagnets. Because they exhibit this interplay between physical strain, temperature, and electric field, ferroelectrics have a wide variety of applications in devices such as sensors, transducers, high permittivity capacitors, memory elements, etc. [25].

Similar to their ferromagnetic counterparts, ferroelectrics form polarized domains, and exhibit hysteresis in their polarization as a function of electric field. Therefore, they can be characterized by quantities like coercive field $E_{c}$, saturation polarization $P_{s}$, and remnanent polarization $P_{r}$. Likewise they undergo a phase transition at a critical temperature $T_{C}$ to an ordinary dielectric. The displacement of charges to form a dipole moment generates an inherent electro-static instability which can only exist because it is counteracted by short range repulsive forces arising from the exclusion principle. At higher temperatures these short range forces dominate and produce the unpolarized charge symmetric structure. 


\subsubsection{Magneto-Electric Coupling}

In the most generic form, the polarization and magnetization of materials could be expanded in the form:

$$
\begin{gathered}
P_{i}(\mathbf{E}, \mathbf{H})=P_{i}^{s}+\epsilon_{0} \epsilon_{i j} E_{j}+\alpha_{i j} H_{j}+\ldots \\
\mu_{0} M_{j}(\mathbf{E}, \mathbf{H})=\mu_{0} M_{j}^{s}+\mu_{0} \chi_{i j} H_{i}+\alpha_{i j} E_{i}+\ldots,
\end{gathered}
$$

to include the response of polarization to magnetic fields and magnetization to electric fields [26]. The tensor $\alpha_{i j}$ is the linear magneto-electric (ME) coupling, and $\epsilon_{i j}$ and $\chi_{i j}$ are the electric and magnetic susceptibilities, respectively. In order for such a term to exist the material must simultaneously break time reversal and spatial inversion symmetries [26]. Additionally, the size of the effect is limited by the relation

$$
\alpha_{i j}^{2}<\epsilon_{i i} \chi_{j j} .
$$

Therefore, while it is not strictly speaking necessary for a material to be multiferroic to exhibit a ME effect, they are the best candidates for applications, due to the high permittivity and permeability they possess [1].

While symmetry considerations impose necessary conditions for the existence of ME coupling, a detailed understanding of its origins must be derived from microscopic interactions. Magnetic materials generally have a preference for ordering along particular directions in a crystal lattice. This magnetocrystaline anisotropy arises because a magnetic ion experiences a local electrostatic field (ligand field) due to surrounding ions which has the same symmetry as the crystal lattice. The atomic orbitals preferentially align in such a fashion as to minimize their energy in the anisotropic field. Because of spin-orbit coupling, the magnetic moment of each such 
ion is coupled to the orientation of its orbitals. The application of an electric field often causes distortion of the crystal lattice, which in turn alters the magnitude or symmetry of the ligand field, changing the magnetic anisotropy. The distortion of the electronic wavefunctions in the presence of a field can also change the electron overlap, bond length, or bond angle, and hence the strength or direction of exchange interactions [26].

\subsection{Magnetoresistance}

It has been known since the middle of the nineteenth century that conductors exhibit a change in resistivity in the presence of an applied magnetic field [27]. This ordinary magneto-resistance is common to all conductors, but is usually a very small effect. In the past century and a half other forms of magnetically induced changes in electrical resistance have been discovered, of which several will be analyzed here.

\subsubsection{Spin-dependent Scattering}

While it is tempting and at times useful to think in terms of discrete orbitals, a fuller understanding of magnetic and electrical transport properties of real materials must take into account the band structure of electrons. In the FM transition metals, the $3 d$ electrons that are responsible for their magnetism form a conduction band along with more loosely bound $4 s$ electrons. Because the $d$ electrons are relatively tightly bound, they form a narrow band with a large density of states. Consequently, their effective mass

$$
m^{*}=\hbar^{2}\left[\frac{d^{2} \mathscr{E}}{d k^{2}}\right]^{-1}
$$


is large and they have a low mobility. Cutting across this band is an $s p$ hybrid band with small effective mass and high mobility. This picture neatly explains the properties of the transition metals, which have a relatively high resistance because of their high density of states at the Fermi level. Alternatively $\mathrm{Cu}$, which is highly conducting and non-magnetic because its $d$-band is full with the Fermi energy entirely within the high mobility $4 s$-band.

The exchange interaction lifts the spin-degeneracy of the $d$ electrons causing it to split into two sub-bands, one for majority $(+)$ and another for minority $(-)$ electrons, with different density of states at the Fermi energy. According to the Stoner criterion, if the density of states at the Fermi level times the exchange energy is larger than one the ground state will a polarization, i.e. two unequal spin populations and hence a net magnetic moment, i.e. FM prevails. Of the transition metals, only $\mathrm{Fe}, \mathrm{Co}$, and $\mathrm{Ni}$ meet this criterion and therefore display FM behavior [4]. For these metals, a decrease in resistivity occurs when the material transitions from the paramagnetic to FM phases, suggesting that electron spin plays a role in the scattering mechanism.

Mott [28] explained this phenomenon using a two current model of conduction in the exchange split sub-bands. He assumed that most of the conduction was due to the high mobility $s$ electrons and that the dominant contribution to resistance came from scattering between the $s$ and $d$-bands. The frequency of a given scattering event is proportional to the number of ways that event can occur. Since the $d$-band has a higher density of states at the Fermi level, there are more available states for an $s$ electron to scatter into than in the $s$-band. Well below the Curie temperature, where spin-flip scattering is negligible, the current can be split into two parallel spin channels with an equivalent resistance shown in figure 1.7. Because the $s$-band is spin-degenerate, the probabilities for majority and minority spin $s-s$ scattering 


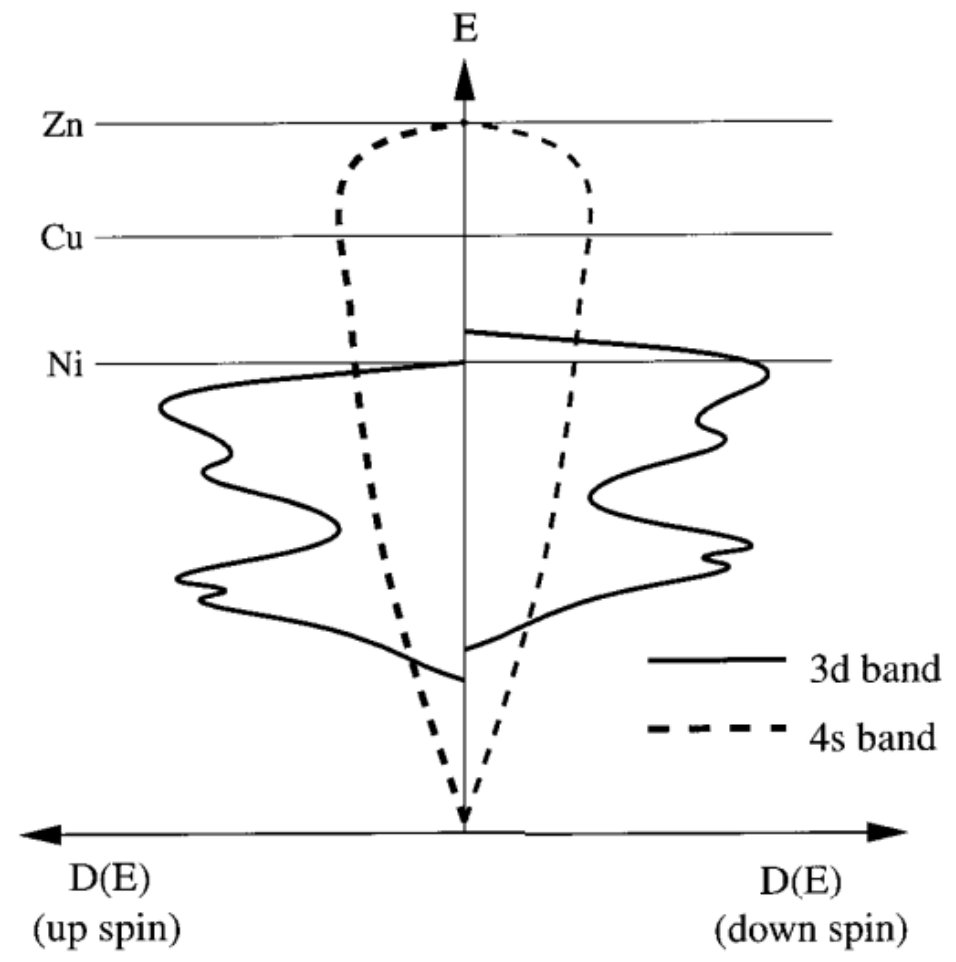

Figure 1.6: Density of states for $3 d$ and $4 s$ bands in the transition metals showing exchange splitting in the $d$-band as well as Fermi levels for $\mathrm{Ni}, \mathrm{Cu}$, and $\mathrm{Zn}$. Reproduced from $[1]$.

events are the same. However, with the exchange split $d$-band this is not the case. Mott showed that for the case of Ni the majority sub-band is completely full. Hence there are no states available to scatter into, and $R_{s+d+}$ is zero.

\subsubsection{Anisotropic Magnetoresistance}

In 1857, William Thompson [29] discovered that in FM materials the resistivity varies with the relative angle between the current density and the magnetization, a phenomenon now known as anisotropic magnetoresistance (AMR). For a single 


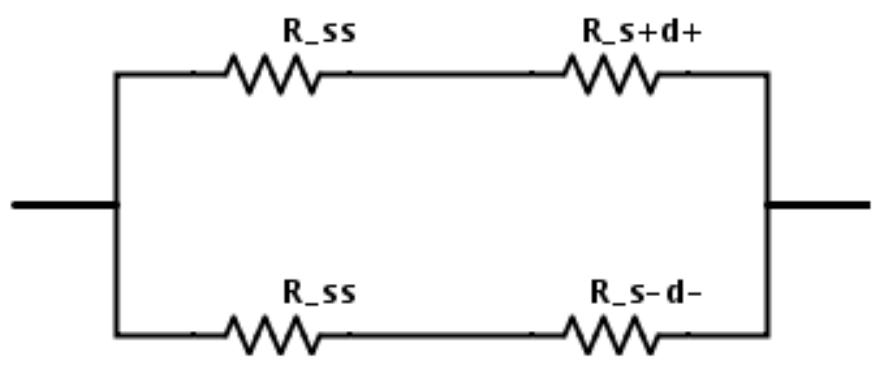

Figure 1.7: Equivalent circuit for two current conduction in a transition metal FM.

domain, thin film sample the resistivity takes the form:

$$
\rho(\theta)=\rho_{\perp} \sin ^{2} \theta+\rho_{\|} \cos ^{2} \theta,
$$

where $\theta$ is the angle between the magnetization and the current density, while $\rho_{\|}$ and $\rho_{\perp}$ are the resistivities parallel and perpendicular to the current, respectively. Generally $\rho_{\|}>\rho_{\perp}$, however this is not always the case, and the the angular dependence in real metals can be complicated by a host of factors including: domain boundaries, lattice defects, impurities, and sample geometry [30]. While this makes AMR difficult to analyze in specific materials, the general mechanism is understood to arise from the confluence of spin-orbit coupling and the exchange interaction.

A simple heuristic explanation of AMR is as follows. The electrons that give rise to an ion's magnetic moment have angular momenta $\mathbf{L}$ with spherically asymmetric orbitals, which are connected to the total moment by $\mathbf{L} \cdot \mathbf{S}$ coupling. Hence the asymmetric orbitals rotate with the magnetization. A conduction electron passing through the material effectively "sees" an electron cloud around each ion whose cross-sectional profile depends upon the orientation of the magnetic moment. This presents an intuitively appealing picture of why $\rho_{\|}$is usually greater than $\rho_{\perp}$. A hydrogen-like electron $d$-orbital viewed toward the plane of rotation appears to take 
up more space than when viewed from the side. Accordingly, electrons traveling along the spin axis are more likely to scatter (higher resistivity) than those traveling in the orbital plane (lower resistivity).

(a)

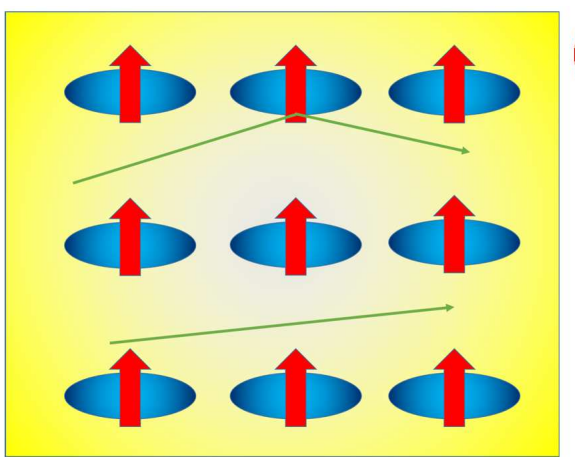

(b)

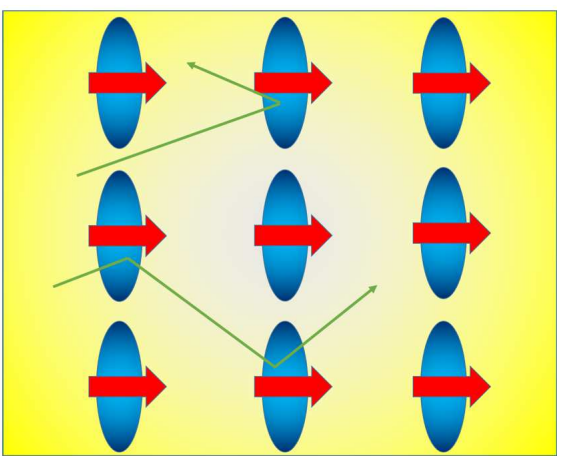

Figure 1.8: Depiction of anisotropic magnetoresistance for current (a) perpendicular and (b) parallel to the magnetization.

A more quantitative description of AMR in the transition metals was performed by Smit [31] and Berger [32], who treated the spin-orbit interaction

$$
\mathscr{H}_{\text {spin-orbit }}=\lambda \mathbf{L} \cdot \mathbf{S}=\lambda\left(L_{z} S_{z}+\frac{1}{2}\left(L_{+} S_{-}+L_{-} S_{+}\right)\right)
$$

as a small perturbation acting on tight binding $d$-orbitals. The scattering matrix elements of plane-wave $\psi_{s}=e^{i \mathbf{k} \cdot \mathbf{r}} s$-electrons with the perturbed $d$-wavefunctions are different for $k_{x}, k_{y}$ and $k_{z}$, and predict $\rho_{\|}>\rho_{\perp}$.

\subsubsection{Tunneling and Giant Magnetoresistance}

Giant magnetoresistance (GMR) and tunneling magnetoresistance (TMR) are two closely related phenomena which arise in multi-layered magnetic structures. GMR was first identified in superlattices of $\mathrm{Fe}$ and $\mathrm{Cr}$, where the FM Fe layers coupled antiferromagnetically to one another through the non-magnetic $\mathrm{Cr}$ layers 
via RKKY (Ruderman-Kittel-Kauya-Yosida) exchange [33]. This is a type of superexchange between inner $d$-electrons mediated by conduction electrons [4]. When the magnetizations of adjacent layers were brought into parallel alignment by an external magnetic field a substantial decrease in resistance through the multi-layer of about $50 \%$ was observed. Behavior of this nature and magnitude could not be accounted for by the anisotropic magnetoresistance of the materials involved, hence an entirely new phenomenon had been discovered. GMR in other structures can produce resistivity changes on the order of hundreds of percent. Consequently, GMR finds practical applications in devices, particularly in magnetic memory [34].

The origin of GMR can be neatly described by the two-current model of conduction in FMs described above. A simple model is presented in the figure, where we consider two identical magnetized layers with a current flowing transversely through them. The conduction electrons in the first layer are split into majority and minority spin populations defined by the orientation of the magnetization. The majority electrons have a lower resistance through the first layer than the minority electrons due to the higher number of vacant $d$-states in the minority sub-band. As long as the thickness of the non-magnetic layer is sufficiently small that the electrons can diffuse through it without undergoing spin-flip scattering, we can apply the two current model to both FM layers. If the magnetizations of the two layers are parallel then the resistance of each spin channel is unchanged and the second layer has the same resistance as the first, as shown in the equivalent circuit in figure 1.9. However, when the magneitizations are anti-parallel, the electrons in the majority spin channel become minority electrons in the second layer and vice versa. This means that the equivalent resistances are inverted as shown in the circuit of the figure. It can be straightforwardly shown that the equivalent resistance of the anti-parallel case is 
higher than that of the parallel one.
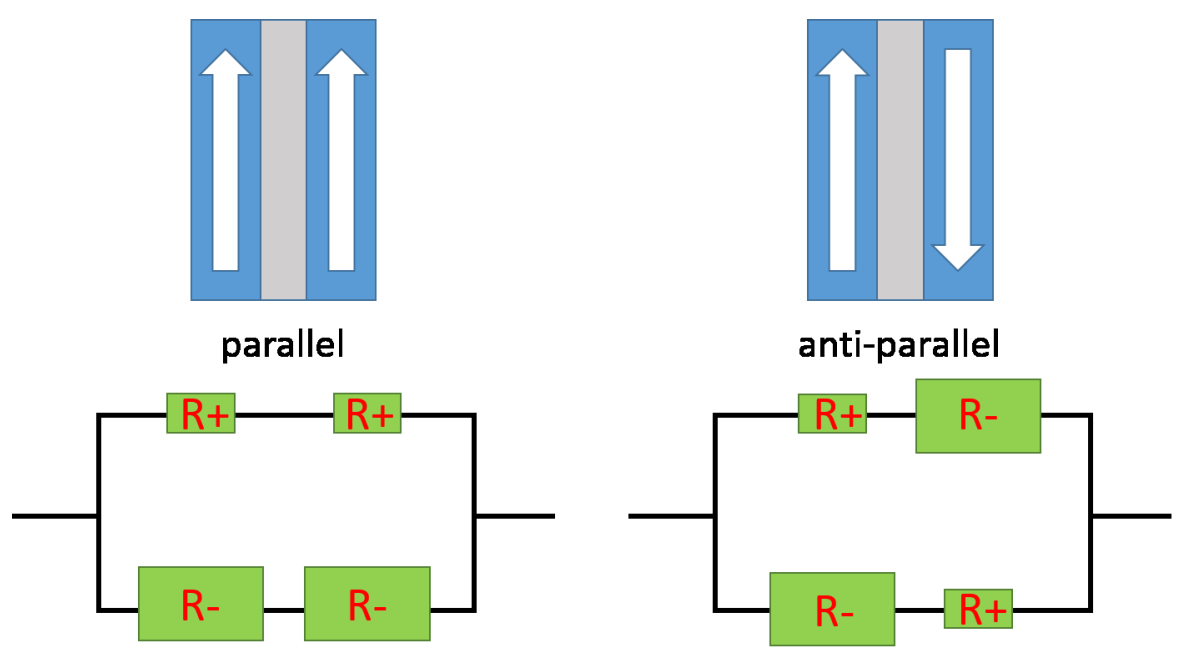

Figure 1.9: Ferromagnetic bilayers separated by a nonmagnetic spacer with parallel and anti-parallel magnetizations. The spin channels experience resistances $R_{+}$and $R_{-}$for majority and minority spins as in the corresponding equivalent circuits.

The structures that give rise to TMR (magnetic tunnel junctions) are similar to those that produce GMR, except that the non-magnetic conductor of the later case is replaced by an insulating tunneling barrier in the former. The physics governing the current flow through the entire structure is largely the same as that of GMR, with a change in resistance between parallel/anti-parallel magnetizations determined by the density of states in the FM layers. However, the tunneling barrier contributes an additional resistance, which may or may not have spin filtering properties of its own. If majority and minority spins have different transmission coefficients then the additional contribution of the tunneling barrier can therefore greatly increase the change in resistance through the structure. This can be done either by using a magnetic insulator such as EuS [35], or with epitaxial films of materials like $\mathrm{MgO}$ [36] that preserve the coherence of the tunneling wavefunctions. In either case, the effective height of the energy barrier is spin dependent, however, details concerning 
the effects of disorder on the tunnel properties are not well understood [37]. 


\section{Chapter 2}

\section{Experimental Techniques}

\subsection{Thin Film Growth}

\subsubsection{Molecular Beam Epitaxy}

Molecular Beam Epitaxy (MBE) is a technique used for the growth of high quality thin films. As the name suggest, a beam of elemental or molecular constituents impinges on the surface of a substrate where they can order themselves upon (epitaxy $=$ above) the substrate in accordance with the substrate's crystal structure. MBE systems operate in ultra high vacuum (typically with background pressures on the order of $10^{-10}$ Torr), thereby reducing the occlusion of contaminants during growth. Another benefit of the low pressure environment is that the mean free path of the particles in the beam

$$
L_{b}^{-1}=\sqrt{2} \pi n_{b} d_{b}^{2}+\pi n_{g} d_{b g}^{2} \sqrt{1+v_{g}^{2} / v_{b}^{2}}
$$


is on the order of several meters, much larger than the distance between the source and substrate. In equation $2.1 n_{b}, d_{b}$, and $v_{b}$ are respectively the concentration, diameter, and average velocity of the beam constituents. Corresponding terms with the subscript $g$ are the same parameters for the residual gas species in the chamber, and $d_{b g}=\left(d_{b}+d_{g}\right) / 2$ [38]. Therefore, the constituent particles only react at the surface, allowing for precise stoichiometric control from co-depositing sources. Also the relatively slow deposition rate allows the adsorbates to migrate and restructure themselves epitaxially on the deposition surface. These qualities make MBE an ideal method for materials research environments where high quality is required, but not high throughput. The vacuum environment also makes MBE compatible with a host of in-situ characterization methods, such as reflection high energy electron diffraction [39] (see below), low energy electron diffraction (LEED), and Auger electron spectroscopy (AES) [40].

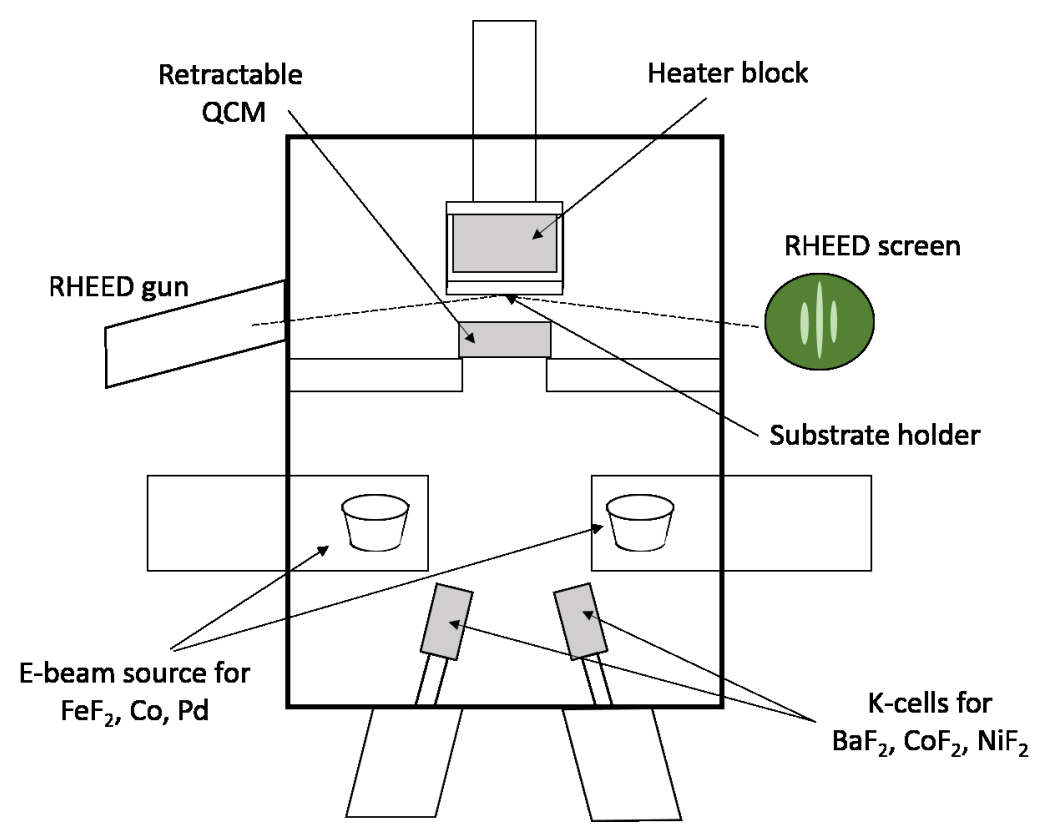

Figure 2.1: Schematic of the dedicated fluoride/metals MBE system used to grow the samples discussed in this work. 
The deposition source in MBE is typically from what is known as a Knudsen effusion cell or k-cell. A k-cell consists of essentially a highly thermo-tolerant, nonreactive crucible, which houses the source material. The exterior of the crucible is wound with a resistive heating filament, which radiatively heats the crucible and source material until material sublimates from source [41]. Those constituents that effuse through the top opening of the crucible travel ballistically, due to the low mean free path, forming the beam that gives MBE its name. The effusion flux can be controlled by adjusting the temperature via the filament current using feedback from an appropriately selected thermocouple in contact with the crucible. In turn, the flux can be monitored by a quartz crystal monitor (QCM), which operates on the principle that a quartz crystal resonator modulates its oscillation frequency in response to a change of mass [41], in this case due to accumulation of condensed material from the molecular beam. Often multiple k-cells are used in conjunction, either for simultaneous co-deposition or for the creation of multi-layered structures.

\subsubsection{Electron Beam Evaporation}

Electron beam evaporation is a thin film deposition process that can be utilized in an MBE system as described in the present work (see chapters 3 and 4), but can also be used as a stand-alone method as it does not require as low of an operational pressure and generally provides higher deposition rates than k-cells [42], and can be utilized with source materials with melting points that are too high for evaporation. The basic setup used in our system (e-vap CVS manufacured by MDC vacuum procucts, LLC) is as sketched in figure 2.2. A beam of electrons is emitted from a filament and accelerated by a high voltage $(5 \mathrm{kV})$. The beam is bent into an arc by permanent magnets and directed onto a crucible containing source material. The 
kinetic energy of the electrons is transferred to the source, heating it until it melts or sublimates, producing a vapor that condenses on the substrate. Secondary magnetic fields generated by coils in the housing raster the beam over the source to produce more uniform heating. The hearth surrounding the crucible is cooled by water flow, which is interlocked to the filament power supply.

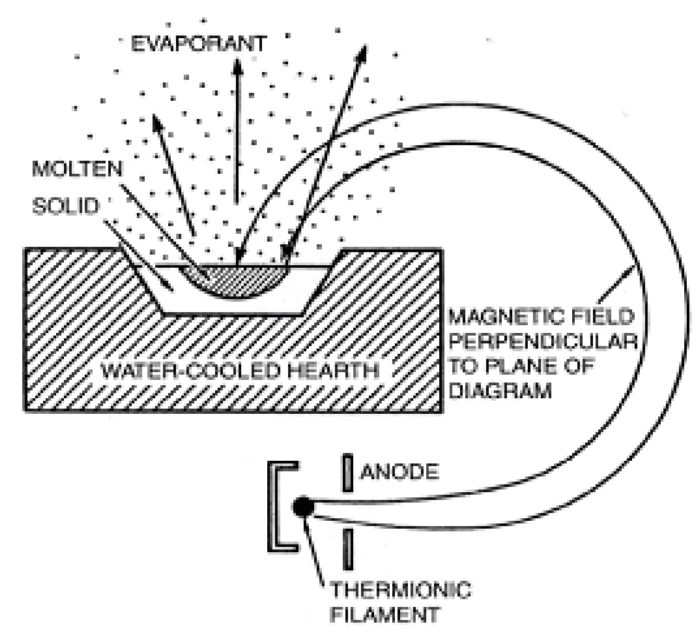

Figure 2.2: Diagram of an e-beam evaporator like the one used in this work.

\subsubsection{Magnetron Sputtering}

An important disadvantage of the deposition techniques outlined above is that they rely on evaporation. For materials with high melting or sublimation points it may be impractical or impossible to use a k-cell. By contrast, sputtering is a physical vapor deposition method that utilizes momentum transfer from energetic ions to ablate source material from a target. For this reason, sputtering finds wide industrial application for the deposition of metallic films that would be more difficult to produce by evaporation methods [43]. A diagram of a typical sputtering gun is shown in figure 2.3. In DC sputtering, the deposition chamber is filled with a plasma 
of an inert gas, typically argon or krypton. A target of the material to be deposited is mounted on a conductive backing plate to which a negative potential is maintained. The sputtering gas ions are accelerated toward the target, which acts as the cathode, and strike the surface with sufficient momentum to kinetically dislodge target atoms from the surface. Those atoms then settle on the substrate (anode) and form the desired film. The typical pressures used in sputtering range from several to hundreds of mTorr, far higher than those used in MBE. As a result, there is considerable diffusion of the target atoms as they travel to the substrate, and the deposition is consequently non-directional.

When the energized ions strike the target surface they produce emission of secondary electrons in addition to the target atoms. To take advantage of this, DC sputtering is often enhanced with the use of a magnetron, essentially a ring shaped magnet placed behind the target backing plate as illustrated in figure 2.3. The magnetic field produced by the magnetron partially confines the free electrons above the target surface [44]. The utility of this trapping is two-fold. First, the free electrons do not bombard the target as extensively as they would otherwise, resulting in less heating and damage to the target. Second, the cyclotron orbits performed by the electrons increases the distance they travel through the gas, thereby increasing the probability of their collision with and ionization of a neutral gas atom, and helping to sustain the plasma. 


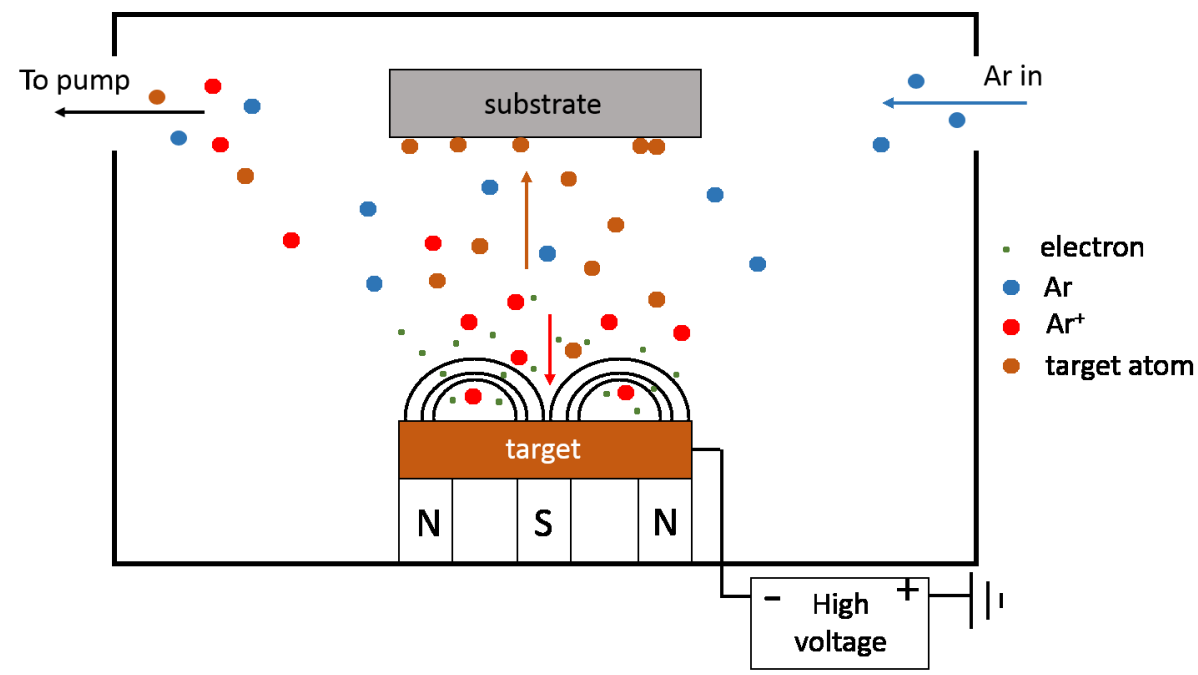

Figure 2.3: Diagramatic depiction of DC magnetron sputtering.

\subsection{Patterning and Fabrication}

\subsubsection{Photolithography}

Photolithography is a process by which a light sensitive polymer is patterned so that it can be selectively removed from desired regions. The remaining polymer can then act as a mask to protect covered areas from being altered in subsequent deposition or etching processes, and thereby facilitating the fabrication of complex devices such as integrated circuits [45]. The process begins with the application of a thin even layer of polymer, known as a photoresist or simply resist, to a sample surface. The liquid resist is applied to a sample while it is rotated normal to its surface at several thousand rotations per minute. Centrifugal forces cause the viscous resist to spread out in an even film typically on the order of a micron in thickness, with the exact thickness determined by the spinning rate, duration, and the particular resist being used. In our work, we've employed a specific form of photolithography called contact photolithography in which the pattern is transferred at a 1:1 scale 
from a physical mask as outlined in figure 2.4. The mask is made from a transparent material (quartz) with a predifined pattern layed out on it in the form of a thin film of opaque material (chrome). The mask is placed in direct contact with the surface of the resist and is then exposed to a specific dose of ultraviolet radiation. The areas of the resist exposed through the transparent regions of the mask are chemically altered in such a way that they become more susceptible (positive resist) or less susceptible (negative resist) to removal by a chemical developer. The sample is subsequently submerged in such a developer to remove the exposed/unexposed polymer, leaving behind a positive/negative transfer of the original mask pattern.

The specific parameters involved in our photolithographic processing were as follows. We used AZ 5214 photoresist (a nominally positive resist with invertibility). The resist was spun on the samples at $3000 \mathrm{rpm}$ for $60 \mathrm{~s}$ to attain a thickness of approximately $1.6 \mu \mathrm{m}[46]$. The samples were soft baked on a hot plate at $95^{\circ} \mathrm{C}$ for four minutes. The lithography was performed in a Karl Suss MA6 mask aligner with a UV wavelength of $320 \mathrm{~nm}$. The samples were exposed to a dosage of $100 \mathrm{~mJ} / \mathrm{cm}^{2}$, and then developed for $60 \mathrm{~s}$ by bathing in AZ 300 MIF developer.

\subsubsection{Etching}

In the fabrication of devices it is sometimes necessary to remove previously deposited material in a selective and controlled manner. There are two basic types of processes for doing so: wet and dry etching. In wet etching, material is removed by submerging the sample in a bath of a chemically reactive solution, usually an acid or base. This method has several potential drawbacks, namely, the same chemical may etch different materials at different rates or not at all. Additionally, a liquid etchant will act from every direction that is exposed to the etchant, for example etch- 
1.

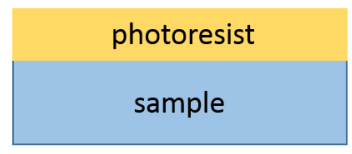

3.

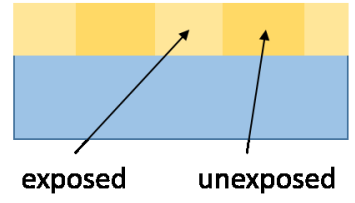

2.

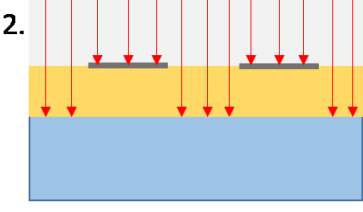

UV exposure

4.

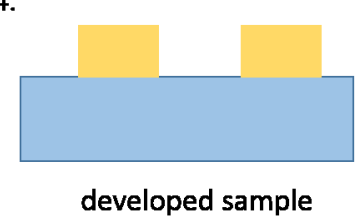

Figure 2.4: Stages of the photolithography process. Photoresist is spun into a uniform layer on the sample. Then the photomask is placed in contact with the resist and the sample is exposed to ultraviolet light. The exposed regions are chemically weakened. A chemical developer removes the exposed regions of the resist.

ing around the sides of a patterned structure. Also, the etching will continue until the reactive chemical has been removed or neutralized, making wet etches difficult to control. In dry etching, a sample is bombarded by reactive or inert ions, and material is removed by chemical and/or kinetic action of the ions with the exposed surface. This has the benefits of being easier to control, being more reliable, and usually producing a more anisotropic removal of material. In the present work, samples were patterned by means of a dry etching technique called argon ion milling, in which a beam of chemically inert Ar ions are accelerated and collimated by a grid voltage. A neutralizer filament neutralizes the ions to reducing surface charging, and the Ar atoms then bombard the sample from a uniform direction, dislodging material from the exposed surfaces via momentum transfer [45]. In the present work, films were sputtered by the Ar ion cleaning source attached to the CVC 610 DC magnetron sputtering station operated by WVU shared research facilities. Etch rates for materials used in our work are listed in table 2.1. These rates were obtained using the set 


\begin{tabular}{c|c}
\hline \hline Material & Etching Rate $(\AA / \mathrm{s})$ \\
\hline $\mathrm{Co}$ & $2.15 \pm 0.14$ \\
$\mathrm{Pd}$ & $4.92 \pm 0.03$ \\
$\mathrm{FeF}_{2}$ & $3.81 \pm 0.03$ \\
\hline \hline
\end{tabular}

Table 2.1: Etching rates obtained by Ar ion milling of pure films.

\begin{tabular}{c|c}
\hline \hline System parameter & Experimental value \\
\hline cathode current & $31.7 \mathrm{~A}$ \\
discharge voltage & $30 \mathrm{~V}$ \\
beam voltage & $400 \mathrm{~V}$ \\
accelerator voltage & $100 \mathrm{~V}$ \\
neutralizer filament current & $3 \mathrm{~A}$ \\
neutralizer emission current & $40 \mathrm{~mA}$ \\
\hline \hline
\end{tabular}

Table 2.2: Etching parameters used to obtain the etching rates in table 2.1.

of parameters shown in table 3.1 .

\subsubsection{Wire Bonding}

In virtually all modern microelectronics, such as commercially produced integrated circuits, the interconnections between the device and its packaging are made via ultrasonic wire bonding [47]. In this technique, shown schematically in figure 2.5, a thin wire of a soft metal (typically $\mathrm{Au}, \mathrm{Al}$, or $\mathrm{Cu}$ ) with a diameter of about 25 - 33 microns is fed through a capillary that forms part of a resonant structure connected to a piezoelectric driver. When the wire is brought into contact with a metallic bonding pad the capillary is vibrated at a frequency that could range from 0.1 to $300 \mathrm{kHz}$. The combination of pressure and ultrasonic energy softens the metals and pushes surface oxides and other intermetallic contaminants to the periphery, and bringing the clean metals into direct contact to form a weld within several milliseconds. Our bonding was performed using a West Bond 74776E wire bonder, using 
the following parameters. The sample was mounted on a hot plate at $90{ }^{\circ} \mathrm{C}$ to assist the bonding process. The ultrasonic power was $300 \mathrm{~mW}$, the bonding time was 25 ms, and the high(low) force of the capillary against the sample was 20(10) dynes.
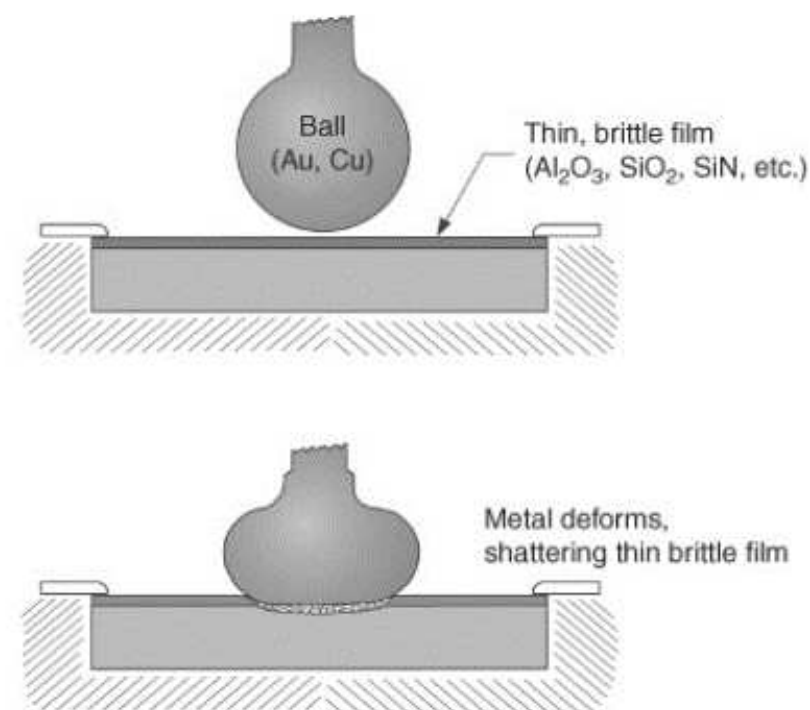

Deforming ball pushes shattered film aside into debris zones. Clean metals plus ultrasonic energy form a bond.

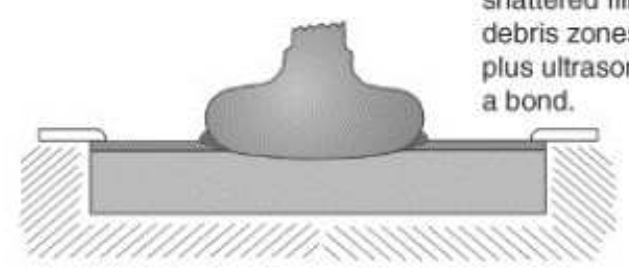

Figure 2.5: Schematic of the ultrasonic bonding process taken from reference [47].

\subsection{Characterization Techniques}

\subsubsection{Reflection High Energy Electron Diffraction}

Reflection High Energy Electron Diffraction (RHEED) is a powerful and widely utilized technique for surface analysis in UHV systems. As the name suggests, a high energy and consequently short wavelength beam of electrons is focused on a sample 
at a grazing angle of incidence, resulting in a diffraction pattern with features characteristic of the surface geometry. A typical RHEED system is shown schematically in figure 2.6. An electron gun produces a beam of electrons in the energy range of about 8 to $20 \mathrm{keV}$ which glance off of a sample at around 1 degree of incidence and are reflected onto a fluorescing screen, producing a visible diffraction pattern. Because the electron momentum is mostly in the forward direction, there is very little penetration below a few monolayers [39]. This makes RHEED a highly surface sensitive technique, and results in a diffraction pattern that is largely insensitive to out-of-plane atomic structure.

Very smooth surfaces produce RHEED patterns with vertical streaks which arises since the 2-dimensional surface lattice acts essentially like a 1-dimensional diffraction grating. The spacing between these streaks is inversely proportional to the lattice spacing in the direction perpendicular to the beam. For rough surfaces, the electron beam will pass through local areas of bulk crystal, yielding a 2-dimensional diffraction pattern visible as an array of spots. In this way, a qualitiative measurement of surface quality can be obtained. In addition to streaks and spots, RHEED patterns frequently exhibit curves known as Kikuchi lines. These are produced by Bragg diffraction of inelastically scattered electrons, and their sharpness is another qualitative indicator of sample quality. Kikuchi lines tend to rotate with the sample, Therefore orientations with highly symmetric Kikuchi patterns help to identify crystallographic symmetry axes [48]. The MBE system used to grow the materials in this work is integrated with an RH-15 electron gun and power supply from Staib Instruments operating at a power of $15.1 \mathrm{kV}$ and typical filament current of 1.4 to $1.5 \mathrm{~A}$. 


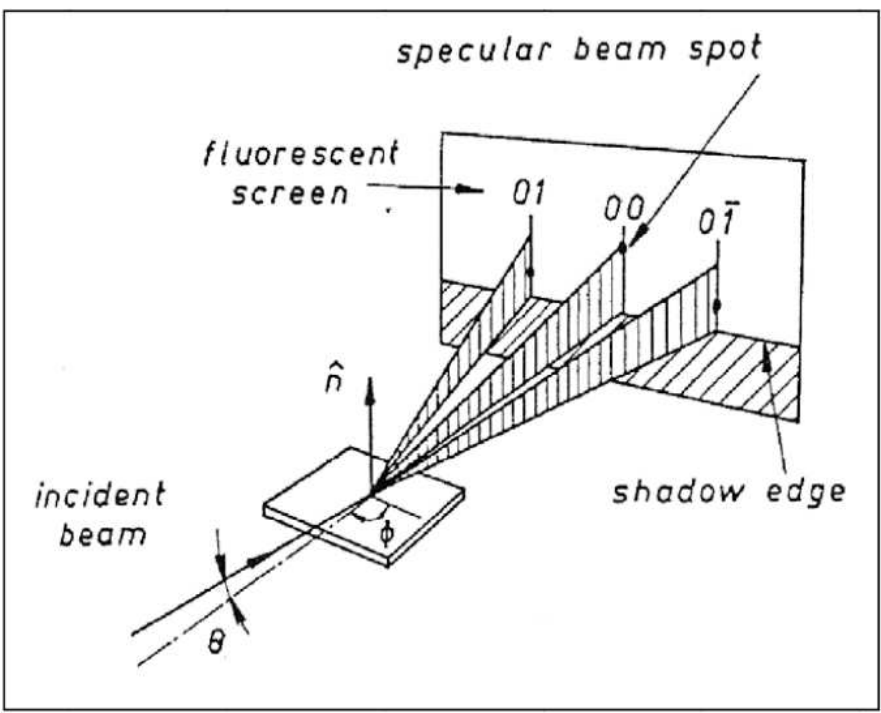

Figure 2.6: Schematic of a RHEED system [38].

\subsubsection{X-ray Diffraction}

Since it was pioneered in the early 20th century by W.H. and W.L. Bragg [49], the technique of x-ray diffraction (XRD) has become one of the most widely utilized in materials science. As x-rays have wavelengths on the same order of magnitude as the spacing between atoms, their scattering can exhibit interference patterns characteristic of the arrangement of atoms within a material. In Bragg's formulation of x-ray scattering the feature of focus is the family of parallel, nested planes formed by atomic or molecular units in a periodic lattice. For each family of planes denoted by Miller indices $\{h k l\}$ and separated by distance $d_{h k l}$, it is assumed that a beam of x-rays will undergo specular reflection from multiple planes as depicted in figure 2.7. The path difference between rays reflected from two adjacent planes is $2 d_{h k l} \sin \theta$, for an angle of reflection $\theta$. The condition for those combined waves to interfere constructively is that the path difference be equal to an integer multiple of the wavelength, leading to Bragg's law: 


$$
\lambda=2 d_{h k l} \sin \theta,
$$

where $\lambda$ is the $\mathrm{x}$-ray wavelength.

Alternatively, assuming that each atomic or molecular unit acts as an independent point scatterer of an incident beam of $\mathrm{x}$-rays with wave vector $\mathbf{k}$, it can be shown that an equivalent form of Bragg's law is:

$$
\mathbf{k}^{\prime}-\mathbf{k}=\mathbf{G}_{h k l},
$$

where $\mathbf{k}^{\prime}$ is the wave vector of the scattered beam with $|\mathbf{k}|=\left|\mathbf{k}^{\prime}\right|$, and $\mathbf{G}_{h k l}$ is a vector of the reciprocal lattice with Miller indices $\{h k l\}$ [50]. The conceptual equivalence of these two formulas can be perceived by noting that $\mathbf{G}_{h k l}$ is a vector perpendicular to the family of reflecting planes and $\left|\mathbf{G}_{h k l}\right|=2 \pi / d_{h k l}$, while the vectors $\mathbf{k}$ and $\mathbf{k}^{\prime}$ form an angle of $2 \theta$. Therefore, all of the diverse forms of x-ray crystallography have one common aim, which is map regions of the reciprocal space corresponding to that crystal by equating combinations of peak intensity with vectors of the reciprocal lattice.

There are a variety of methods for performing x-ray diffraction measurements, but the one employed in this study used a four circle diffractometer similar to that shown in figure 2.8. Scans were performed with the sample located at the center of rotation for the four angles $\omega, 2 \theta, \chi$, and $\phi$. In a conventional out-of-plane scan, $\chi$ is oriented at $90^{\circ}$ where $\chi=0^{\circ}$ is defined with the surface normal oriented vertically. A $\theta-2 \theta$ scan involves measuring the diffracted beam intensity as both $\omega$ and $2 \theta$ are varied with $\omega$ set to one half the value of $2 \theta$. This configuration is ideal for the analysis of thin films as it allows the determination of the crystal phases present in the film as well as the inter-planar spacing for those phases. The $\theta-2 \theta$ scan is conceptually diagrammed in 
figure 2.9. Here the scan amounts to sweeping through a cross section of reciprocal space in the radial direction perpendicular to the surface normal. A scan in which $2 \theta$ is constant and $\omega$ is varied is known as a rocking curve, and it sweeps out a circle in reciprocal space. Rocking curves are useful as a means of estimating crystal quality. As the reciprocal space is a Fourier transform of the real space structure, deviations from perfect periodicity in the real lattice produce broadening of the peaks in the diffracted intensity. Broadening can also be produced by angular divergence of the beam or the beam's imperfect energy distribution. Therefore, the effective width of a peak in reciprocal space, as determined from a rocking curve, is a measure of crystal coherence. For the mapping of peaks not more or less normal to the surface, in-plane scans can be performed. This entails adjusting $\chi$ away from 90 degrees or making appropriate adjustments to $\omega$ and $2 \theta$. The locations of in-plane peaks are used to determine the orientations of epitaxial films relative to the underlying substrate as well as the magnitude of the in-plane lattice parameters.

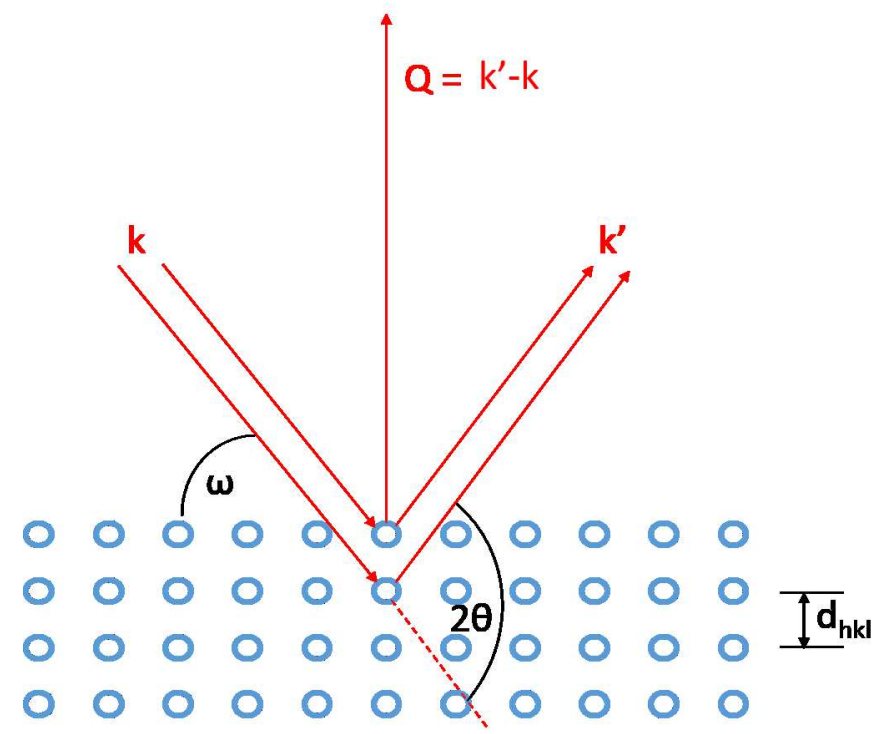

Figure 2.7: Sketch of the scattering geometry in $\mathrm{XRD}$, where $Q$ is the scattering vector and $d_{h k l}$ is the spacing between lattice planes with Miller indices $h k l$. 


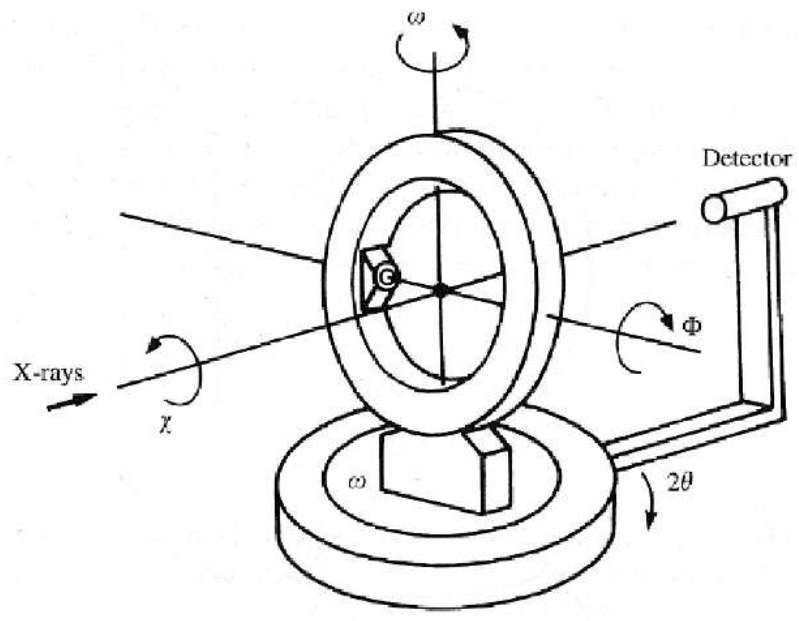

Figure 2.8: A four circle x-ray diffractometer [51].

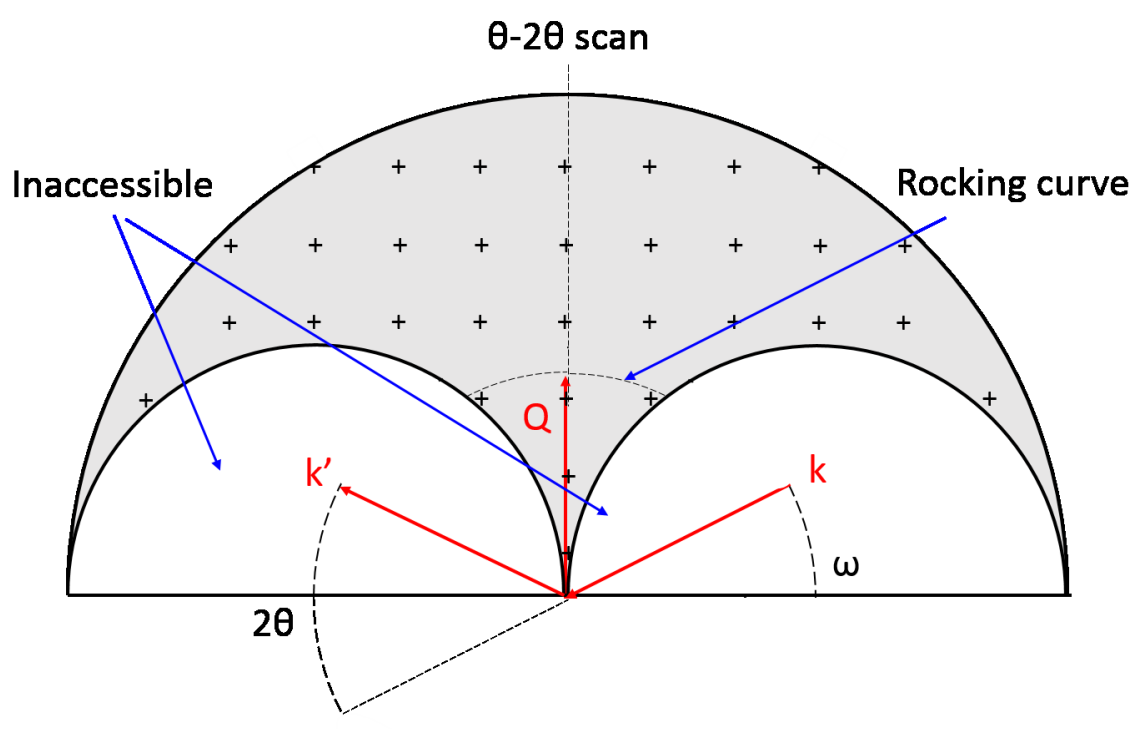

Figure 2.9: Diagram of the region of reciprocal space accessible to XRD. The $\theta-2 \theta$ scan follows the direction normal to the sample surface. Rocking curves trace an arc of constant $Q$. A tilt of $\chi$ away from $90^{\circ}$ would tilt $Q$ out of the plane of the diagram by the same angle. $\Phi$-scans sweep a circle of constant $Q$ that is centered on the out-of-plane axis, coming out of the diagram plane. Adapted from [38]. 


\subsubsection{X-ray Reflectometry}

When x-rays strike a surface at a low angle of incidence they cannot penetrate as deeply into the material, and the reflected intensity is increasingly sensitive to the optical properties of the material, independent of the crystal structure. This makes low angle x-ray reflectometry (XRR) a complementary technique to XRD for thin film structural characterization. In XRR the scattered intensity of x-rays is measured in the $\theta-2 \theta$ geometry, as in XRD, but for $\omega$ only up to several degrees. This was developed as an analytical technique by L. G. Parratt based on classical dispersion theory, and employing a recursive method for determining the intensity of specular reflection from a stack of homogeneous media with sharp interfaces [52]. Because the index of refraction for x-rays is smaller in solids than in one, there is a critical angle below which the x-ray beam exhibits total external reflection. Above the critical angle the reflected intensity drops off rapidly in a fashion heavily dependent on the roughness of the media interfaces, with rougher surfaces producing more rapid decay in intensity. For inhomogeneous materials such as thin film multilayers, the incident beam can reflect from the interfaces between layers with different optical properties. The phase differences between the various interfacial reflections produce angle dependent interference in the detected beam. This manifests itself as a series of oscillations, resulting from alternating constructive and destructive interference, known as Kiessig fringes, in the resulting $\theta-2 \theta$ scan [53].

\subsubsection{Atomic Force Microscopy}

Atomic force microscopy is a technique for mapping the surface topography of materials at the sub-micron and even down to the atomic scale. At its most basic level an AFM setup consists of a sharp probe of the order of a few nanometers in diameter 
at its tip, on the end of a cantilever held in close proximity to a sample surface by high precision piezoelectric drivers. A feedback mechanism adjusts the drivers to keep the cantilever in equilibrium as it is rastered over the surface, and the voltage applied to the piezoelectrics is translated into a mapping of the vertical displacement of the cantilever, yielding the surface topography. The cantilever deflection is typically measured by reflecting laser light off the top side of the cantilever and into a bank of photodiodes as shown in the schematic in the figure. AFM imaging is principally performed in one of two modes known as contact and tapping modes, depending upon the motion of the tip.

In contact mode, the vertical displacement of the tip relative to the sample surface is held constant by the drivers in response to deflection of the cantilever caused by the short-range interactions between the tip and the surface. This method suffers from some drawbacks, however. Collisions between the tip and surface can result in damage to the sample or tip, as well as contamination of the tip, resulting in degradation of image quality. Also, adhesive forces, particularly from adsorbed surface water under ambient conditions, can cause the tip to stick to the surface, producing inaccurate data and increasing wear on the probe tip. These issues are partially resolved by tapping mode AFM. In tapping mode the cantilever is oscillated at its natural frequency while the tip periodically comes into contact with the sample surface. Interactions between the tip and surface cause a change in the amplitude of the oscillation which is obtained from the photodiodes. The height of the cantilever is adjusted by the drivers to maintain a constant oscillatory amplitude, and hence constant average force, with the sample and the cantilever height is again registered as a map of the surface topography. Because the tip only intermittently comes into contact with the sample, this mode of operation increases the lifetime of the tip and 
reduces damage to the sample.

\subsubsection{Piezo Force Microscopy}

Piezo Force Microscopy (PFM) is a variant of AFM that allows the simultaneous imaging of ferroelectric domains as well as surface topography. It is based on the fact that all ferroelectric materials are also piezoelectrics (the converse is not generally true). Therefore, ferroelectrics exhibit the piezoelectric effect and its inverse, namely that when an electric field is applied to such a material it induces strain which deforms the material. In PFM a conductive AFM probe is used to supply an AC bias to the sample during ordinary contact AFM. If the amplitude of the applied electric field is smaller than the switching field of the ferroelectric domains, and the domains have an out of plane component, then the sample will locally expand and contract with the same frequency as the applied voltage. This produces a sinusoidal deflection of the cantilever which is superimposed on the ordinary topographical deflection. As outlined in the figure, if the applied electric field is parallel to the outof-plane polarization then the sample undergoes expansion, and if the two are antiparallel then the sample contracts. The implication of this is that if the spontaneous polarization is directed into the plane then the piezoresponse will be in phase with the applied voltage, and for out of the plane polarization it will be $180^{\circ}$ out of phase. This process is depicted in figure 2.10. The component deflections due to the piezoresponse and topography are separated by means of a lock-in amplifier with the $\mathrm{AC}$ bias used as a reference. The result is a scan showing the topographical

features and another scan of the same region with the in-phase and out-of-phase response corresponding to regions of different out-of-plane spontaneous polarization. The above procedure is essentially a read operation, but it is also possible to modify 
the setup for writing features in the sample surface by applying a sufficiently large DC field to switch the polarization. Written patterns can subsequently be read to yield confirmation of ferroelectric switching behavior.
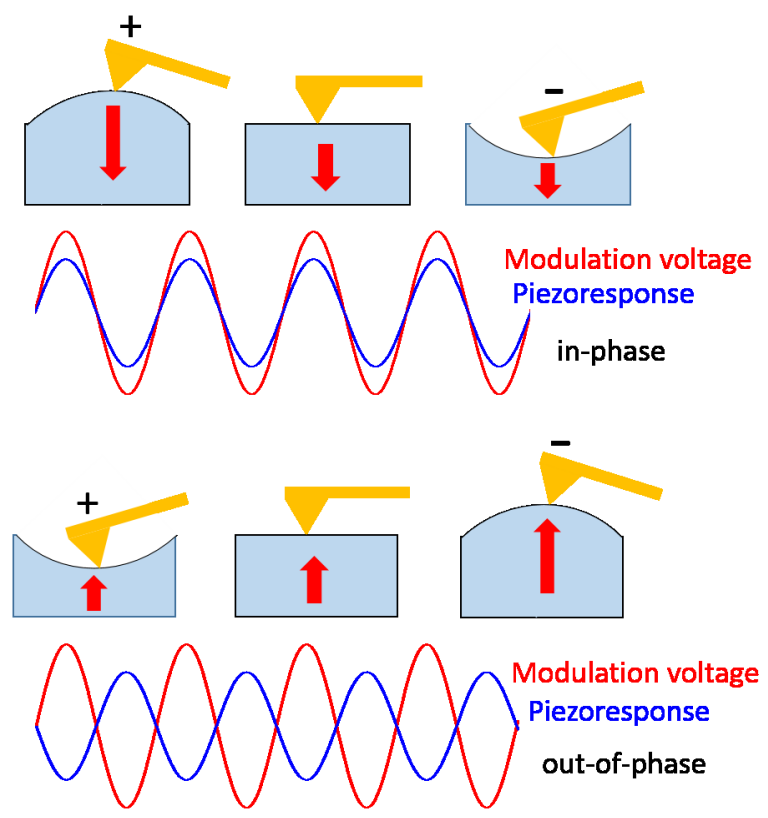

Figure 2.10: Illustration of in-phase and out-of-phase piezoresponse. The phase difference between the piezoresponse and the applied AC bias to the sample depends upon the sign of the out-of-plane component of the spontaneous polarization.

\subsubsection{Magnetometry}

Vibrating sample magnetometry (VSM) is a method of measuring the total magnetic moment of a sample based upon Faraday's law of induction. The apparatus, illustrated in figure 2.11, involves mounting the sample on the end of a rod that is suspended vertically between the coils of an electromagnet in the vicinity of a small secondary coil called a pick-up coil. According to Faraday's law, the voltage induced in the pick-up coil is proportional to the time rate of change of the magnetic flux 
through the coil. If a magnetized sample is vibrated in a magnetostatic field, there will be a contribution to the flux from the external magnet and the sample, but only the portion from the sample will have a time dependence and therefore induce a voltage in the pick-up coil.

In a typical VSM setup, the sample rod is driven into harmonic oscillation along its axis by a piezoelectric actuator operating at $10-100 \mathrm{~Hz}$ [54]. This induces a sinusoidal voltage in the pick-up coil whose magnitude is proportional to the total moment of the sample. The in-phase component of that signal is isolated by use of a lock-in amplifier with the driving voltage of the actuator as a reference. The conversion factor for voltage to magnetic moment can be obtained by measuring the induced signal from a calibration sample of known moment. By performing a series of measurements in different magnetic fields it is possible to obtain a hysteresis curve for the material under study. In the VSM system used here (PPMS with VSM option from Quantum Design) the sample rod was also enclosed in a sealed tube, allowing control over atmospheric and/or temperature conditions during measurement.

An even more sensitive form of magnetometry is based on the superconducting quantum interference device, or SQUID. There are two types of SQUID sensors, DC and RF. In this work, magnetometry was performed with the later, and so only it will be described in detail. A SQUID sensor is a superconducting loop containing either one (RF) or two (DC) Josephson junctions, i.e. thin tunnelling barriers of non-superconducting material. A dissipationless super current can flow through the junction below a critical value $I_{c}$. For currents larger than $I_{c}$ the junction becomes resistive and a potential difference develops across the barrier. For a superconducting loop, the periodic boundary condition on the coherent state wave function implies the quantization of the magnetic flux trapped in the loop. It can be shown that when 


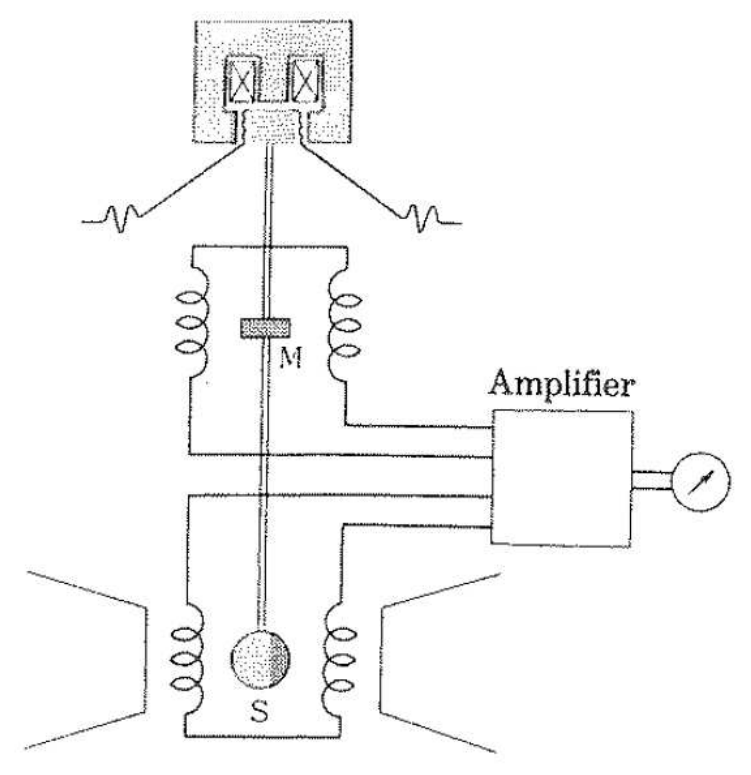

Figure 2.11: Diagram of a vibrating sample magnetometer. The sample $S$ is vibrated in a magnetic field. The voltage induced in the pick-up coil (feeding into the amplifier) is compared with one induced in a standard sample $M$ to determine the magnetic moment of $S$. Taken from reference [54].

a Josephson junction is introduced into such a loop, the super current is periodic in the total flux according to

$$
I=-I_{c} \sin \left(2 \pi \Phi_{T} / \Phi_{0}\right),
$$

where $\Phi_{0}$ is the flux quantum and $\Phi_{T}$ the total flux [55]. The total flux, being a combination of the externally applied flux $\Phi_{E}$ and flux produced by the loop itself, can be expressed by the nonlinear equation

$$
\Phi_{T}=\Phi_{E}-L I_{c} \sin \left(2 \pi \Phi_{T} / \Phi_{0}\right),
$$

where $L$ is the self inductance of the loop.

In the RF SQUID, such a loop is inductively coupled to an LC circuit driven at 
resonance in the RF regime. If the current in the resonant circuit is high enough, it will induce a sufficiently high current in the SQUID to reach the critical value and dissipate energy on each cycle. That energy is ultimately supplied by the resonant circuit. If additional flux is supplied to the SQUID from an external source the RF drive will not have to supply as much current in order to induce a critical current. If the RF current is kept constant this manifests itself as a modulation in the RF voltage which is periodic in the externally applied flux with period $\Phi_{0}$ [56]. In this manner the SQUID effectively acts as a sensitive flux to voltage converter.

In this work, the SQUID magnetometry measurements were performed with a Magnetic Properties Measurement System (MPMS) from Quantum Design. The physical arrangement of the sample is very similar in this instrument to that in the VSM. A sample is mounted on the end of a rod that is positioned vertically between the coils of an electromagnet. The magnetic moment of the sample is inductively coupled to a superconducting pick-up coil in a second derivative gradiometer configuration that is designed to eliminate signals from the superconducting magnet or stray magnetic fields. The pick-up coil is in turn inductively coupled to the SQUID sensor. The sample is then vertically translated through the pick-up coil, ultimately inducing a voltage response that has been factory calibrated to the magnetic moment of the sample.

\subsubsection{Magnetoresistance}

Measurement of the magnetoresistance was made using a six contact Hall bar geometry as shown in figure 2.12. The Hall bar was patterned by photolithography and Ar ion milling as described above. A constant current was fed through the main channel from the contacts on the ends of the bar. Then the potential difference be- 
tween adjacent longitudinal contacts was measured from a high precision voltmeter. The samples were mounted in a cryostat placed between the coils of an electromagnet such that the applied magnetic field was oriented in the plane of the sample and parallel to the current flow. Such measurements were taken with the external magnetic field cycled through a hysteresis loop in order to determine the effective coercive field of the ferromagnetic films by the associated drop in resistivity that occurs during magnetization reversal.

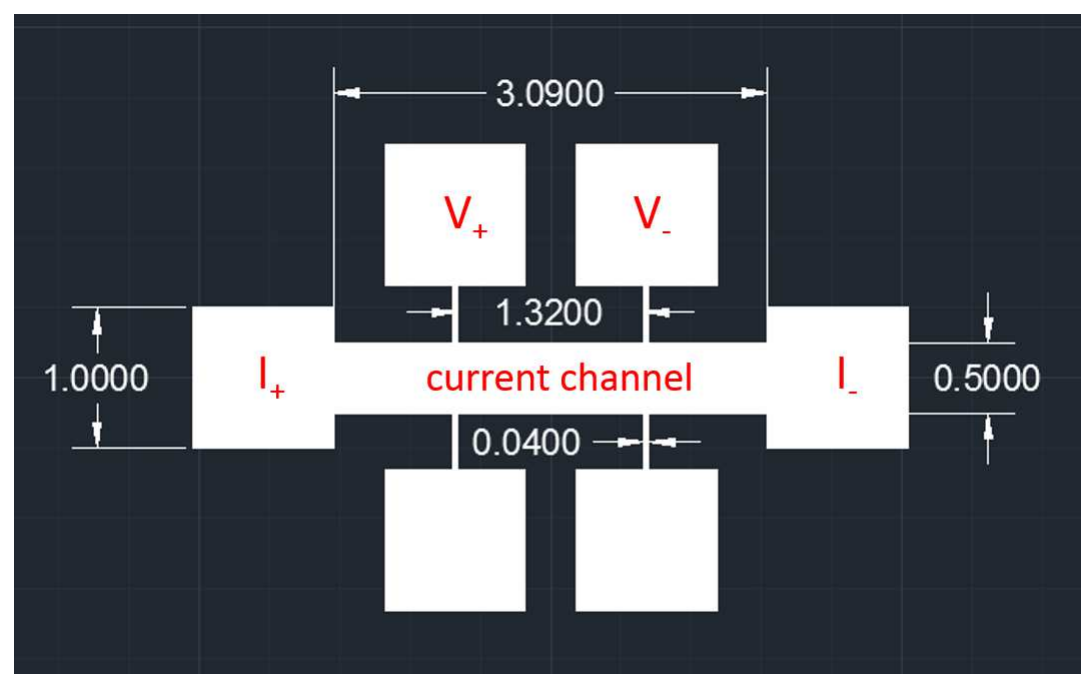

Figure 2.12: Photolithography mask design used for patterning of six contact Hall bar. All units in $\mathrm{mm}$.

\subsubsection{Ferroelectric Properties Testing}

The essential characteristics of interest for ferroelectric materials are contained in their ferroelectric hysteresis loops, namely the spontaneous polarization, saturation polarization, and coercive field. To measure these quantities a variety of methods have been devised, one of the earliest of which was developed by Sawyer and Tower in 1930 [57]. A diagram of the Sawyer-Tower circuit is shown in figure 2.13(a). In their method, the ferroelectric material being tested is used as the dielectric in a 
capacitor, which is placed in series with a reference capacitor $C$. The polarization of the ferroelectric is the charge density on the corresponding capacitor. Since the two capacitors are in series, the charge on each of them must be the same. By measuring the potential drop $V$ across the the reference capacitor, the charge, $Q=C V$ can be determined, and hence the polarization of the sample. A significant drawback of this method is due to the so called "back voltage" effect, where charge accumulation in the reference capacitor generates a voltage that counteracts the drive voltage applied to the test capacitor. To reduce this effect, a reference capacitor must be used which is much larger than that of the test device. Additionally, cables and probes connecting to the test device can contribute a parasitic capacitance that adds in parallel to the reference capacitor, effectively changing its capacitance to an unknown value.

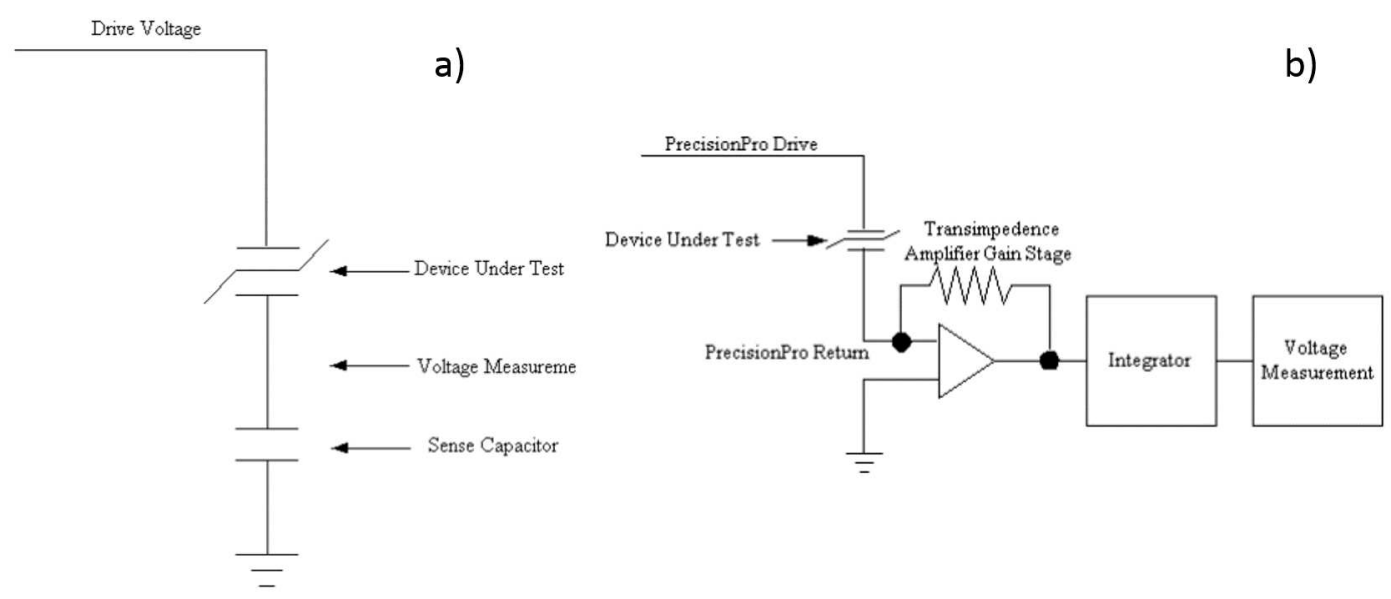

Figure 2.13: a) Schematic of a basic Sawyer-Tower circuit, and b) the virtual ground circuit used for ferroelectric testing in the present work [58].

Ferroelectric measurements in this work were performed using a Radiant Technologies Precision LC tester, which operates with a modified Sawyer-Tower circuit in which the reference capacitor is replaced by the circuit shown in figure 2.13(b). 
In this circuit, the output from the test capacitor is fed into the inverting input of an operational amplifier which forms a "virtual ground," preventing back voltages and reducing the influence of parasitics. The amplifier output, which is proportional to the current in the sample, is then integrated to yield the charge that that has flowed to the test capacitor. Since the charge is obtained through integration of the current, there are various contributions to the resulting hysteresis loop that are not related to the ferroelectric polarization. One is linear charging of the capacitor with applied voltage that can occur either due to the linear dielectric properties of the test material or due to parasitic capacitance of the cables connecting the test device. Additionally, dielectric loss, which is quadratic in the applied voltage, can produce an opening in the resulting hysteresis loop that can be easily mistaken for ferroelectric switching $[24,59]$. To eliminate these contributions our measurements were taken in remanent mode.

A conventional hysteresis measurement (shown in figure 2.14) is preceded by a pre-set voltage pulse whose purpose is to set the polarization of the sample in the direction opposite to the electric field that will be applied in the first leg of the measured loop. This is done to ensure that the intrinsic polarization is in a position to be switched during the measurement. The switching manifests itself as pulse of current to the test capacitor. If the pre-set pulse is applied in the same direction as the first leg of the measurement then no such switching behavior will occur. Clearly, the resistive and ordinary capacitative behavior of the test device should be independent of the sign of the pre-set pulse. The remanent hysteresis loop is constructed by taking the difference between switching and non-switching loops to extract the contribution to the overall signal that is due to ferroelectric behavior. Because the second half of a full hysteresis loop will always be switched, the remanent 

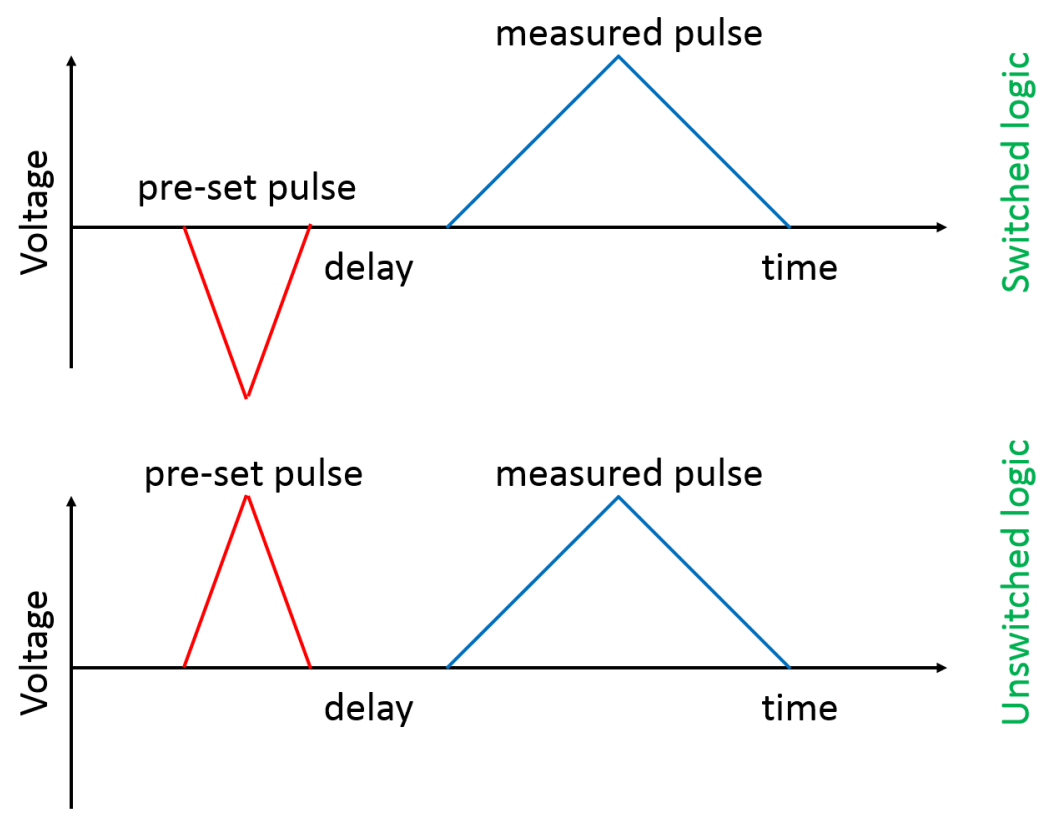

Figure 2.14: Drive voltage vs. time characteristic for switched (top) and unswitched (bottom) hysteresis measurements. Similar measurements are taken with the sign of the measured pulse reversed to construct the full hystersis loop. Adapted from reference [58].

loop is constructed by taking half loops with positive and negative bias as separate measurements. The tester software then combines them into a single graph. 


\section{Chapter 3}

\section{Exchange Bias in $\mathrm{Co} / \mathrm{FeF}_{2} / \mathrm{Co}$}

\section{Trilayers}

\subsection{Introduction}

In this study, we research the magnetic switching properties of doubly exchange biased tilayers consisting of the FM's separated by an AF spacer. The original motivation for this study was the fabrication of the novel MTJ architecture with an insulating AF as the tunneling barrier. While AF's are commonly used in MTJ's to pin one of the FM electrodes, the goal behind the doubly biased structure was to enhance the functionality of the device by making a structure whose tunneling characteristics could be tuned via transition across the Néel temperature. Additionally, such a device would be an ideal means by which to probe the excitation spectrum of inelastic spin waves [60] in the AF. Ultimately, a functional device was never completed, but the prerequisite fabrication of a trilayer film structure carries with it its own set of interesting questions related to $\mathrm{EB}$, such as: what is the relative contribution between the bulk AF and the interface? 
Most studies of EB in thin film systems focus on bilayer structures. A limitation of this approach is that, although it is the uncompensated moments at the interface that produce exchange anisotropy, bilayers provide limited insight into the bulk AF structure that is responsible for pinning those moments. An advantage to coupling both AF surfaces in a trilayer structure is that it can provide clues to that bulk structure, and how the interfacial coupling is propagated through the AF film. There have been a number of studies of EB in trilayers, but due to the peculiarities of each study it is difficult to draw general conclusions. For example, Yang et al. [61] reported the formation of a spiraling AF structure in the FeNi/FeMn/Co system [ref], however it was later determined by Leung et al. that this structure resulted from unique cooling procedures [62] rather than being a general feature of trilayers. Sankaranarayanan et al. performed a detailed study of the thickness dependence of all three layers in $\mathrm{FeNi} / \mathrm{FeMn} / \mathrm{FeNi}$ trilayers, and found that EB was significantly larger at the bottom interface [63]. However their result is complicated by the tendency of FeMn to switch from the AF $\gamma$-fcc phase to non-AF $\alpha$-bcc. These examples are illustrative that each system has many unique variables that may play a role in the determination of EB. Therefore, we have studied the relatively simple system with the same material chosen for the top and bottom FM layers, and the Ising-like $\mathrm{AF}, \mathrm{FeF}_{2}$ as the spacer.

\subsection{Experimental Procedures}

\subsubsection{Growth}

Pre-diced, single side polished $5 \times 10 \times 0.5 \mathrm{~mm} \mathrm{Al}_{2} \mathrm{O}_{3}(11 \overline{2} 0)$ single crystal substrates were degreased for 10 minutes in separate baths of acetone and methanol, 
and subsequently annealed in air at $1400{ }^{\circ} \mathrm{C}$ for 3 hours to remove organic surface contaminants and reconstruct the surface. The substrates were then mounted with the polished side facing up on a 1/16 inch thick molybdenum block and secured in position by molybdenum clips. The sample mount was then transferred to a vacuum chamber with a base pressure of $10^{-9}$ Torr, where the back side of the block was heated to $750{ }^{\circ} \mathrm{C}$ for 40 minutes before being cooled back to room temperature.

Trilayer films consisting of first FM $\mathrm{Co}(20 \mathrm{~nm})$, then $\mathrm{AF} \mathrm{FeF}_{2}\left(t_{A F}\right)$ and $\mathrm{Co}(5 \mathrm{~nm})$ were sequentially deposited via electron beam evaporation at room temperature. Finally a $5 \mathrm{~nm}$ Pd layer was deposited to prevent oxide formation in the top Co layer. Prior to each deposition, the deposition rate at the sample location was determined by a QCM which could then be withdrawn before moving the sample to approximately the same position. During the deposition of each layer the sample mount was rotated at 50-60 rpm around the surface normal to promote uniformity in layer thickness. The resulting films were all polycrystalline, as confirmed by in-situ RHEED images captured after each layer deposition, showing a characteristic ring pattern, as seen in figure 3.1. A series of such samples was grown in which the nominal AF thickness $t_{A F}$ was varied $\left(t_{A F}=1,3,5,7\right.$, and $\left.10 \mathrm{~nm}\right)$ while maintaining the same approximate thicknesses for the other layers. Bilayer samples of $\mathrm{Co}(20 \mathrm{~nm}) / \mathrm{FeF}_{2}\left(t_{A F}\right)$ were also grown with $t_{A F}=3,5$, and $7 \mathrm{~nm}$, under the same conditions as above excepting that the bilayers were capped with $10 \mathrm{~nm}$ of $\mathrm{MgF}_{2}$ in lieu of $\mathrm{Pd}$.

\subsubsection{Structural Characterization}

The film surface quality was analyzed by AFM, which showed that the films grew very smoothly with root mean squared roughness less than $1 \mathrm{~nm}$. Figure 3.2 shows an AFM image for a representative sample with clearly visible terraced structures in the 


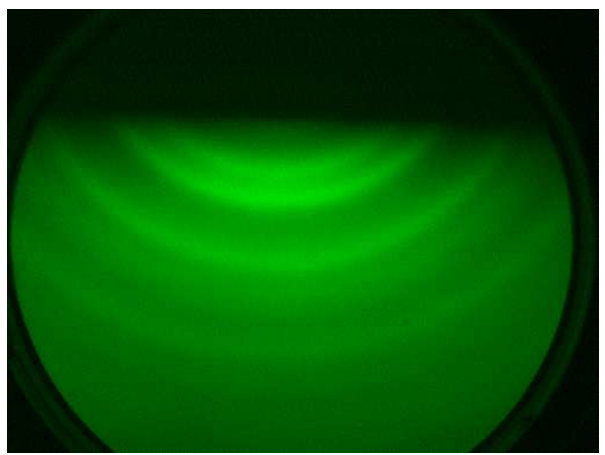

Figure 3.1: RHEED image of trilayer sample showing rings associated with the polycrystalline structure of the surface.

surface topography. The terraces are atomic steps associated with the reconstructed surface of the annealed sapphire, which are still visible despite having, in this case, over $35 \mathrm{~nm}$ of material deposited on top. This could only be possible if the growth of each layer produced a smooth surface on which to grow the next layer.

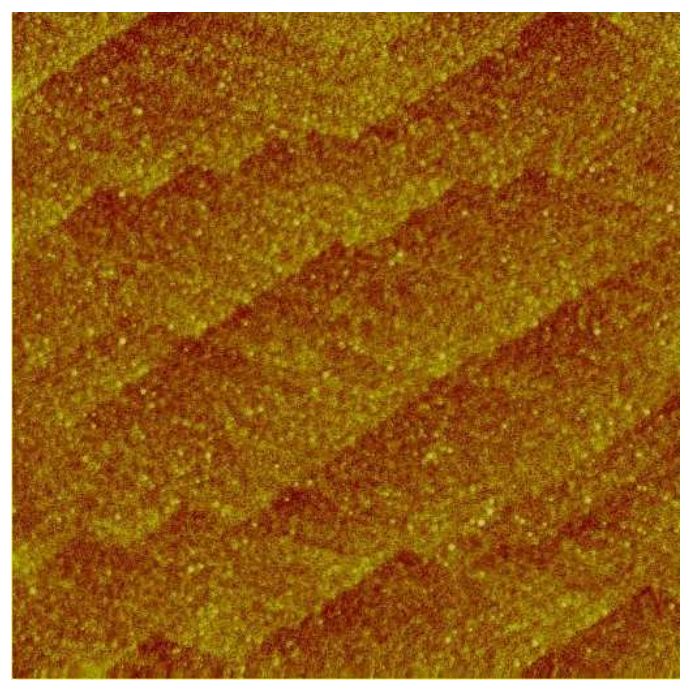

Figure 3.2: AFM image of $t_{A F}=3 \mathrm{~nm}$ sample with the steps in the underlying substrate topography visible.

Quantitative determination of the thickness and interfacial roughness for each layer was performed by analyzing x-ray reflectivity (XRR) data. XRR data taken from a $\mathrm{Cu} K_{\alpha}$ source was fitted using the open-source program GenX [64], to obtain 


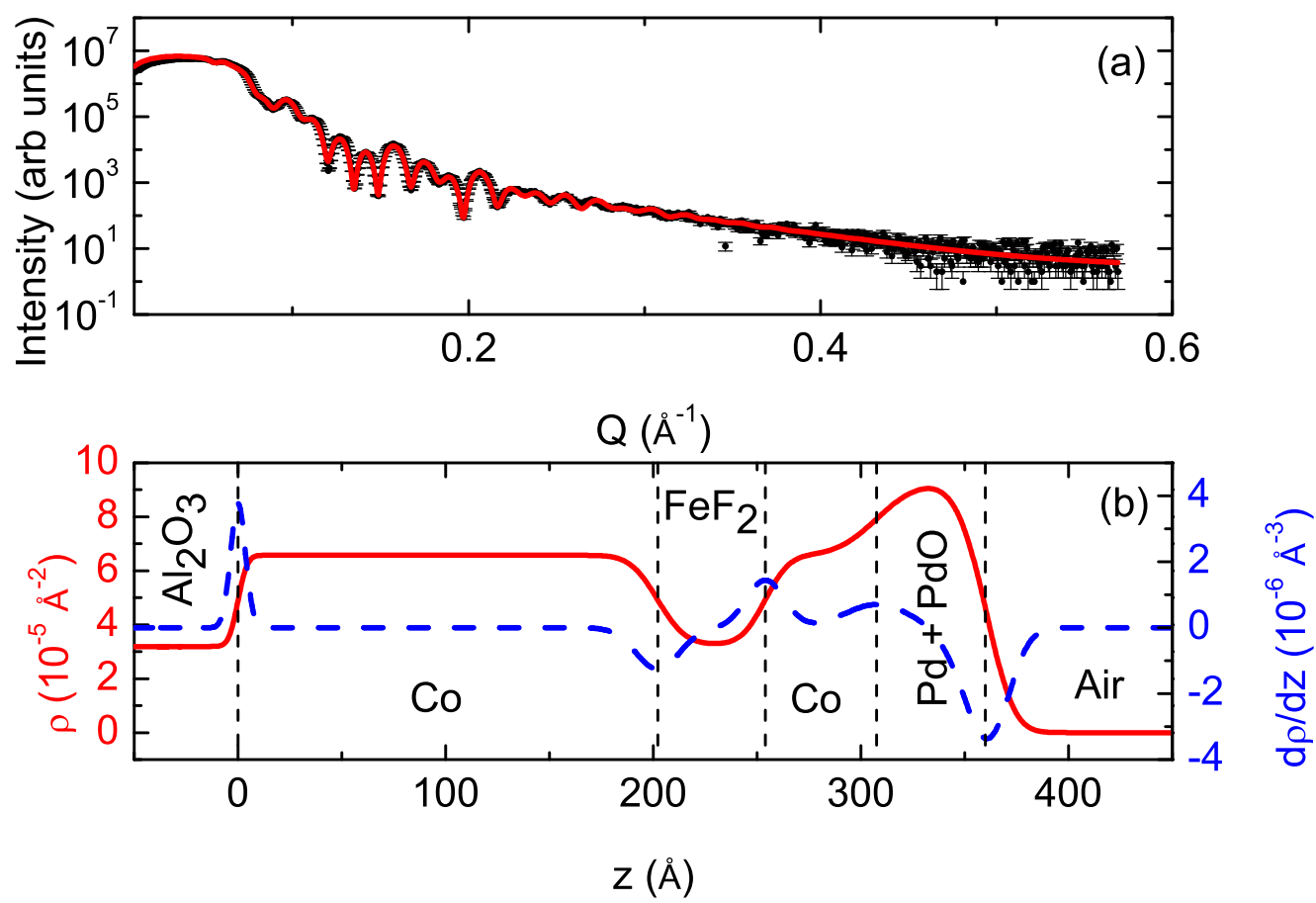

Figure 3.3: SLD profile and first derivative for a repesentative sample.

a scattering length density (SLD) depth profile for each sample. GenX uses Parratt's recursive method for determining the reflected specular intensity curve for a given structure in which parameters such as layer thickness, roughness, etc. are treated as fitting parameters. It then utilizes a genetic algorithm (a type of computational heuristic modeled on the operation of natural selection in biological evolution) to optimize the fit of the the calculated intensity to the experimental one. The fitting model employed included a surface layer of $\mathrm{PdO}$ in the trilayer samples, which the fits show formed to a depth of about $1 \mathrm{~nm}$. Figure 3.3 shows a representative example of an XRR fit and its associated SLD profile $\rho(z)$ where the depth $z$ is taken to be zero at the substrate/film interface. The thickness of each layer was determined by taking the interfaces to be defined by the peaks in the $d \rho / d z$ curve, and the interfacial 


\begin{tabular}{c|cccccccc}
\hline \hline$t_{A F}(\mathrm{~nm})$ & $t_{C o 1}(\AA)$ & $\sigma_{C o 1}(\AA)$ & $t_{F e F 2}(\AA)$ & $\sigma_{F e F 2}(\AA)$ & $t_{C o 2}(\AA)$ & $\sigma 4_{C o 2}(\AA)$ & $t_{P d}(\AA)$ & $\sigma_{P d}(\AA)$ \\
\hline 1 & 216.2 & 13.8 & 11.5 & 11.5 & 45.1 & 12.1 & 46.6 & 11.4 \\
3 & 207.6 & 11.8 & 29.2 & 9.9 & 56.1 & 11.6 & 47.7 & 9.3 \\
5 & 202.1 & 10.5 & 51.8 & 9.2 & 53.0 & 14.4 & 53.1 & 10.9 \\
7 & 214.9 & 10.0 & 69.1 & 10.4 & 60.5 & 11.9 & 47.7 & 10.6 \\
10 & 209.8 & 12.0 & 101.4 & 12.4 & 56.5 & 18.1 & 56.5 & 13.6 \\
\hline \hline
\end{tabular}

Table 3.1: Thickness and roughness parameters for trilayer samples obtained from SLD profile. Co1 is the bottom FM layer and Co2 is the top one. $\sigma$ is the roughness at the top interface of the corresponding layer defined as the standard deviation of the peak in $d \rho / d z$ representing the given interface. See figure 3.3.

roughnesses by the standard deviations of the associated peaks. The results of this structural analysis, summarized in the table 3.1 , indicate that the layers are generally well defined, and their thicknesses are in good agreement (generally within 10\%) of their nominal values. Also, the roughness observed via XRR is probably mostly limited to interdiffusion while the step disorder between adjacent layers is likely smaller per the AFM surface roughness shown in figure ??

\subsubsection{Magnetic Characterization}

The magnetic moment of the trilayers was measured as a function of temperature and applied magnetic field via vibrating sample magnetometer operating at $40 \mathrm{~Hz}$. Magnetic hysteresis loops were measured after cooling in a saturating magnetic field of $5 \mathrm{kOe}$ to well below the Néel temperature of $\mathrm{FeF}_{2}$ at $T_{N}=78 \mathrm{~K}$. The measurements were obtained by taking the average moment while continuously sweeping the field. This procedure produced near identical results to the more time consuming one of stabilizing the field for each measurement. Loops were taken between $\pm 5 \mathrm{kOe}$ from 5 $\mathrm{K}$ up to above the blocking temperature $T_{B}$ in $5 \mathrm{~K}$ increments. While training effects were not systematically studied, it was noted that during testing of the continuous sweep vs stable field measurements that two consecutively performed hysteresis loops 
taken at $5 \mathrm{~K}$ on the $3 \mathrm{~nm}$ trilayer sample showed no apparent training. The zero-field thermal remnant magnetization (TRM) was also measured vs temperature from $5 \mathrm{~K}$ to $300 \mathrm{~K}$ after cooling in a field of $5 \mathrm{kOe}$. Magnetic hysteresis loops and TRM were also measured for the bilayers under the same temperature and field conditions as the trilayers, however the measurements were made via SQUID magnetometry.

\subsubsection{Electrical Characterization}

As a preliminary procedure to performing electrical measurements on the trilayer samples, the length of the substrates had to be reduced to fit in a sample mounting setup. The film surface was spun with photoresist for protection and cut with a Disco DAD3240 wafer dicer, reducing the length of each sample from $10 \mathrm{~mm}$ to 7.5 $\mathrm{mm}$. They were subsequently patterned by photolithography and the films etched by Ar ion-milling down to the substrate, into the conventional six contact Hall bar shown in chapter 2. Using another photolithographic step, the sample was masked with photoresist everywhere except on the contact pads. $\operatorname{Ti}(10 \mathrm{~nm}) / \mathrm{Au}(200 \mathrm{~nm})$ was sputtered over the surface, and liftoff performed by immersion in acetone. The patterned samples were then mounted in the socket of an integrated circuit package with GE varnish. Ball and crescent wire bonding was used to make electrical contacts between the $\mathrm{Ti} / \mathrm{Au}$ contact pads on the film and appropriate pins on the package. The package was mounted in a corresponding socket wired to the electrical instrumentation. The underside of the package was thermally anchored to a copper plate with silver paint, and the whole assembly placed in thermal contact with the cold finger of cryostat. The samples were mounted in the cryostat so that a magnetic field could be applied in the plane of the sample and longitudinally with respect to the Hall bar, i.e. parallel to the applied current. After field cooling in $5 \mathrm{kOe}$ to $14 \mathrm{~K}$ 
the longitudinal voltage was measured as a function of applied field in a hysteresis loop from positive to negative $5 \mathrm{kOe}$, while applying a constant current of $200 \mathrm{~mA}$. The voltage could be measured across two sets of contacts as a redundancy in case of a bond failure.

\subsection{Results and Discussion}

Low temperature magnetic hysteresis loops taken on the trilayer samples show two distinct, negatively exchange biased loops, one with a small coercivity and the other with a large one, which can be clearly associated with the thick and thin Co layers respectively, due to the roughly 4 to 1 ratio in the measured moments. The exception to this trend is the $t_{A F}=1 \mathrm{~nm}$ sample, for which the two layers cannot be resolved at any temperature. It is worth noting that in both the $t_{A F}=1$ and 3 nm samples the SLD in the $\mathrm{FeF}_{2}$ layer never reaches its bulk value, suggesting that the region of interdiffusion with Co overlaps with the overall AF layer thickness (see Figure 3.4). Both the exchange field $\left(H_{E B}\right)$ and coercive field $\left(H_{C}\right)$ for the two layers are markedly different and highly temperature sensitive, with the thinner $5 \mathrm{~nm}$ layer displaying larger $H_{E B}$ and $H_{C}$. As temperature is increased $H_{E B}$ decreases until vanishing at $T_{B}$, and $H_{C}$ decreases until the two loops effectively merge. Well above $T_{B}$, the coercive fields of both layers were almost identical for all samples and could not be resolved at room temperature. The thicker layer also displays some coercivity enhancement near $T_{B}$ in each case. The results of these measurements for each of the samples are summarized in the Figure 3.5. We see that $T_{B}$ increases with $t_{A F}$, with the largest sensitivity to temperature being apparent for smaller AF thickness. The larger the value of $t_{A F}$ the closer $T_{B}$ approaches the bulk Néel temperature of $78 \mathrm{~K}$. 


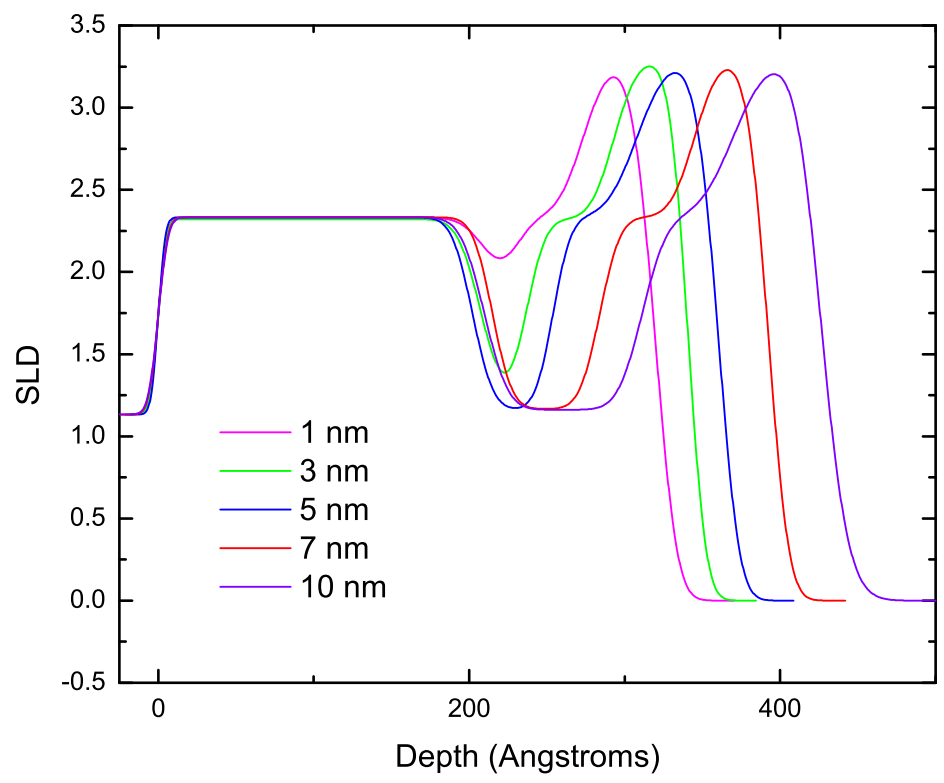

Figure 3.4: SLD profile of each of the trilayer samples. Note for 1 and $3 \mathrm{~nm}$ the SLD never reaches its bulk value.

While it is difficult to see totally consistent relationships between all the samples and their corresponding $H_{E B(T)}$ and $H_{C}(T)$ curves, there are some general observations that can be made. Most strikingly, the coercivity (see Figure 3.6) below $T_{B}$ of the thin Co layers is significantly larger than that of the thick ones. Within each set we see a similar trend with coercivity undergoing a dip in its dependence on $t_{A F}$. In the set of thin layers the low point occurs at $t_{A F}=5 \mathrm{~nm}$ while for the thick layers it is at $7 \mathrm{~nm}$. Also the absolute sensitivity of $H_{C}$ to AF thickness is more pronounced for the thin Co films.

Examination of the exchange bias vs. temperature (Figure 3.7) shows results which are qualitatively similar to those for the coercivity. The thinner layers display markedly larger exchange bias than the thick ones for all values of $t_{A F}$. The bias for the two layers drop off with increasing temperature until going to zero at the blocking 


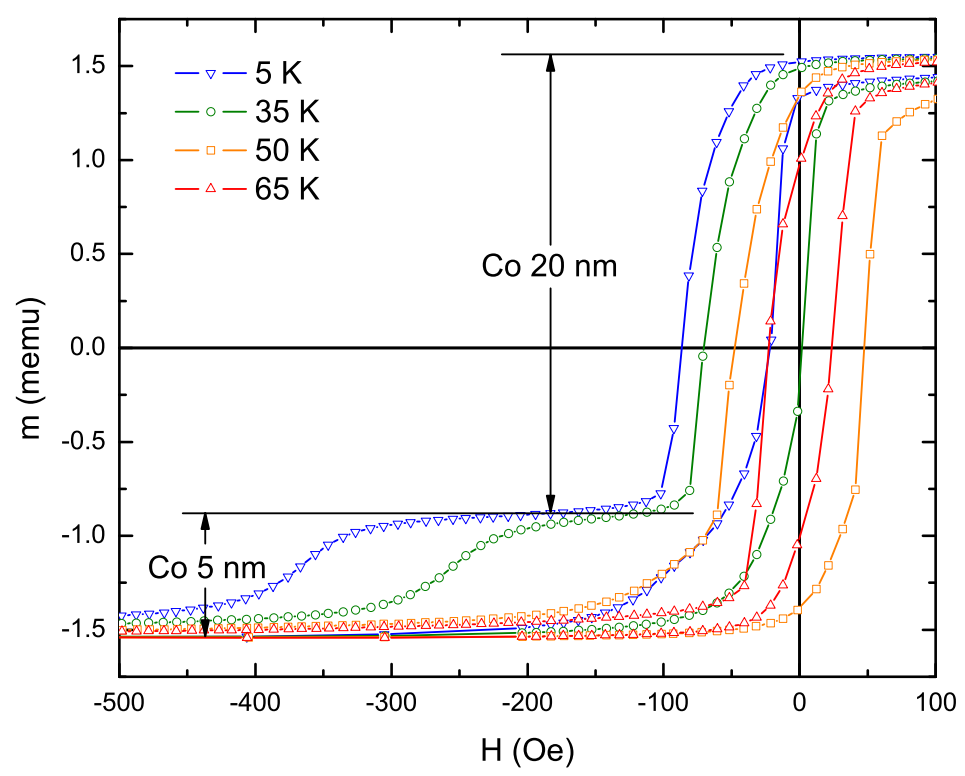

Figure 3.5: Magnetic hysteresis loops for $t_{A F}=3 \mathrm{~nm}$ trilayer sample taken for several temperatures below and above $T_{B}$.

temperature. Given the sizable gap in mean exchange field $H_{E B}$ between the thick and thin Co layers, it is clear that the dominant contribution to the magnitude of the bias is the thickness of the FM. This is not unexpected, since the exchange anisotropy is an interfacial effect whereas the Zeeman and magnetocrystalline anisotropy energies scale with the volume. It is the assumption that the interface is independent of the bulk AF structure that led Meiklejohn and Bean to predict the standard inverse relationship between the exchange field and FM thickness. That is, if the exchange energy per unit area of interface

$$
\mathscr{E}_{e x}=H_{E B} M_{F M} t_{F M}
$$

is constant then $H_{E B} \propto 1 / t_{F M}$. More at the heart of the matter is the energy 


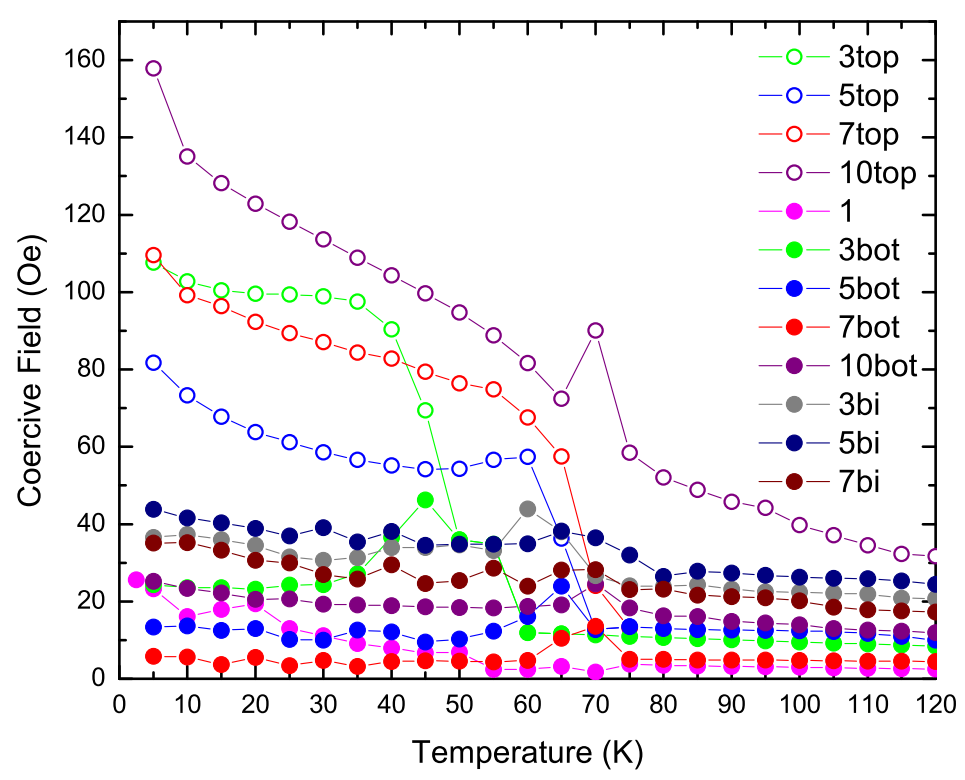

Figure 3.6: Coercive field vs. temperature. Samples are labeled with nominal AF thickness followed by top for the thin top FM, bot for the bottom FM layer, and bi for the corresponding bilayer samples.

associated with the exchange anisotropy and its variation with AF layer thickness.

Our data does not yield a direct measurement of the magnetization, but rather the total moment of the sample. Therefore we replace the term for interface magnetization $M_{F M} t_{F M}$ from Equation 3.1 with $m_{\text {layer }} / A$, where $m_{\text {layer }}$ is the total moment of each FM layer and $A$ is the area of the film. The variations in layer thickness should be implicitly contained in the total moment of each layer, i.e. moment should be proportional to thickness. In this regard, the choice of $\mathrm{Pd}$ as a capping layer was not optimal. Pd/Co interfaces are well known to favor the formation of an interfacial magnetic layer in the $\mathrm{Pd}[65]$ that could alter total magnetic moment of the thin layer. To determine if the magnetization of the top Co layer was affected by the presence of $\mathrm{Pd}$, the net moments of each layer were determined from $5 \mathrm{~K}$ hysteresis curves. The 


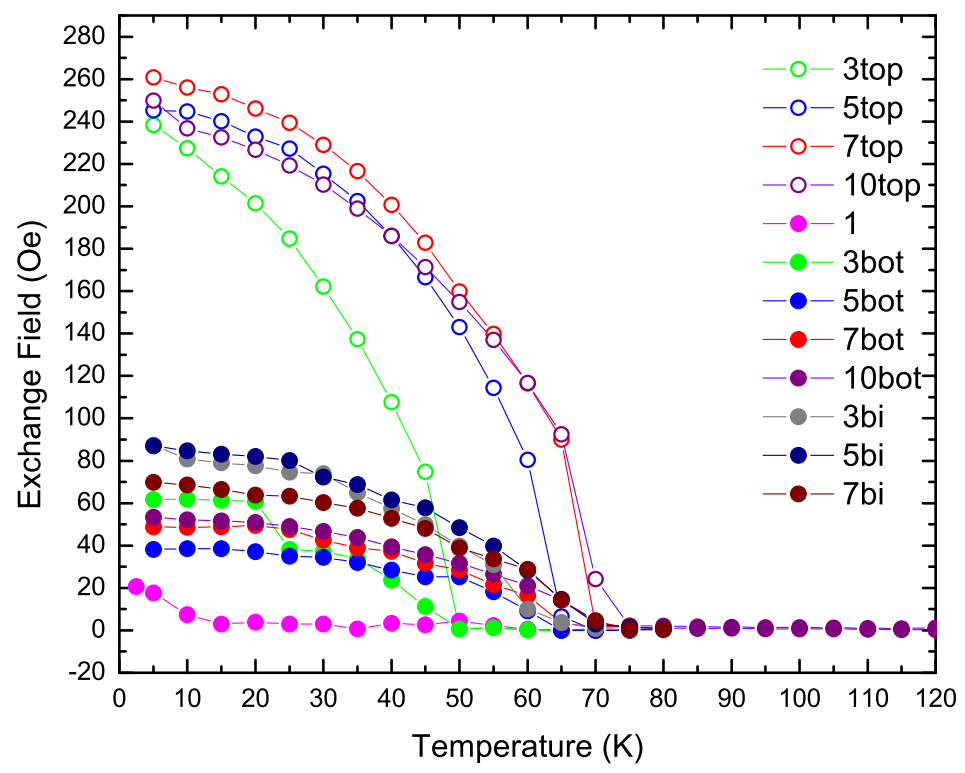

Figure 3.7: Exchange field vs. temperature.

net moment of the sample at saturation is easily determined. The boundary between the two layers was taken to be twice the moment of the hard layer at its exchange field. Table 3.2 shows the ratios of the thick(bottom) to thin(top) layer thicknesses, as determined from x-ray fits, and moments measured from hysteresis curves. We see that these ratios match reasonably well, but with the exception of the $t_{A F}=7$ nm sample the magnetic moment ratios are a little smaller than the thickness ratios. Therefore, it would appear that the Pd was probably slightly magnetized, but we conclude that the $\mathrm{Pd} / \mathrm{Co}$ interface does not significantly alter the magnetization in the accompanying Co layer, and our procedure for calculating $\mathscr{E}_{e x}$ is still a valid one.

The exchange energies per unit area calculated using $\mathscr{E}_{e x}=H_{E B} m / A$ are shown in figure 3.8. If it were in fact the case that the coupling energy at a given AF/FM interface was independent of the thickness of either film, then it would follow that 


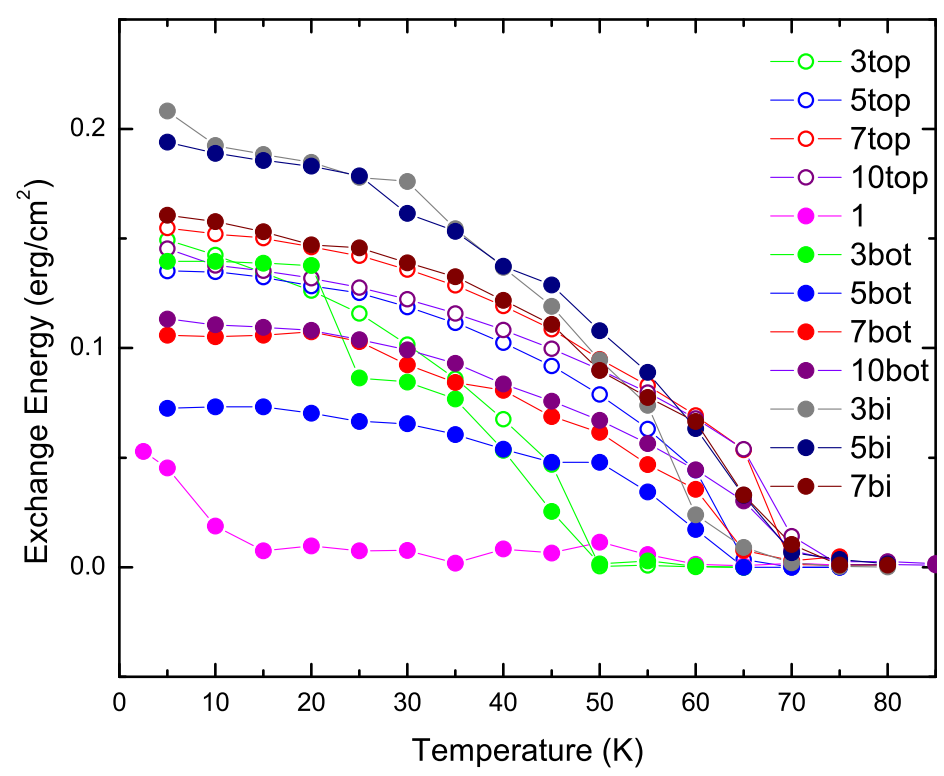

Figure 3.8: Interfacial exchange energy vs. temperature.

the energy vs. temperature curves should collapse on top of each other. However, as we see in the figure, this is not the case for most samples. In the $t_{A F}=3 \mathrm{~nm}$ sample, the curves do track together quite well, indicating that couping at top and bottom AF interfaces are on a par. Also, the thin layers in general show rather little variability in exchange energy at low temperature. The thick layers, on the other hand, display considerable change across samples, as seen in the figure. The exchange for the $t_{A F}=1 \mathrm{~nm}$ sample is very low, then it shoots up at $3 \mathrm{~nm}$, dips again at $5 \mathrm{~nm}$, and gradually rises after that, with the gap between thin and thick layers appearing to narrow with increasing AF thickness.

Where blocking temperature is concerned, the results are decidedly unambiguous. We observe a sequence of blocking temperatures in the $H_{E B}(T)$ curve that go from low to high as $t_{A F}$ is increased. Additionally, the sensitivity of $T_{B}$ diminishes with 


\begin{tabular}{c|cc}
\hline \hline$t_{A F}(\mathrm{~nm})$ & $m_{\text {bottom }} / m_{\text {top }}$ & $d_{\text {bottom }} / d_{\text {top }}$ \\
\hline 3 & 3.61 & 3.70 \\
5 & 3.44 & 3.81 \\
7 & 3.65 & 3.55 \\
10 & 3.65 & 3.71 \\
\hline \hline
\end{tabular}

Table 3.2: Ratios of the total moments of the bottom vs. top layers and the corresponding ratios of their thicknesses determined from x-rays.

increasing $t_{A F}$. For the AF thickness range of $t_{A F}<5 \mathrm{~nm}$ in particular $T_{B}$ is highly sensitive, but in the larger range we see values of $T_{B}$ that seem to asymptotically approach the bulk Néel temperature of about $78 \mathrm{~K}$.

The blocking temperatures determined from hysteresis loops are fairly coarse estimates, as the measurements are taken at $5 \mathrm{~K}$ intervals. We believe that an alternate approach to gauging the $T_{B}$ distribution is offered by examination of TRM. In the absence of EB the TRM of an FM film should reveal information about its magnetic anisotropy. As thermal energy progressively weakens the anisotropy we should see a monotonic decrease in TRM that drops to zero at the Curie temperature of the FM. However, in the case of Co, the Curie temperature is so much higher $\left(T_{C}\right.$ $=1388 \mathrm{~K})$ than the temperature region of interest $(T<100 \mathrm{~K})$ that this component of the TRM is practically linear. In our exchange biased systems we see a secondary decay in the the TRM which is associated with the rightward shift of the loop as $H_{E B}$ decays. A look at the hysteresis loops shown in Figure 3.5 reveals that there is no readily visible change in the anisotropy of the loops with increasing temperature, which would reveal itself in an increasing shear of the loops. Even though we are not measuring in a field, it is in effect as though we are still sampling a portion of the hysteresis loop. When the blocking temperature is reached, the decay in TRM due to the rightward shift of the magnetization curve will stop, and only the decay from increasing anisotropy will remain. Therefore $T_{B}$ ought to be approximately 
coincident with an inflection point in the TRM curve.

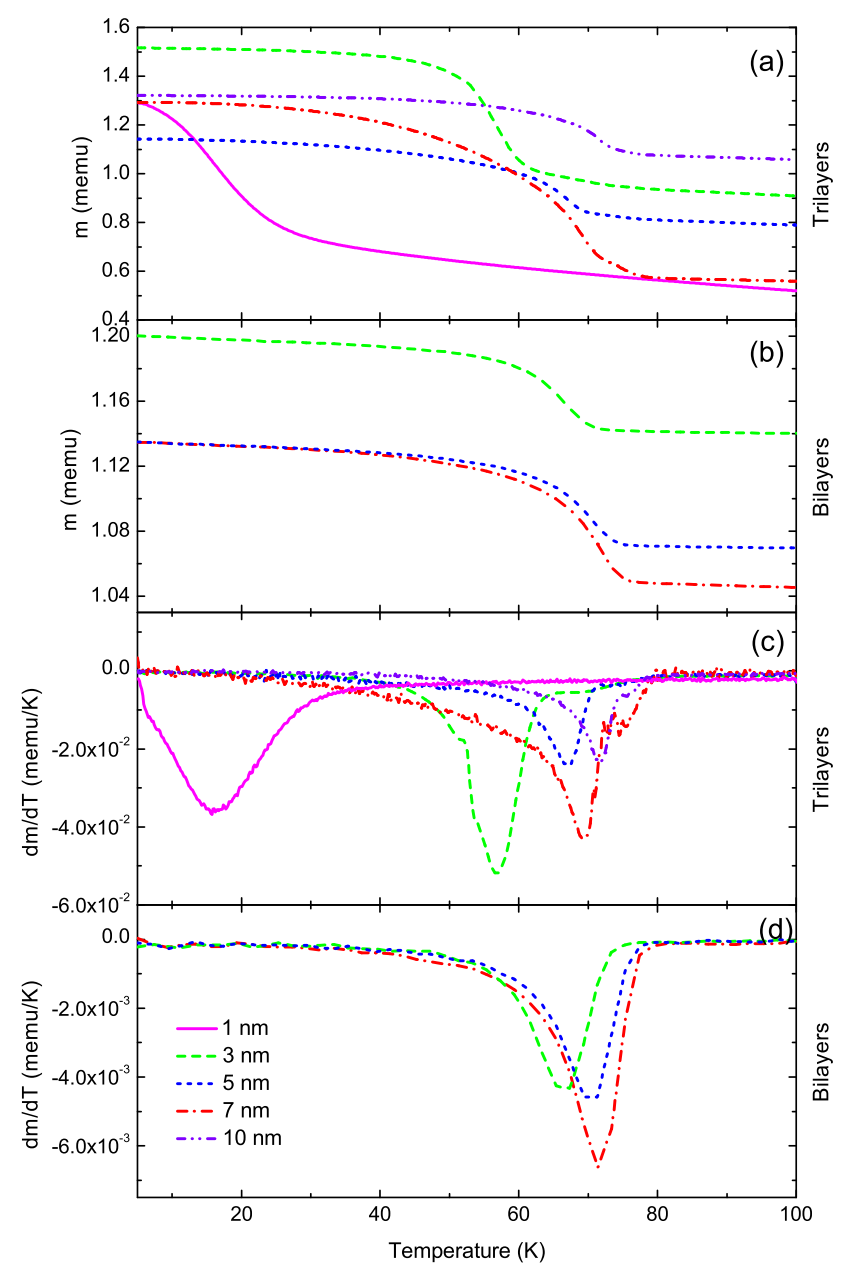

Figure 3.9: TRM vs. temperature curves for (a) trilayers and (b) corresponding bilayers. Peaks in the first derivative of TRM data for (c) trilayers and (d) bilayers show magnetic transitions correlating well with the $T_{B}$ obtained from hysteresis loops. See Figure 3.10.

In Figure 3.9, we see TRM curves for each of the samples together with their first derivatives. Each curve shows exactly the kind of behavior described above. The inflection point in each TRM curve coincides with a peak in the derivative which we take to be indicative of $T_{B}$. Values of $T_{B}$ obtained from the TRM data are plotted together with those taken directly from the $H_{E B}(T)$ curve in the figure, showing a 
good overall match.

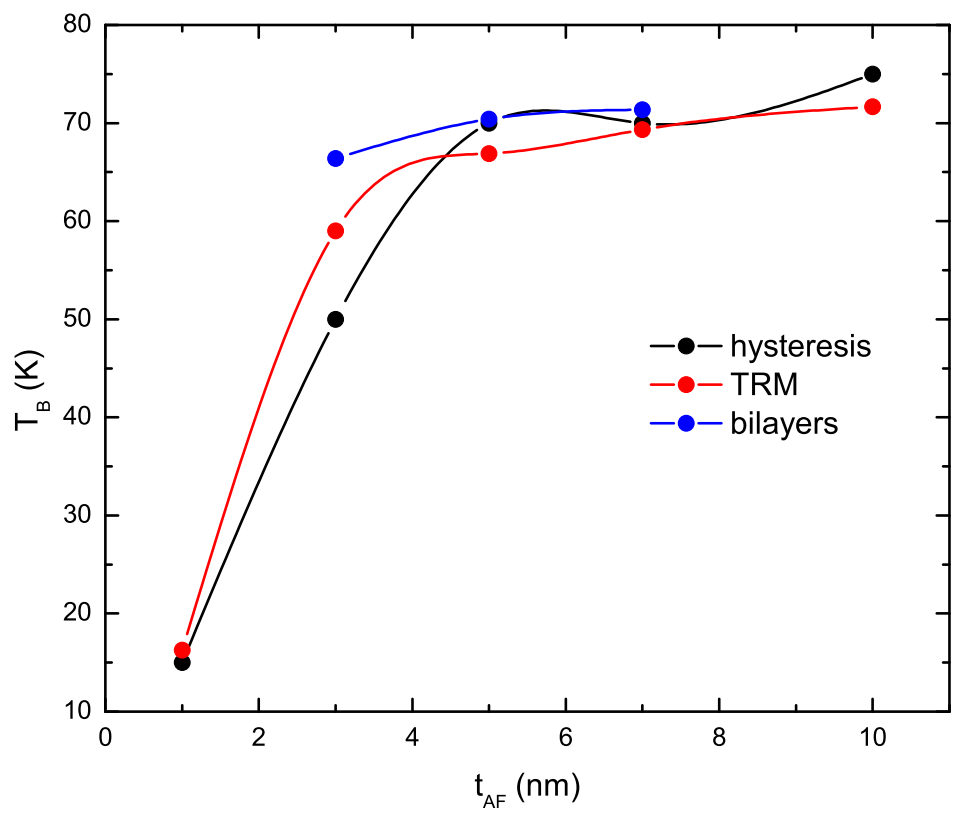

Figure 3.10: Blocking temperatures for trilayers obtained from hysteresis loops and TRM, as well as blocking temperatures of bilayer samples from TRM.

The bilayer samples display generally more pronounced behaviors than the thick Co layers in the trilayer samples. We see larger coercivity, exchange bias, exchange coupling, and blocking temperatures in bilayers. The coercivity, shown in figure 3.11 for $5 \mathrm{~K}$, displays little sensitivity to AF thickness. An example of the bilayer hysteresis measurements is shown in the figure 3.12. Note that compared to loops in figure 3.5 the bilayer displays stronger anisotropy as revealed by the relative lack of shear in the loops. This is also apparent in the TRM data, where the bilayers have a smaller drop in moment as they transition across the blocking temperature. Figure 3.13 shows a direct comparison of the $t_{A F}=7 \mathrm{~nm}$ trilayer and bilayer hysteresis above the blocking temperature. If the difference in anisotropy were the result of differences 
in interfacial magnetic ordering in the FM induced by exchange anisotropy, then we would expect such a difference to disappear at temperatures above $T_{B}$. Figure 3.13 suggests that the weakening anisotropy of trilayers has an independent mechanism from EB and results from direct interaction between the FM layers. The likely source of this effect is Néel coupling or so-called "orange peel" coupling that is due to the formation of short range dipole fields arising from interface roughness. This type of coupling is commonly observed in systems that feature FM layers with non-metallic spacers. The magnitude of the coupling is expected to drop off exponentially with spacer thickness [66]. As the roughness is relatively homogeneous across samples, we would anticipate weaker FM anisotropy in samples with low $t_{A F}$. If we take the anisotropy to be fairly represented by the relative drop seen in the TRM seen in figures 3.9 (a) and (b), then there is a good overall agreement with this expectation. The exception is the $t_{A F}=7 \mathrm{~nm}$ sample, which appears to have a weaker anisotropy than the $5 \mathrm{~nm}$ one. This could also contribute to the relatively higher coercivity observed in bilayers. Without inter-layer FM coupling, they have stronger anisotropy and correspondingly require higher fields to switch.

Néel coupling may explain another feature of our data, namely the indistinguishability of thick and thin FM layers in the $t_{A F}=1 \mathrm{~nm}$ sample. When the roughness oscillations across the spacer are in phase with one another, the Néel coupling is effectively ferromagnetic [67]. In the $1 \mathrm{~nm}$ sample, we still observe exchange bias, which we would expect to be quite distinct for the top and bottom Co layers. However, it is likely the case that the Néel coupling is so strong in this sample that the layers cannot switch independently, and the hysteresis is dominated by the switching characteristics of the thick layer.

While the exchange energy for the bilayer samples is consistently larger than the 


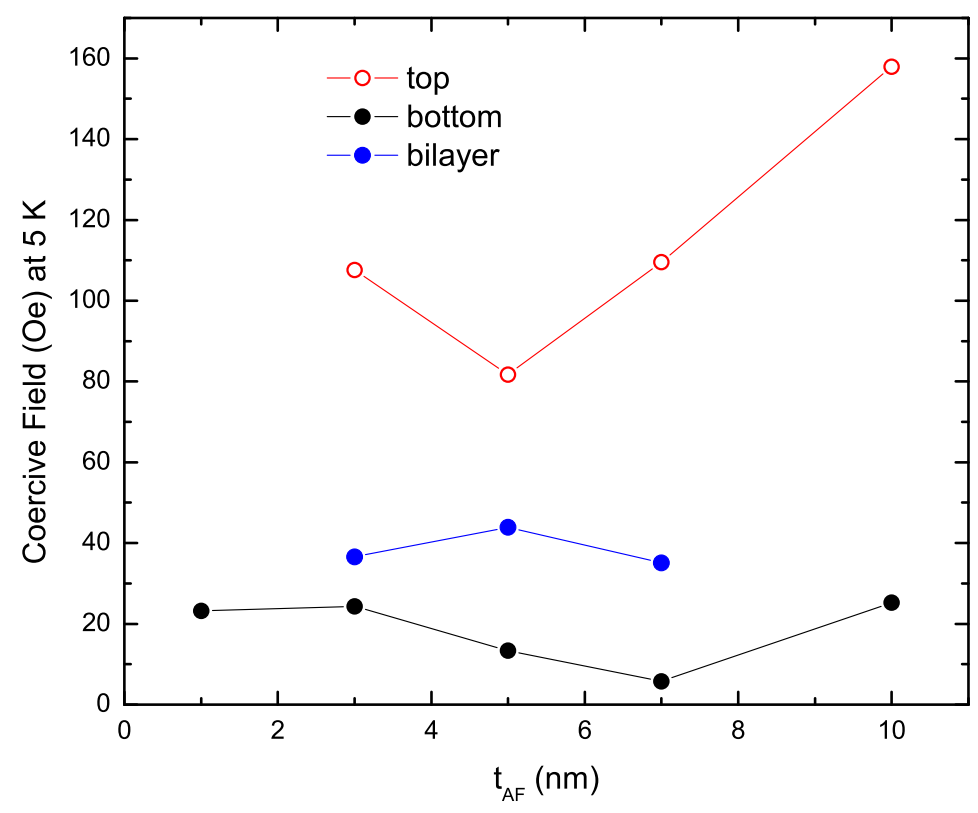

Figure 3.11: Coercivity vs. AF thickness for top and bottom FM layers, as well as in corresponding bilayers.

corresponding trilayers, there is less variability among them. The $t_{A F}=3$ and $5 \mathrm{~nm}$ samples are almost the same, with a marginally higher average value in the $3 \mathrm{~nm}$ one in the region well below $T_{B}$. The energy of the $7 \mathrm{~nm}$ sample shows about a $17 \%$ drop from the $5 \mathrm{~nm}$ sample. As only three bilayer samples were grown, it is only speculative how they would behave for thicker values of $t_{A F}$, but the data obtained are not inconsistent with the supposition that the discrepancy in behavior between bilayers and trilayers would vanish for some sufficiently thick AF sample.

Blocking temperatures for the bilayers, obtained from TRM (shown in Figure 3.10), are also a little higher than in the case of the trilayers, but the difference is not pronounced, especially for the $t_{A F}=5$ and $7 \mathrm{~nm}$ samples. The trend is nonetheless clear that $T_{B}$ shows an increase with AF thickness. It is commonly observed that 


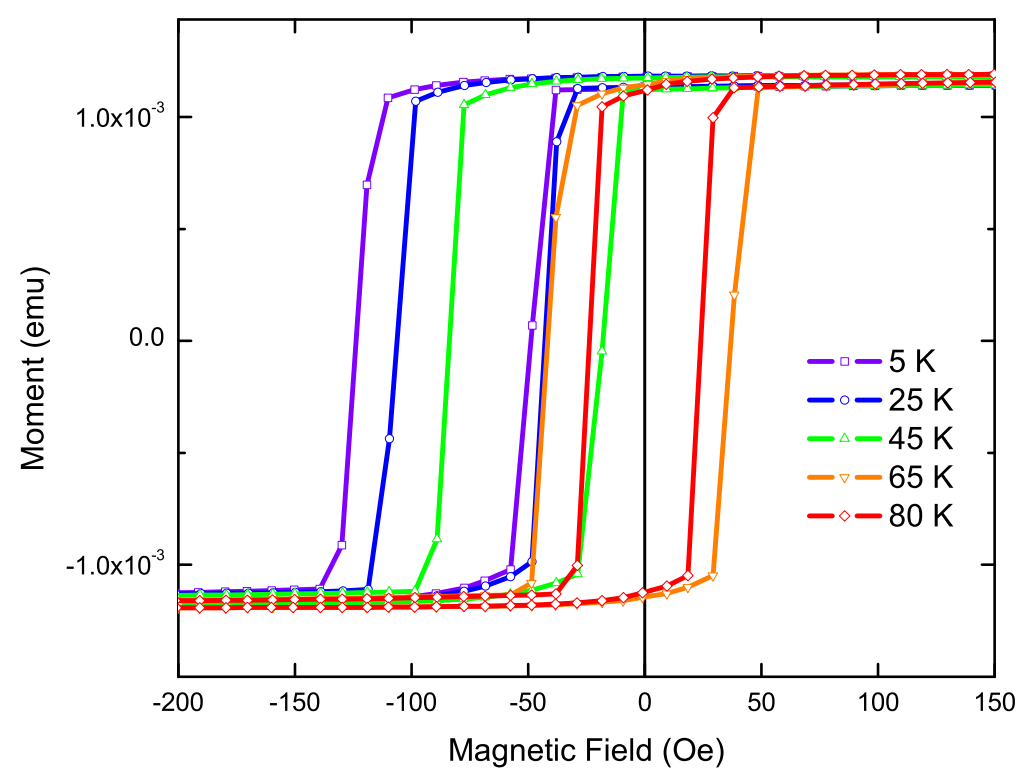

Figure 3.12: Hysteresis loops taken on the $t_{A F}=3 \mathrm{~nm}$ bilayer sample as a function of temperature.

blocking temperature is coupled to AF grain size through finite size effects [11]. In our study, the lateral dimensions of the AF grains are unconstrained, but likely much larger than the film thicknesses studied. Therefore, the crucial limiting factor in grain size is the thickness of the deposited film, with thinner films having smaller grains.

Measurements of the hysteretic character of anisotropic magnetoresistance (AMR) provide an alternative means of examining the magnetization reversal. AMR data for each of the patterned trilayer samples is shown in Figure 3.14, normalized relative to its value at saturation. The magnetic reversal is indicated by the minima in the resistance vs. field curve as the moments rotate perpendicular to the applied current. The exchange bias determined by AMR matches, within the relatively larger error of the AMR, to those values obtained from magnetometry. However, as shown in Figure 3.15, the coercivity obtained via AMR is considerably larger for all samples than 


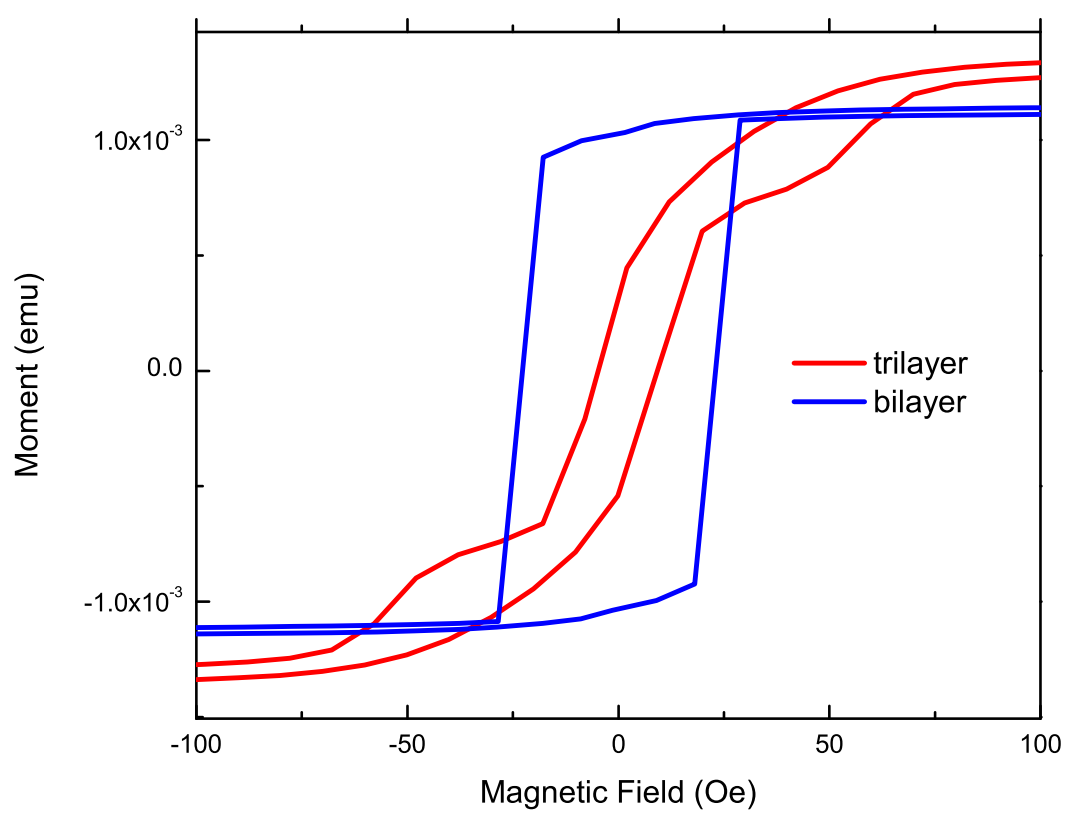

Figure 3.13: Hysteresis curves for bilayer and trilayer samples with $t_{A F}=7 \mathrm{~nm}$, taken above the blocking temperature at $80 \mathrm{~K}$.

those taken from magnetometry at the comparable temperature of $15 \mathrm{~K}$. An original source of concern was that the switching characteristic was altered by a change in shape anisotropy, resulting from patterning of the Hall bar. Accordingly, magnetometry was re-measured for one of the patterned trilayers. The resulting hysteresis curve showed a smaller signal because the effective sample size was reduced, but the shape of the curves were otherwise identical.

This discrepancy can be resolved by the observation that AMR and magnetometry are sensitive to different switching modes. The magnetization of an FM film can reverse in two distinct modes, namely by the nucleation and expansion of reversed domains (domain wall motion) or coherent rotation of magnetization within domains (domain rotation). In the former mode, the moments that undergo rotation out of the 


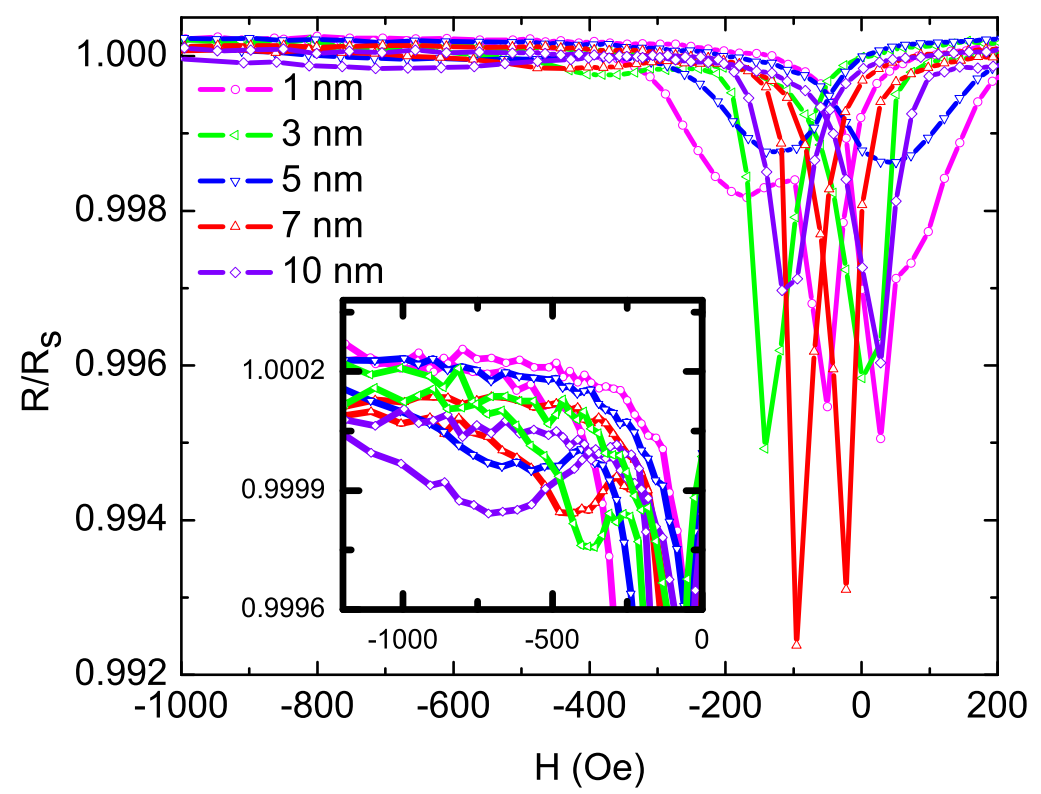

Figure 3.14: Magnetoresistance $R$ of trilayers normalized against resistance at saturation $R_{S}$, taken at roughly $14 \mathrm{~K}$.

field plane are only those small minority located within the domain wall. Hence AMR is insensitive to domain wall motion. The magnetometry picks up the component of the total moment that is parallel to the field axis. Therefore, it does not discriminate between the microscopic reversal mode. Similar discrepancies were observed in EB bilayers in reference [68]. In Figure 3.16, the hysteresis loop for the $t_{A F}=10 \mathrm{~nm}$ sample at $15 \mathrm{~K}$ is shown together with the switching fields obtained via AMR at comparable temperature. By the time the AMR shows switching, most of the net moment has already reversed. It may be noted that there is an elongated tail in the hysteresis, highlighted in the figure, which shows that there is a minority population of domains in the FM film that are strongly resistant to reversal. Therefore, we conclude that the dominant mode of magnetization reversal in our samples is by the domain wall motion mechanism. Meanwhile, there are at least some portions of the 


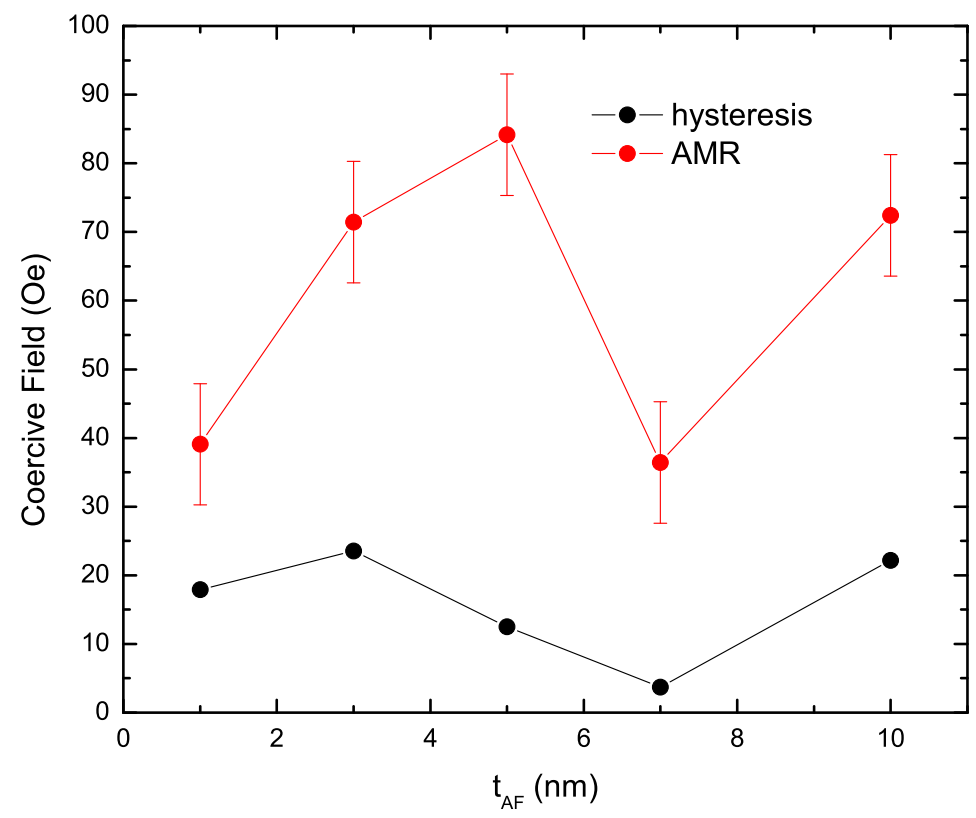

Figure 3.15: Low temperature coercivity vs. AF thickness for trilayers obtained from hysteresis loops and AMR.

FM that undergo coherent rotation at higher applied fields. A possible explanation for this is that defects might pin the domain walls and inhibit their further motion until they are forced to rotate at higher fields.

Another interesting observation from the AMR data concerns the $t_{A F}=1 \mathrm{~nm}$ sample. In addition to the sharp dips in resistance, there are two broad peaks that show larger coercivity and EB. All of the samples show these secondary peaks, which we attribute to the reversal of the thin layer. However, for the thinnest AF sample these peaks are particularly prominent. In the hysteresis curves there was no detectable distinction between the reversal of the two layers, but they are clearly distinguishable in the AMR data.

While we have not systematically studied the effects of different cooling fields 


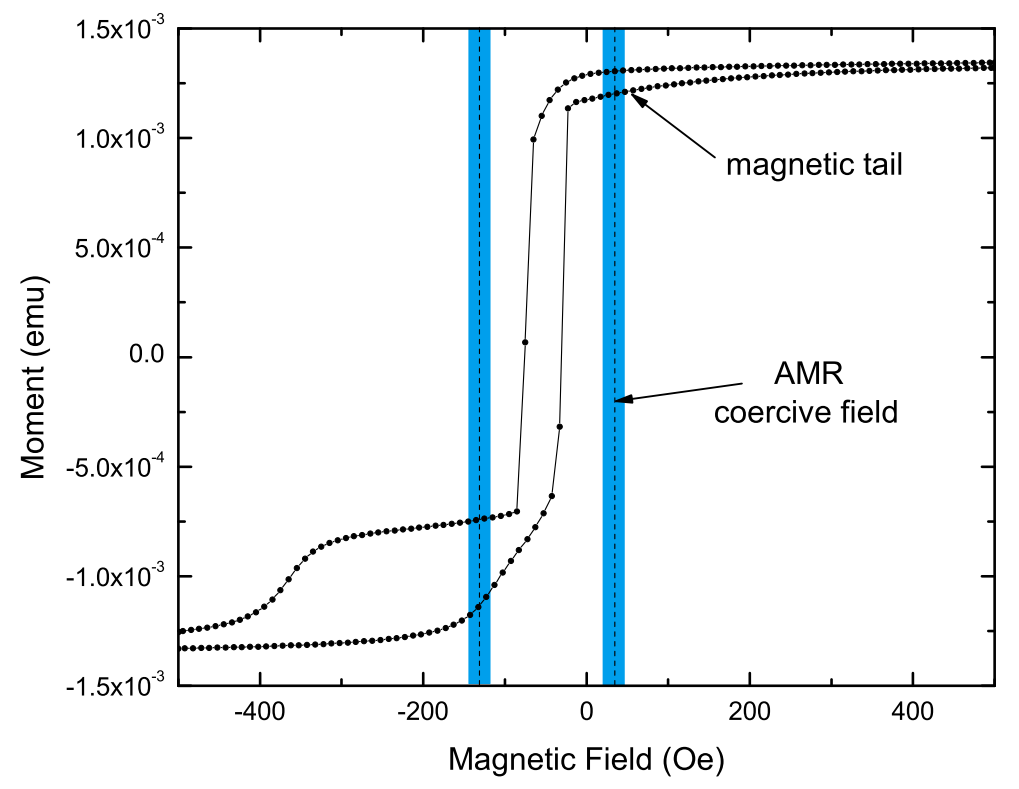

Figure 3.16: Hysteresis loop of $t_{A F}=10 \mathrm{~nm}$ sample at $15 \mathrm{~K}$ with the coercive field obtained from AMR highlighted. The AMR reversal overlaps with the elongated tail of minority moments that reverse by coherent rotation.

on our system, there are some observations worth noting. Other studies of the $\mathrm{Co} / \mathrm{FeF}_{2}$ interface have found that the sign of $\mathrm{EB}$ can be switched by the application of a sufficiently high cooling field [69]. The conclusion being that the Fe and Co moments couple antiferromagnetically. Under high fields the Fe moments would be pulled into the same direction as the Co, giving rise to an unstable exchange coupling at the interface. In one of our samples, we field cooled in $90 \mathrm{kOe}$ down to $5 \mathrm{~K}$ in an attempt to produce positive EB, however, no such reversal was observed. The superficial appearance of EB reversal for the thick FM layer could be induced in the $t_{A F}=7$ and $10 \mathrm{~nm}$ samples only, by the application of a weak cooling field of about -25 Oe. In these two samples, the coercivities of the thin and thick layers are slightly distinct above the Néel temperature. By cooling the sample in a configuration in 
which the thick layer has reversed, but the thin one has not, it is possible to bias the thick layer in the positive direction, as shown in Figure 3.17. However, it should be stressed that this is not a true EB reversal, as the sign of EB is defined relative to the cooling field. While the field is negative, it is effectively positive for the thin layer because it is still magnetized in the positive direction.

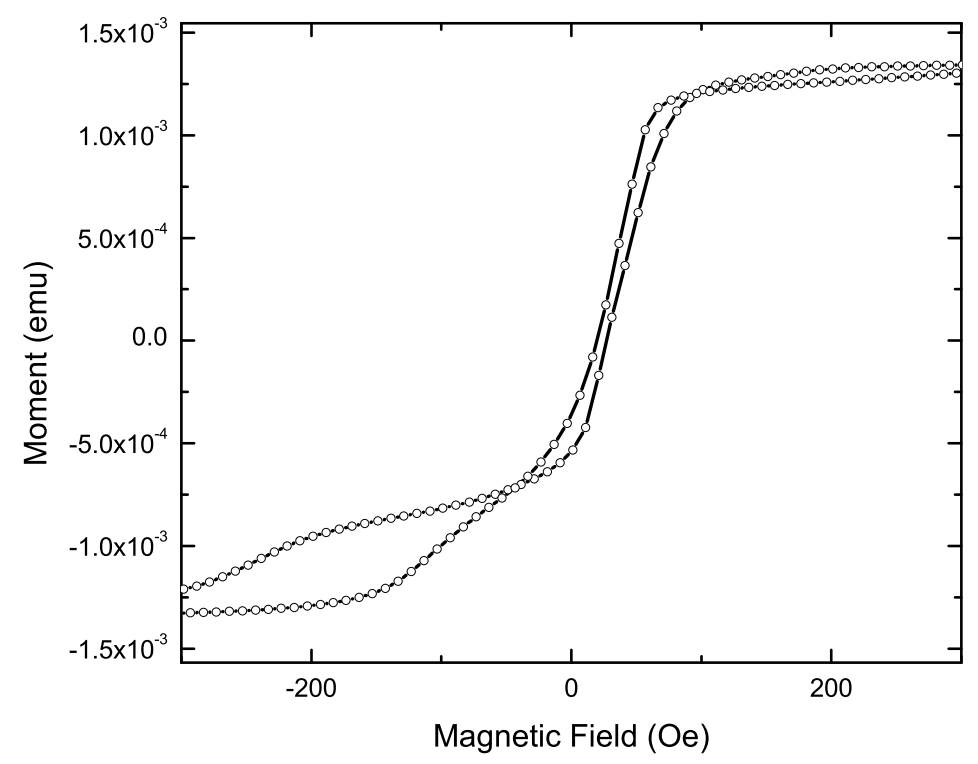

Figure 3.17: Hysteresis loop of $t_{A F}=7 \mathrm{~nm}$ sample taken after field cooling in -25 Oe, after sweeping the field from positive saturation. The soft, bottom FM layer has the appearance of being positively biased by the weakly negative field.

A look at the low temperature variation of the exchange energy with $t_{A F}$, shown in figure 3.18, reveals two interesting observations that bear further analysis. For one, the exchange energy of bilayers is consistently higher than that of either interface in trilayers. Also, the exchange energy of the bottom layer experiences a pronounced fluctuation with AF thickness. The top layer displays similar behavior, but to a smaller degree. More specifically, the energy rises considerably as $t_{A F}$ goes from 1 to $3 \mathrm{~nm}$, and then drops back down at $T_{A F}=5 \mathrm{~nm}$, before gradually rising with 
increasing thickness. In the following, we will attempt to explain these observations by appealing to known models of EB.

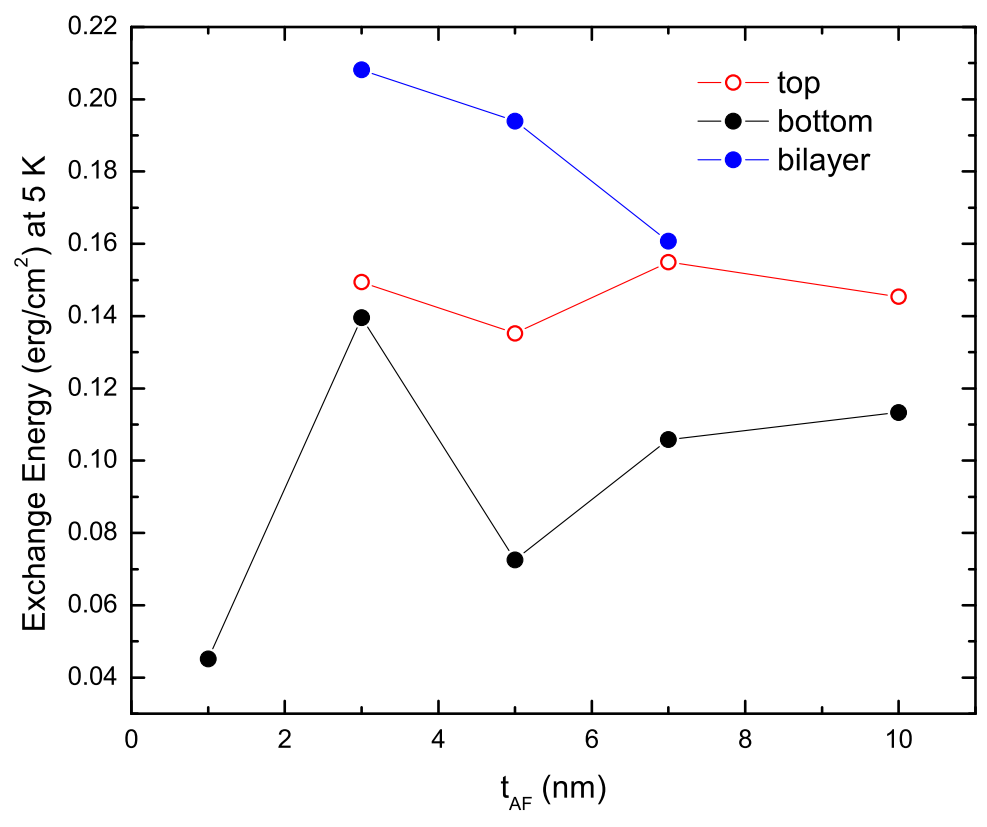

Figure 3.18: Interface exchange energy vs. $\mathrm{AF}$ thickness at $\mathrm{T}=5 \mathrm{~K}$.

First, consider a general thought experiment. Suppose we have a trilayer structure in which the interfacial exchange coupling can be selectively turned on and off independently at the two interfaces. In effect, we can alternate between individual bilayer structures without changing any other intrinsic parameters of the system. If the system is field-cooled with one interface active, the AF seeks out a configuration that minimizes its net energy. This structure, whatever its microscopic details might be, must extend over at least some distance into the bulk AF because the surface spins are exchange coupled to spins deeper in the film. Likewise, if the same procedure is followed with the opposite interface active instead, some other structure will arise. Assuming that these two structures have a region of overlap in the bulk, 
i.e. some spins in the bulk exchange couple to both surfaces, then it follows that the two interfaces will compete with one another, when both active, to determine the optimal cooling configuration. The result would tend to be a compromise structure that would allow neither interface to couple to as many pinned, uncompensated moments as in a bilayer, where there is no competition. Khan et al. [70] employed essentially this argument to explain the diminished $\mathrm{EB}$ in $\mathrm{Co} / \mathrm{Ni} / \mathrm{Ni}_{25} \mathrm{MN}_{75} / \mathrm{Ni} / \mathrm{Co}$ $\mathrm{FM} / \mathrm{AF} / \mathrm{FM}$ structures relative to corresponding bilayers.

If we assume that there is a critical length scale over which the interface influences the bulk, then it is clear that the difference in EB between bilayers and trilayers ought to disappear beyond that length scale. In our results, we still see a significant difference in exchange energy between the $t_{A F}=7 \mathrm{~nm}$ trilayer and bilayer samples. Therefore, it is not clear what is the maximum distance, over which the bulk is affected by the interface, but it is likely well beyond what we have probed. By field cooling $\mathrm{Ni}(50 \mathrm{~nm}) / \mathrm{FeF}_{2}(200 \mathrm{~nm}) / \mathrm{FeNi}(50 \mathrm{~nm})$ trilayers with the interfacial magnetizations in parallel and anti-parallel configurations, Morales et al. [71] observed that the bulk ordering of the AF influenced EB over a much larger thickness than in the samples we have studied.

In our trilayer samples, that the exchange anisotropy experiences something like a resonance at $t_{A F}=3 \mathrm{~nm}$ as seen in figure 3.18. Data taken from a second, nominally $3 \mathrm{~nm}$ thick sample showed qualitatively similar behavior. It is often observed that $\mathrm{EB}$ is very weak or unobservable in bilayers with a very thin AF layer [11]. Above some critical AF thickness, the EB dramatically increases and then typically stabilizes with increasing thickness. In our case, we also see a dramatic increase in EB from 1 to $3 \mathrm{~nm}$, but it then drops back down considerably. Similar behavior has been observed elsewhere, but different researchers have interpreted this in terms of 
mechanisms particular to their system. Castro et al. [72] observed a peak in $H_{E B}$ at AF thicknesses of 6 and $8 \mathrm{~nm}$ for $\mathrm{FeNi} / \mathrm{IrMn} / \mathrm{Co}$ trilayers grown on seed layers of $\mathrm{Cu}$ and $\mathrm{Ta}$, respectively. They interpreted this to result from lack of stress in the IrMn that increases its magnetocrysalline anisotropy. In their study of trilayers based on $\mathrm{LaSrMnO}_{3}$ and $\mathrm{SrMnO}_{3}$, Jungbauer et al. [73] observed maximum EB at $t_{A F}=12$ $\mathrm{nm}$, which they also attributed to strain in the AF, which caused the formation of a spin glass state at the interface. Similar behavior has also been observed in the $\mathrm{FeNi} / \mathrm{FeMn} / \mathrm{FeNi}$ system $[63,74]$. However, this pattern is not common to all trilayer systems that have been studied [75-77], therefore it is not clear if our results are due to some peculiarity of our system or if there is some common underlying feature with the above mentioned studies.

A necessary condition for EB to occur is that the interface anisotropy energy of the AF must be greater than the exchange energy, i.e. $K_{A F} t_{A F} \geq J_{\text {int }}$ [11]. If this condition is not met then there will be insufficient anisotropy to pin the AF moments in place, and they will reverse with the FM spins. The very weak EB observed for $t_{A F}=1 \mathrm{~nm}$ is likely due to this.

In order to explain the thickness dependent behavior of the exchange energy above $1 \mathrm{~nm}$, we will take a closer look at the random field model developed by Malozemoff [20]. In this approach, surface roughness (or other defects) generate a random field of local exchange energies over the interface with a uniformly magnetized FM. Averaged over the entire surface, the random exchange energies would average out to zero, yielding zero EB. Malozemoff suggested that it would be energetically favorable for these local surface regions to form domains with domain walls perpendicular to the surface. Each domain could then adopt an AF order that minimizes its exchange energy during field cooling. Domains that remain pinned under FM magnetization 
reversal would then exert a torque on the FM spins, producing EB. The average interfacial exchange energy over a surface domain would tend to decrease with a larger area. More precisely,

$$
\sigma \sim \sigma_{l} / \sqrt{N}
$$

where the interfacial exchange energy $\sigma$ is on the order of the local exchange energy $\sigma_{l}$, and $N$ is the number of interfacial spins in the domain. This would result in a tendency to form smaller domains in order to maximize exchange energy. However, smaller domain walls have higher anisotropy energy associated with their large curvature. The balancing of these energies leads to a domain size $L$ on the order of the thickness of a domain wall $\pi \sqrt{A / K}$, where $A$ is the exchange stiffness and $K$ the magnetocrystalline anisotropy of the $\mathrm{AF}$, respectively. Malozemoff envisioned these domain walls forming hemispherical bubble-like shapes at the interface, as shown in figure 3.19 , in order to minimize the anisotropy energy over the domain surface.

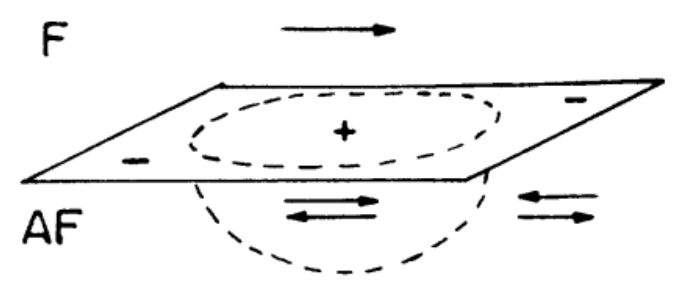

Figure 3.19: Schematic representation of AF domains with global spin ordering represented by arrows [20].

If we apply Malozemoff's picture to a large $t_{A F}$ sample, we should see a structure like the one pictured in figure 3.20(a). The random field at each interface would produce a "sea of bubbles" [20] that ought to effectively decouple the two interfaces, leading both interfaces to behave like independent bilayers. As $t_{A F}$, and accordingly the distance between the domain walls is decreased, the domains would interact with one another via the bulk AF separating them. This would become especially strong 
as the space between bubbles approaches the length of a domain wall. Since the interfacial area of the domain is governed by a balance between exchange energy and domain wall anisotropy, this might lead to a broadening of the interface and a consequent decrease in exchange energy with decreasing $t_{A F}$. In order for a trilayer to form bubble domains on both interfaces, there must be a minimum AF thickness, on the order of several domain walls in extent, as shown in figure 3.20(b).

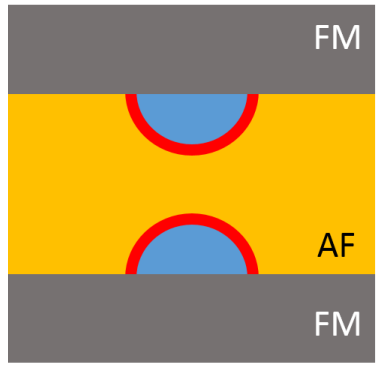

(a)

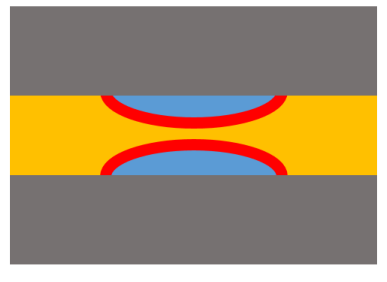

(b)

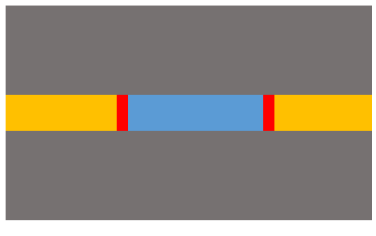

(c)

Figure 3.20: Diagram of three different domain wall structures in trilayers with different AF layer thicknesses. (a). Hemispherical bubble shaped domains near the $\mathrm{AF} / \mathrm{FM}$ surfaces in a thick $t_{A F}$ sample. The yellow and blue regions of the AF represent areas with alternate global spin ordering, while domain walls are outlined in red. (b). Deformed bubble shaped surface domains in close proximity to one another. (c). Vertical domain walls cutting across the AF film.

One possible explanation, for the relatively strong exchange energy of the $t_{A F}=$ $3 \mathrm{~nm}$ sample could lay in the relative ease of perpendicular domain wall formation in a thin AF. For a sufficiently thin AF (too thin for lateral domain wall formation), perpendicular domain walls might still be formed which cut through the entire film from interface to interface. This could be justified within the framework of the random field model by supposing that there are local exchange energies $\sigma_{l 1}$ and $\sigma_{l 2}$ on opposite sides of the AF. The average interfacial exchange energy for a domain 
with two interfaces would therefore go as

$$
\sigma \sim \frac{\sigma_{l 1}+\sigma_{l 2}}{2 \sqrt{2 N}}
$$

This is formally the same as the single interface random field model, but with an exchange energy that scales differently with lateral domain size. The energy cost of forming a vertical domain wall across the film is proportional to the film thickness. Therefore, the formation of such domains would be more favored for thinner films. If the thickness of the AF is increased, we would expect the energy balance between the domain wall formation and exchange energy to result in weaker EB. We have seen from the SLD profile of this sample that the bulk is interdiffused with the interfaces. Defects associated with this interdiffusion could act as preferred locations for the formation of domain walls in the AF that help to lower the overall energy of the system [78]. The energy associated with forming a perpendicular domain wall might be especially favorable in the interdiffused region of a film compared to the non-interdiffused region.

The trend observed in the exchange energy vs. AF thickness could be explained as a combination of the two modes of domain formation outlined above, based on Malozemoff's model. The relatively low EB observed in the $t_{A F}=5 \mathrm{~nm}$ trilayer may be at a cross-over point between these modes. That is, this sample may have been too thick to effectively form vertical domain walls, but too thin to form lateral domain walls that can decouple the two surfaces. The estimated domain wall width in $\mathrm{FeF}_{2}$ is on the order of $1 \mathrm{~nm}$ [79], therefore the scale for the formation of separate surface domains on the two interfaces would be close to the thickness of this sample.

Our data unambiguously confirm that EB is not a purely interfacial effect. However, the range of its propagation remains unclear. The same complexities of real 
world systems, such as interface roughness, interdiffusion, crystallinity, and grain size, that have made EB such an enduringly elusive phenomenon to quantitatively understand are present in trilayer systems as well. Our conceptual model provides a framework within which to think about these systems, but a more rigorous treatment of how it is manifested in real structures will require further investigation. 


\section{Chapter 4}

\section{Multiferroic $\mathrm{BaMF}_{4}$}

\subsection{Introduction}

The isostructural family of compounds $\mathrm{Ba} M \mathrm{~F}_{4}$, with $M$ being a transition metal or Mg, first attracted interest in the late 1960's [80-89] for potential piezoelectric applications [87]. More recently, renewed interest has been generated by its multiferroic properties, specifically the simultaneous presence of long range antiferromagnetic and ferroelectric long range ordering in several members of the family, and the possibility of magneto-electric coupling [90-92]. Here, results are presented from the first time synthesis, in thin film form, of the compounds for which $M=\mathrm{Fe}$, Co, and $\mathrm{Ni}$, together with analysis of their structural, magnetic, and ferroelectric properties.

$\mathrm{Ba}_{\mathrm{MF}}$ compounds crystallize in an orthorhombic structure belonging to the space group Cmc2 $2_{1}$ [81]. A model unit cell of this structure is pictured in figure 4.1. The bulk structure consists of distinct sheets of octahedrally surrounded di-valent transition metal ions. The octahedra form linear chains along the $c$-axis and pucker at their shared corners into a zig-zag shape along the $a$-axis as seen in figure 4.2. Barium ions nest in the spaces between these octahedra. Neighboring sheets are staggered 
relative to one another by half a lattice parameter along the $c$ direction. $B a M F_{4}$ is an example of a "geometric ferroelectric", [90] i.e., the mechanism of ferroelectric switching is due to a structural transformation of the lattice without significant inter-ionic charge transfer, as is the case for the more widely studied ferroelectric oxide perovskites such as $\mathrm{PbZr}_{x} \mathrm{Ti}_{1-x} \mathrm{O}_{3}$. A model of the structure viewed in the $a b$-plane is shown in figure 4.2 in its two stable polarization states along the $a$ axis. Measurements of the bulk ferroelectric saturation polarization conducted by Eibschütz et al. [84] found values of $P_{s}=8.0 \mu \mathrm{C} / \mathrm{cm}^{2}$ and $6.7 \mu \mathrm{C} / \mathrm{cm}^{2}$ for $\mathrm{BaCoF}_{4}$ and $\mathrm{BaNiF}_{4}$ respectively, but no switching was observed for $\mathrm{BaFeF}_{4}$, ostensibly on account of overly high leakage currents. A theoretical analysis by Ederer and Spaldin [90] has suggested that switching in the Fe compound is probably realizable, although the energy barrier between polarizations states should be higher, requiring a stronger switching electric field than for the other compounds.

All of the $\mathrm{BaMF}_{4}$ fluoride compounds are antiferromagnetic with the three under consideration here having Néel temperatures at approximately 70 K [92]. The magnetic lattice structure has a unit cell twice as large as the conventional unit cell, as depicted in figure 4.3. As the separation between planes is relatively large, the transition metal ions have stronger exchange interaction within each plane than between them. This weak interplanar coupling gives $\mathrm{BaMF}_{4}$ a largely two-dimensional antiferromagnetic behavior. In $\mathrm{BaFeF}_{4}$ and $\mathrm{BaNiF}_{4}$ the spins are parallel to the $b$-axis with $\mathrm{BaNiF}_{4}$ also having a weak secondary antiferromagnetic order along the $a$-axis produced by the Dzialoshinskii-Moriya interaction, and manifesting itself as a slight $1^{\circ}-3^{\circ}$ canting along the $a$ direction [91]. In the case of $\mathrm{BaCoF}_{4}$, the easy axis is along the direction of the $a$ lattice parameter. While the intraplanar and interplanar couplings are both antiferromagnetic, $\mathrm{Ba} M \mathrm{~F}_{4}$ can exist in two magnetic phases [86]. 


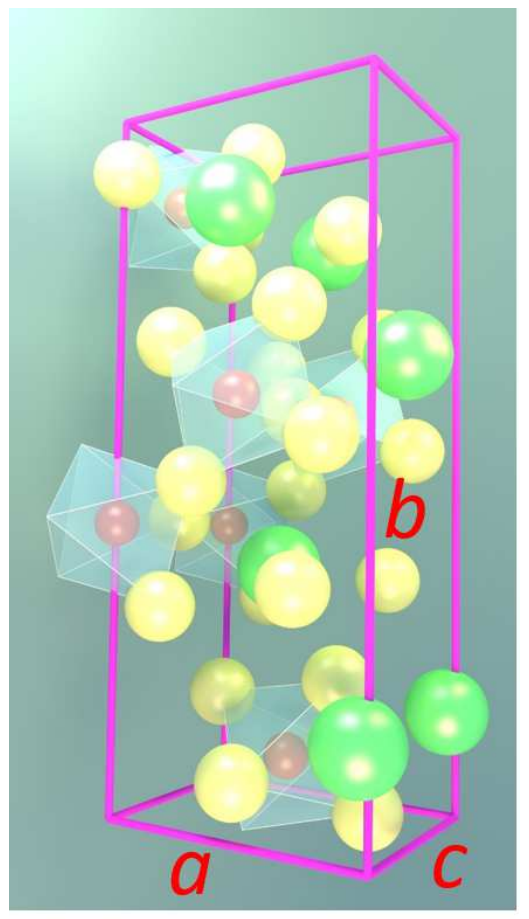

Figure 4.1: A model of unit cell of $\mathrm{BaMF}_{4} \cdot \mathrm{Ba}^{2+}$ ions are pictured in green, $\mathrm{Co}^{2+}$ are in red and surrounded by octahedra of $\mathrm{F}^{-}$ions in yellow.

In phase $A$ the coupling between next nearest neighboring planes is antiferromagnetic while in phase $B$ it is ferromagnetic. These two phases have nearly the same energy and simultaneously coexist within the same sample.

\subsection{Experimental Procedures}

\subsubsection{Growth}

Three variant substrates were utilized in this work. First, pre-diced $(5 \mathrm{~mm} \times$ $10 \mathrm{~mm}$ ), single-side polished $\mathrm{Al}_{2} \mathrm{O}_{3}$ (0001) substrates were prepared by a procedure consisting of degreasing in an ultrasonic bath of acetone (10 minutes) and methanol (10 minutes) prior to annealing at $1200{ }^{\circ} \mathrm{C}$ for 2 hours to reconstruct the surface 


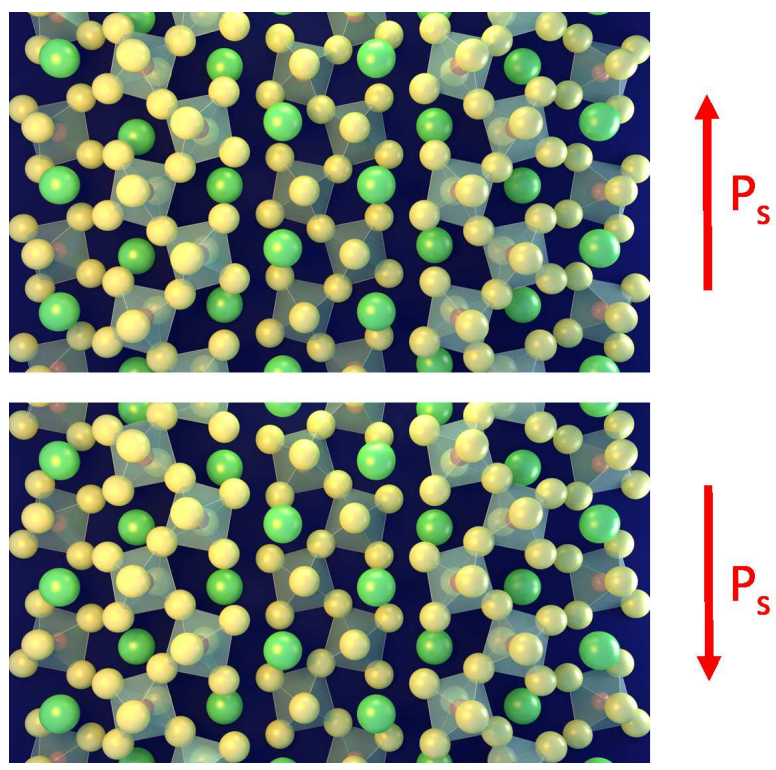

Figure 4.2: A model of the $\mathrm{Ba}_{\mathrm{MF}}$ lattice with the ferroelectric $a$ direction along the vertical and the long $b$-axis on the horizontal. The top and bottom pictures show the structural configuration of the lattice with oppositely polarized states with polarization $P_{s}$. Switching occurs by a combination of octahedral rotation and displacement of the $\mathrm{Ba}$ ions (green).

and remove surface contamination [93]. Some of these substrates were used directly as a growth surface while others underwent further preparation. In one variation, an epitaxial film of (111) Pt was deposited on the $\mathrm{Al}_{2} \mathrm{O}_{3}$ by $\mathrm{DC}$ magnetron sputtering. In order to achieve epitaxy, these films had to be grown at a temperature of $580{ }^{\circ} \mathrm{C}$. After allowing the substrates to reach equilibrium temperature, the $\mathrm{Pt}$ was sputtered in an Ar atmosphere at a pressure of $3 \mathrm{mT}$, and a constant current of $50 \mathrm{~mA}$. Pt was deposited at a rate of about $2 \mathrm{~nm} / \mathrm{min}$ to a thickness of $50 \mathrm{~nm}$. In another variation, interdigitated Pt electrodes were deposited on prepared sapphire substrates. A photoresist mask consisting of six pairs of electrodes was transferred onto the substrate by photolithography. Pt was sputtered over the surface under the same conditions as above, with the exception that sputtering was performed at room temperature so as not to hard-bake the resist. Lift-off of the resist and unwanted Pt 


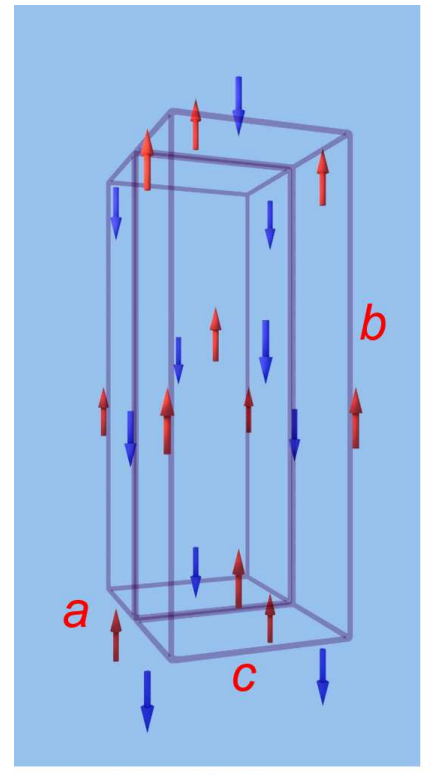

$M=\mathrm{Ni}, \mathrm{Fe}$

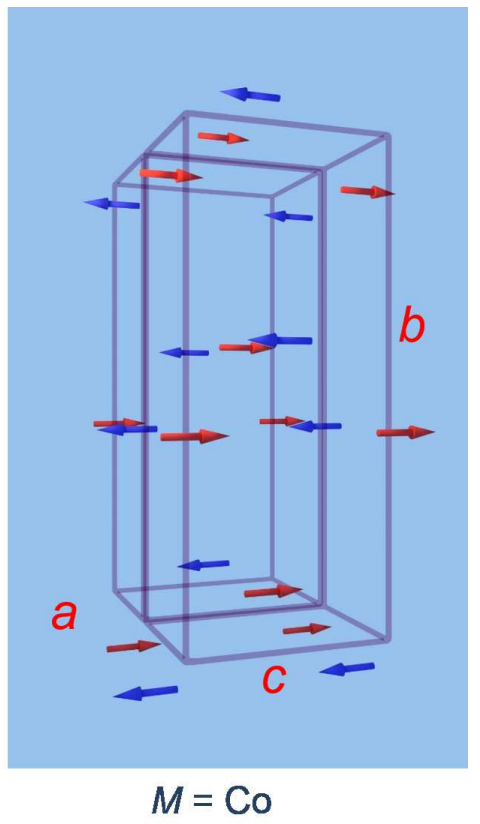

$M=\mathrm{Co}$

Figure 4.3: Diagram of the collinear $\mathrm{AF}$ spin configuration in $\mathrm{BaFeF}_{4}, \mathrm{BaNiF}_{4}$ (left), and $\mathrm{BaCoF}_{4}$ (right).

was performed by soaking the sample in acetone warmed on a hot plate. The first variation was used to apply electric fields perpendicular to the film plane while the second variation was used to apply the field in the film plane as shown in figure 4.4.

Once loaded into the MBE growth chamber, all substrates were annealed in vacuum at $700{ }^{\circ} \mathrm{C}$ for one hour before reducing the temperature to the desired growth temperature and allowing sufficient time for thermal equilibrium to be reached. Films of $\mathrm{BaMF}_{4}$ were deposited by co-deposition of $\mathrm{BaF}_{4}$ and the appropriate transition metal fluoride. Therefore, prior to the beginning of growth the deposition rates of each material had to be carefully adjusted to achieve the proper stoichiometry. In the cases of $\mathrm{BaCoF}_{4}$ and $\mathrm{BaNiF}_{4}$, all three materials were evaporated from k-cells. The $\mathrm{BaF}_{2} \mathrm{k}$-cell was set to a temperature of $1080-1090{ }^{\circ} \mathrm{C}$ and its deposition rate measured by an in-situ quartz crystal monitor (QCM) (typically around $0.045 \AA / \mathrm{s}$ ). In order to achieve a one-to-one ratio of $\mathrm{BaF}_{2}$ to $M \mathrm{~F}_{2}$ the deposition rates must be 


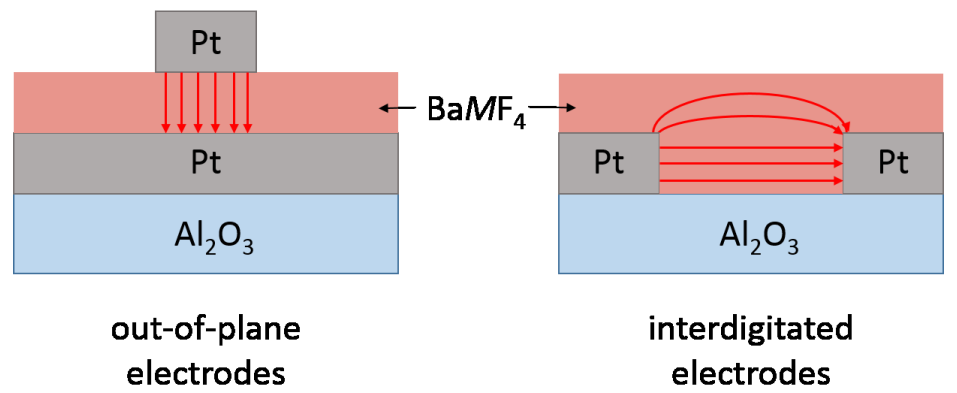

Figure 4.4: Two variations of electrodes for ferroelectric testing. Films grown on a conductive layer can be topped by metallic electrodes to apply electric fields out of plane (left). Interdigitated electrodes (shown in cross-section at right) allow for the application of in-plane electric fields.

inversely proportional to the corresponding densities according to

$$
\frac{R_{B a F_{2}}}{R_{M F_{2}}}=\frac{\rho_{M F_{2}}}{\rho_{B a F_{2}}},
$$

where $R$ is the deposition rate and $\rho$ is the atomic density. A table of the densities use for calibrating deposition rates for our samples is given in table 4.1. Therefore, the temperature of the $M \mathrm{~F}_{2}$ cell was adjusted until its measured deposition rate was in the correct proportion to the previously measured value for $\mathrm{BaF}_{2}$. In the case of $\mathrm{FeF}_{2}$, the deposition source was an e-beam evaporator. The deposition rate of that system was controlled by a computer that uses feedback from a QCM near the source to maintain a constant rate of evaporation, which can only be set in discrete units of $0.1 \AA /$ s at the source. Because finer control of the deposition rate could be obtained with k-cells, the temperature of the $\mathrm{BaF}_{2}$ cell was adjusted to match with a pre-programmed $\mathrm{FeF}_{2}$ depositon rate. 
4.3. RESULTS AND DISCUSSION

\begin{tabular}{c|cc}
\hline \hline Di-fluoride & atomic density $\left(\AA^{-3}\right)$ & $\rho_{M F_{2}} / \rho_{B a F_{2}}$ \\
\hline $\mathrm{Fe}$ & 0.0273 & 1.629 \\
$\mathrm{Co}$ & 0.0284 & 1.695 \\
$\mathrm{Ni}$ & 0.0299 & 1.784 \\
$\mathrm{Ba}$ & 0.0168 & 1.000 \\
\hline \hline
\end{tabular}

Table 4.1: Atomic densities of di-fluorides and their ratios with respect to $\mathrm{BaF}_{2}$.

\subsection{Results and Discussion}

\subsubsection{Structure}

The selection of an appropriate substrate presented a significant challenge to the successful growth of $\mathrm{BaMF}_{4}$ films because it must accommodate an orthorhombic unit cell with a low degree of lattice mismatch at the interface while orienting the ferroelectric $\mathrm{x}$-axis in a direction amenable to ferroelectric characterizatiion. Early attempts to grow $\mathrm{BaCoF}_{4}$ on $\mathrm{Si}$ (100) and (110) substrates proved unsuccessful. Following the work of Aizawa et al. [94] on the growth of (120) oriented $\mathrm{BaMgF}_{4}$ thin films on Pt (111) surfaces, we attempted to replicate those results for $\mathrm{BaCoF}_{4}$. Films grown between 200 and $400{ }^{\circ} \mathrm{C}$ showed a clear progression in crystal quality with growth temperature, as observed in the XRD data shown in figure 4.5. However, the films favored a (010) growth orientation, and consequently had their ferroelectric axis in the plane of the sample. At $500{ }^{\circ} \mathrm{C}$ the material failed to adhere properly to the substrate. The effusion temperature of the $\mathrm{CoF}_{2}$ source was only around $640{ }^{\circ} \mathrm{C}$, so in all likelihood the material desorbed from the hot substrate. Figure 4.6 shows an AFM image of the best of this series of films. Note that large single crystal grains on the order of several hundreds of nanometers in size are visible. Attempts to measure polarization vs. electric field loops poled between the epitaxial Pt underlayer and Pt surface electrodes did not yield any unambiguous evidence of ferroelectric behavior. 
The films were sufficiently rough that the contacts frequently shorted across the film. Moreover, the use of a conductive growth surface precluded any poling in the plane of the sample where ferroelectric switching would be expected to occur. An attempt was also made to measure out of plane PFM on these films, but without success. For these reasons, the Pt layer was abandoned and growth was attempted directly on the bare $\mathrm{Al}_{2} \mathrm{O}_{3}$ (001) surface. As can be seen in figure 4.12, approximately four rectangular (101) unit cells of $\mathrm{BaMF}_{4}$ can fit across five (0001) unit cells of $\mathrm{Al}_{2} \mathrm{O}_{3}$ with a tensile strain along the $a$-axis between $1.8 \%$ and $2.9 \%$, and a compressive strain along the $c$-axis between $2.0 \%$ and $2.8 \%$ across the three materials.

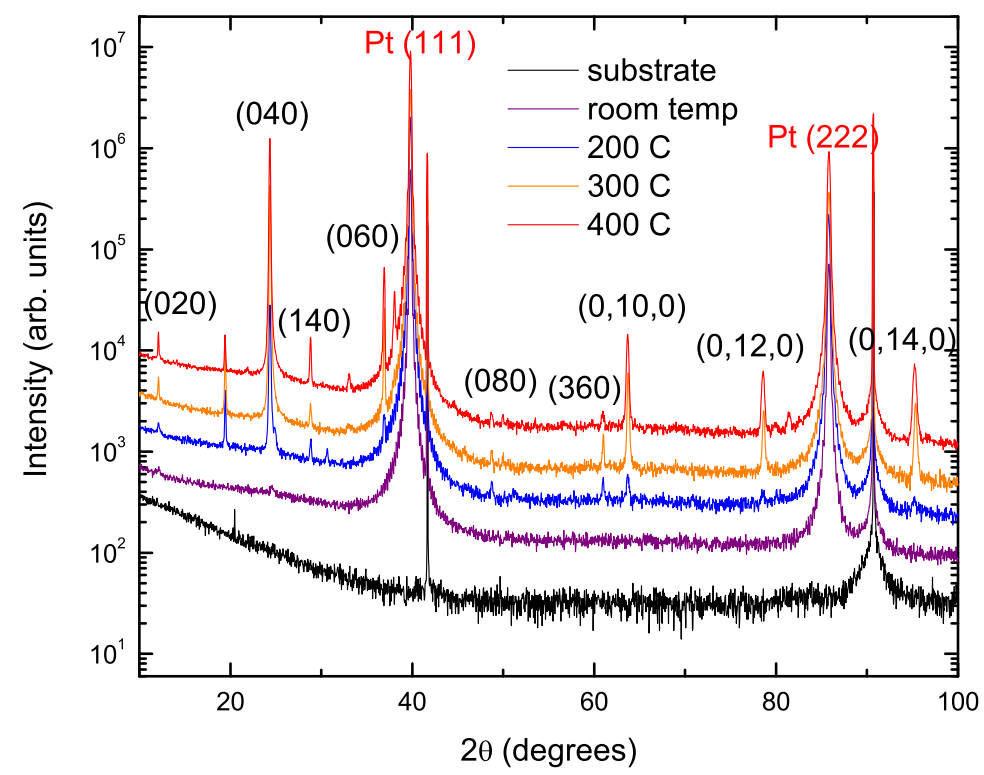

Figure 4.5: Out-of-plane XRD vs temperature of $\mathrm{BaCoF}_{4}$ samples grown on $\mathrm{Pt}(111)$.

Samples of $\mathrm{BaCoF}_{4}, \mathrm{BaFeF}_{4}$, and $\mathrm{BaNiF}_{4}$ were grown over temperature ranges of $300-500{ }^{\circ} \mathrm{C}$ to optimize the crystal quality. Out-of-plane XRD scans showed that $\mathrm{BaFeF}_{4}$ had the lowest ideal growth temperature at $\mathrm{T}=300{ }^{\circ} \mathrm{C}$, then $\mathrm{BaCoF}_{4}$ at 


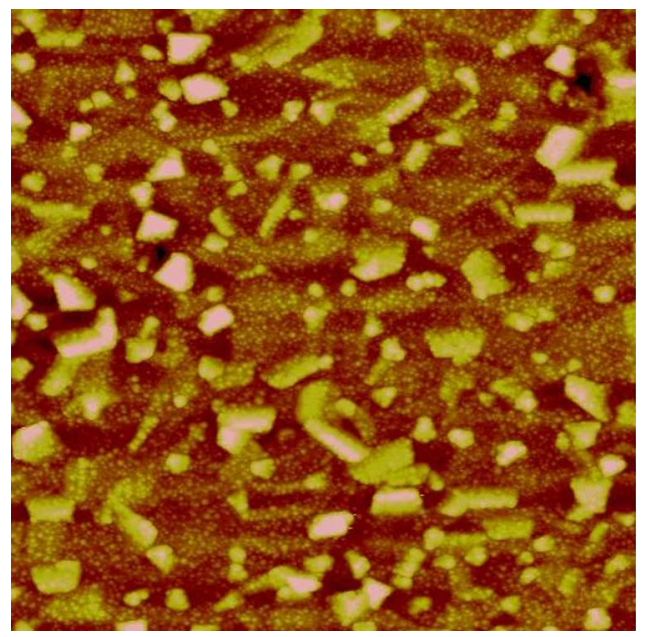

Figure 4.6: AFM image $\left(3 \times 3 \mu \mathrm{m}^{2}\right)$ of a $100 \mathrm{~nm}$ thick $\mathrm{BaCoF}_{4}$ film grown on $\mathrm{Pt}$ (111) at $400{ }^{\circ} \mathrm{C}$. Large angular crystals on the order of several hundred nanometers are visible in the surface topography. The $z$-axis color scale runs to $50 \mathrm{~nm}$.

$\mathrm{T}=350-400{ }^{\circ} \mathrm{C}$, and $\mathrm{BaNiF}_{4}$ had the highest temperature with the best sample grown at $\mathrm{T}=450{ }^{\circ} \mathrm{C}$. In most cases, the films were deposited simultaneously on two $\mathrm{Al}_{2} \mathrm{O}_{3}$ substrates placed side by side, one blank, and the other with Pt interdigitated electrodes. The total compound deposition rates varied between $0.0484-0.0824 \AA / \mathrm{s}$ for $\mathrm{BaFeF}_{4}, 0.0625-0.0741 \AA / \mathrm{s}$ for $\mathrm{BaCoF}_{4}$, and $0.0583-0.0666 \AA / \mathrm{s}$ for $\mathrm{BaNiF}_{4}$. The samples of $\mathrm{BaCoF}_{4}$ and $\mathrm{BaNiF}_{4}$ were grown to a thickness of $100 \mathrm{~nm}$, necessitating growth times of over 4 hours. Due to the greater instability of the e-beam source deposition rate for $\mathrm{FeF}_{2}$ over such a protracted period of time the $\mathrm{BaFeF}_{4}$ samples were grown to a thickness of only $50 \mathrm{~nm}$.

The quality of the samples was monitored in vacuum via RHEED immediately after deposition. The most typical pattern observed was similar to the image of a $\mathrm{BaCoF}_{4}$ sample shown in figure 4.7. A set of diffuse spots appeared to be ordered in an array. This is neither the set of streaks one would associate with a single crystal film nor the ring pattern characteristic of a completely polycrystalline sample. Rather, this pattern is suggestive of a high degree of regular crystal order, but with 
several distinct orientations present.

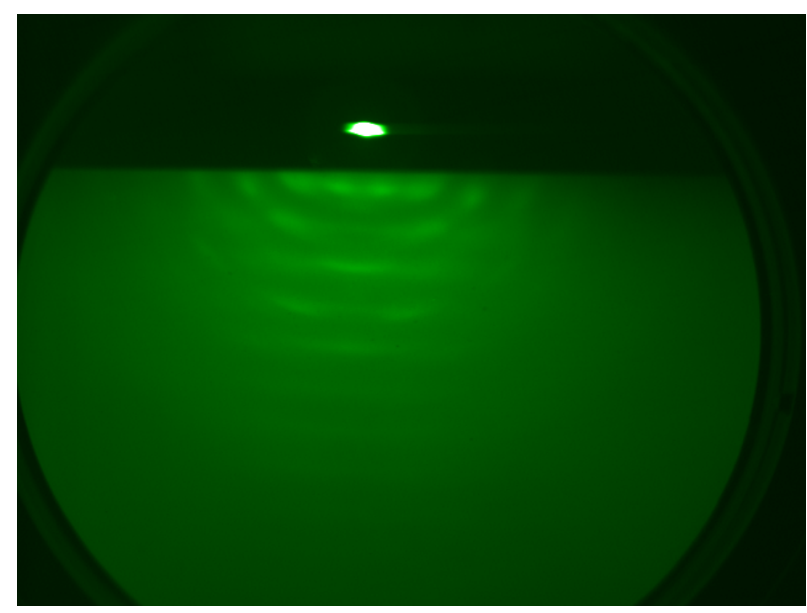

Figure 4.7: RHEED image of a high crystal quality $\mathrm{BaCoF}_{4}$ film immediately after growth.

For all three materials, the growth was preferential in the (010) orientation as seen in the x-ray diffraction data in figures 4.8 to 4.10 . In-plane $\Phi$-scans showed that all of the films had a three-fold twinning as seen in figure 4.11 for a representative sample of $\mathrm{BaCoF}_{4}$. During the in-plane scan the sample is rotated about the [010] direction, the (140) and (111) orientations should display 2 and 4 peaks, respectively, due to the orthorhombic symmetry of the crystal. However, three such sets of peaks were observed with a $120^{\circ}$ separation between them. Additionally, the separation of the (111) peaks belonging to a single orientation is indicative of the ratio between the $a$ and $c$ lattice parameters and is consistent with the structural analysis discussed below. The orientation of these domains relative to the substrate is as indicated in figure 4.12, with the polar $a$-axis of one domain parallel to the $a$-axis of the $\mathrm{Al}_{2} \mathrm{O}_{3}$.

Out-of-plane XRD on the highest quality $\mathrm{BaFeF}_{4}$ sample (figure 4.8) showed, in addition to the principle (010) ordering of the film, a collection other peaks associated both with a weak (002) phase of the $\mathrm{BaFeF}_{4}$ as well as peaks associated with $\mathrm{FeF}_{2}$ and $\mathrm{BaF}_{2}$. While the overall quality of the film was comparable to that obtained for 


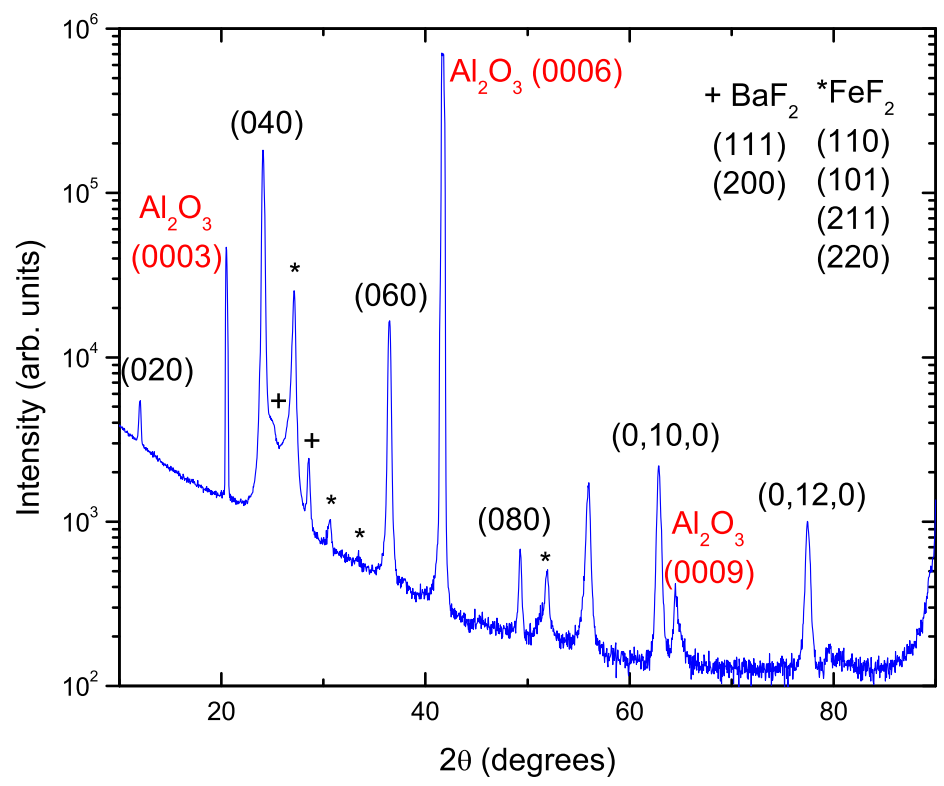

Figure 4.8: Out-of-plane XRD scan of $\mathrm{BaFeF}_{4}(50 \mathrm{~nm})$ grown at $300{ }^{\circ} \mathrm{C}$ on $\mathrm{Al}_{2} \mathrm{O}_{3}$ (0001).

$\mathrm{BaCoF}_{4}$ and $\mathrm{BaNiF}_{4}$ films, the formation of these additional phases is attributable to the relative instability of the e-beam source as opposed to K-cells over the duration of deposition. XRD of the best $\mathrm{BaCoF}_{4}$ film did not reveal any pure phases of the constituents, but there were secondary peaks associated with a tilting of the structure along the c-axis. These (071), (091), and (0101) peaks appeared adjacent to the b-axis orientations $(080),(0 \overline{10} 0)$, and $(0 \overline{12} 0)$, respectively, with corresponding tilt angles of $26^{\circ}, 21^{\circ}$, and $18^{\circ}$. In the case of $\mathrm{BaNiF}_{4}$, the XRD data of the highest quality sample, shown in figure 4.10, showed only a single out-of-plane phase with only a small peak attributed to $\mathrm{BaF}_{2}(320)$.

Measurement of the $b$-axis lattice parameter were directly obtained from the outof-plane scans of the (010) order peaks. Asymmetrical scans of the (140) and (280) peaks were then used to determine the $a$ lattice parameter, and finally scans of the 


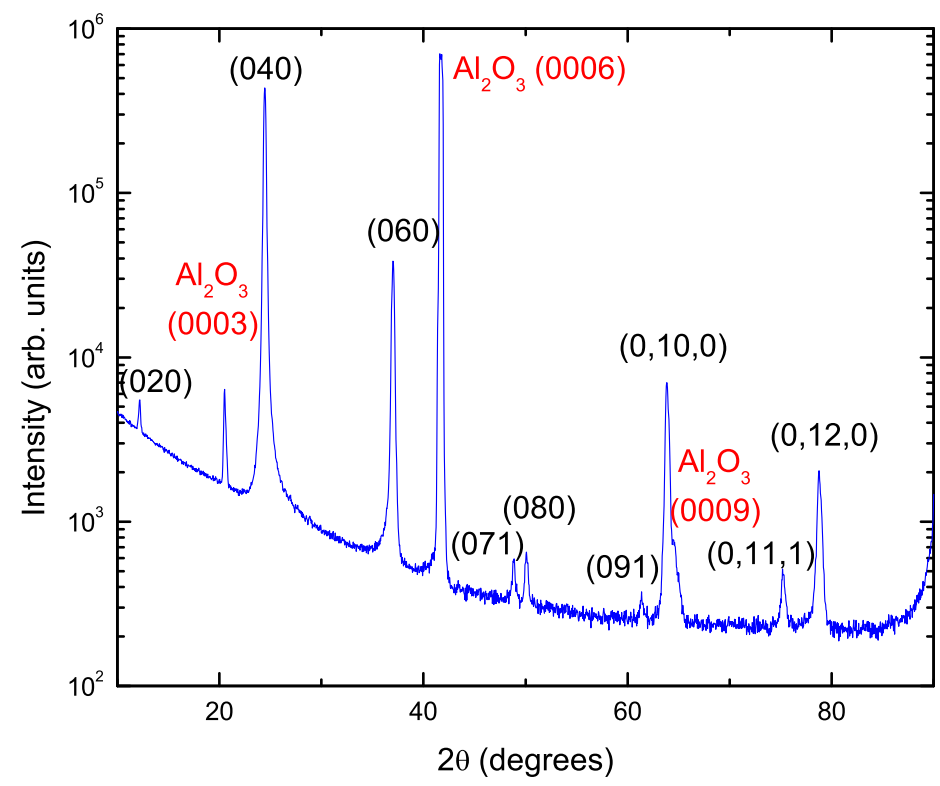

Figure 4.9: Out-of-plane XRD scan of $\mathrm{BaCoF}_{4}(100 \mathrm{~nm})$ grown at $350{ }^{\circ} \mathrm{C}$ on $\mathrm{Al}_{2} \mathrm{O}_{3}$ (0001).

(111), (222), and (333) peaks were taken to determine the $c$ lattice parameter [95]. The determined parameters of the unit cell are summarized in Table 4.2. The bulk lattice parameters obtained from the literature are presented in Table 4.3. In all three materials the $a$ lattice parameter is the most strained. For $M=\mathrm{Fe}$ and $\mathrm{Co}$ the $c$-axis lattice parameter is similar to the bulk value within the experimental uncertainties. The stretching of the in-plane footprint led to a decrease in the $b$ axis lattice parameter in these two cases. In the $M=\mathrm{Ni}$ sample, there was clear stretching of the $a$-axis lattice parameter while the change in the $b$-axis was within the experimental uncertainty of the bulk value.

The full width at half maximum (FWHM) of the rocking curves of the (040) peak for all three materials were similar for $\mathrm{BaFeF}_{4}$ and $\mathrm{BaCoF}_{4}$, but somewhat larger in $\mathrm{BaNiF}_{4}$, as shown in figure 4.13. The FWHM for each, based on a Lorentzian 


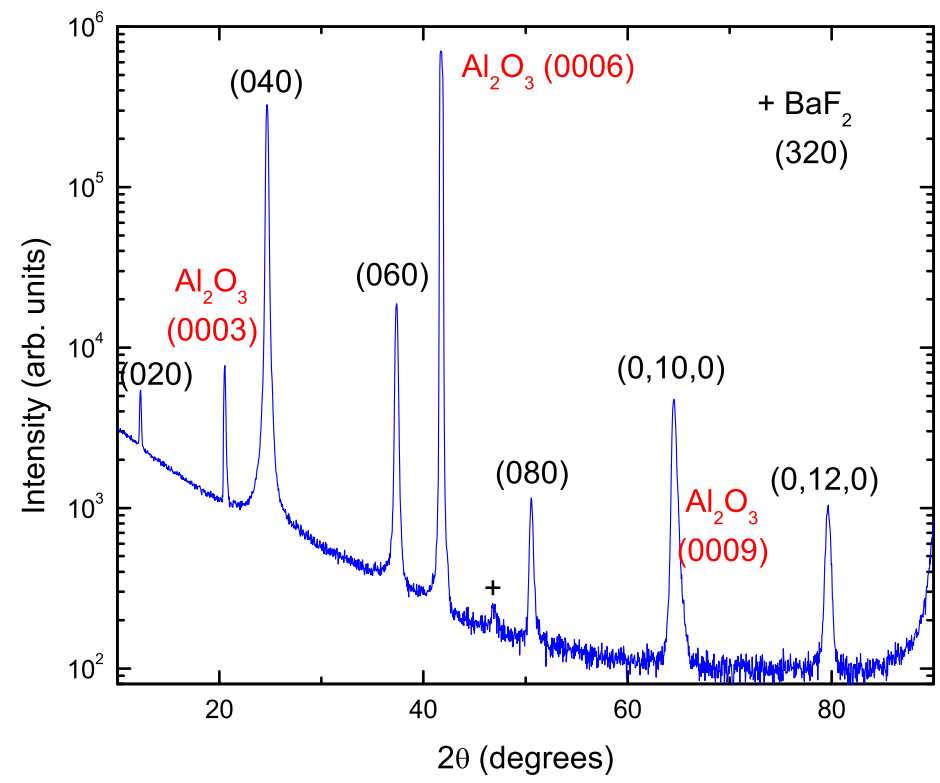

Figure 4.10: Out-of-plane XRD scan of $\mathrm{BaNiF}_{4}(100 \mathrm{~nm})$ grown at $450{ }^{\circ} \mathrm{C}$ on $\mathrm{Al}_{2} \mathrm{O}_{3}$ (0001).

fit to the data, were $0.46^{\circ}, 0.58^{\circ}$, and $1.00^{\circ}$, respectively. Taken as an indicator of the structural quality of the samples, the relatively narrow rocking curve observed in $\mathrm{BaFeF}_{4}$ suggests that the alternate phases seen in the corresponding XRD outof-plane profile are not the result of defects in the growth, but rather originate from deviations in the stoichiometry of the constituents. The relatively larger value found in $\mathrm{BaNiF}_{4}$ is consistent with the observation that its lattice parameters have the most appreciable deviation from their bulk values. While it is of a single phase, it is likely that strain in the in-plane lattice constants caused a higher concentration of defects. The rocking curve of the (0006) peak of the substrate is also shown (FWHM $=0.25^{\circ}$, which was limited by the resolution of instrument. 


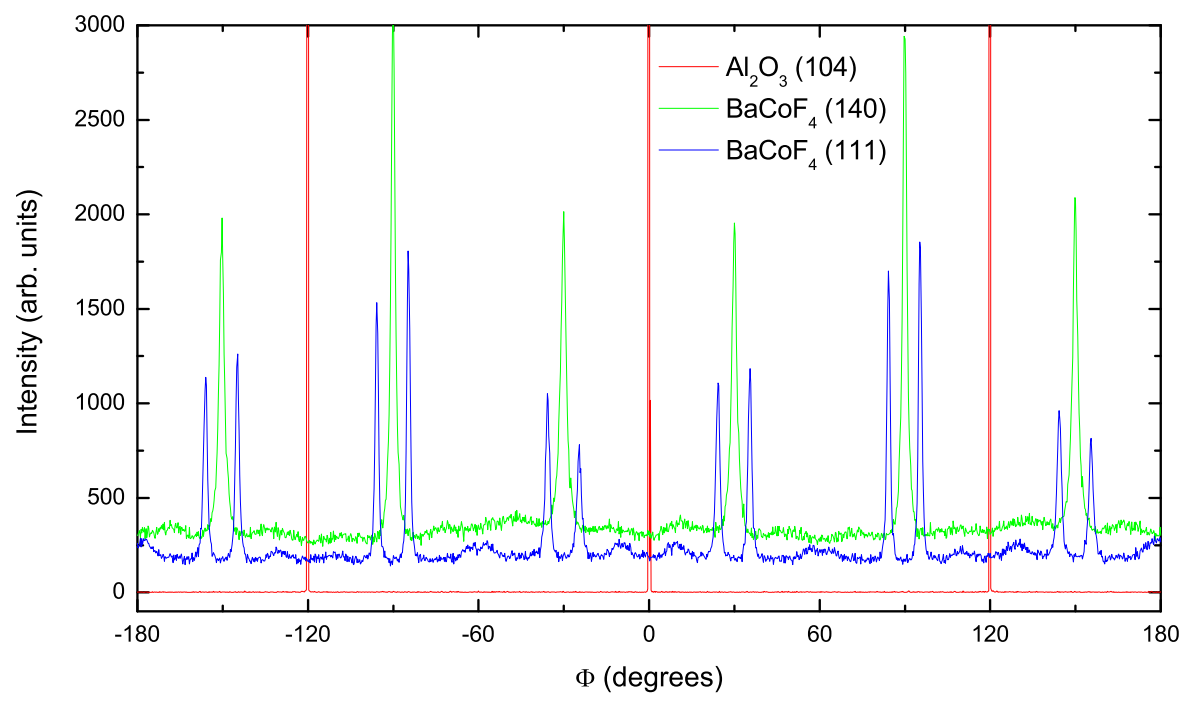

Figure 4.11: In-plane $\Phi$-scan of a $\mathrm{BaCoF}_{4}$ thin film grown on $\mathrm{Al}_{2} \mathrm{O}_{3}$ (0001) showing the three-fold twinning of the film structure.

\begin{tabular}{c|ccc}
\hline \hline$M$ & $a(\AA)$ & $b(\AA)$ & $c(\AA)$ \\
\hline $\mathrm{Fe}$ & $5.94 \pm 0.02(+1.9 \%)$ & $14.77 \pm 0.02(-0.6 \%)$ & $4.23 \pm 0.01(-0.3 \%)$ \\
$\mathrm{Co}$ & $5.93 \pm 0.01(+1.4 \%)$ & $14.56 \pm 0.01(-0.4 \%)$ & $4.20 \pm 0.01(-0.1 \%)$ \\
$\mathrm{Ni}$ & $5.88 \pm 0.01(+1.6 \%)$ & $14.44 \pm 0.01(+0.1 \%)$ & $4.18 \pm 0.02(+0.9 \%)$ \\
\hline \hline
\end{tabular}

Table 4.2: Lattice parameters for highest quality thin film samples of $\mathrm{Ba} \mathrm{F}_{4}$ obtained from XRD. The values in parentheses are the percentage deviations of the measured value of each lattice parameter from the bulk value listed in Table 4.3.

\subsubsection{Magnetic Characterization}

The magnetic moment of the samples was measured as a function of temperature via SQUID magnetometry under a procedure of first cooling in zero applied magnetic field down to $5 \mathrm{~K}$, and then measuring in a field of $1 \mathrm{kOe}$ as the temperature was swept to $320 \mathrm{~K}$. This procedure is called zero-field cooling (ZFC). With the field still applied, the moment was again measured as the temperature was swept back down to $5 \mathrm{~K}$ in a procedure called field cooling (FC). Subsequently, the field was removed 


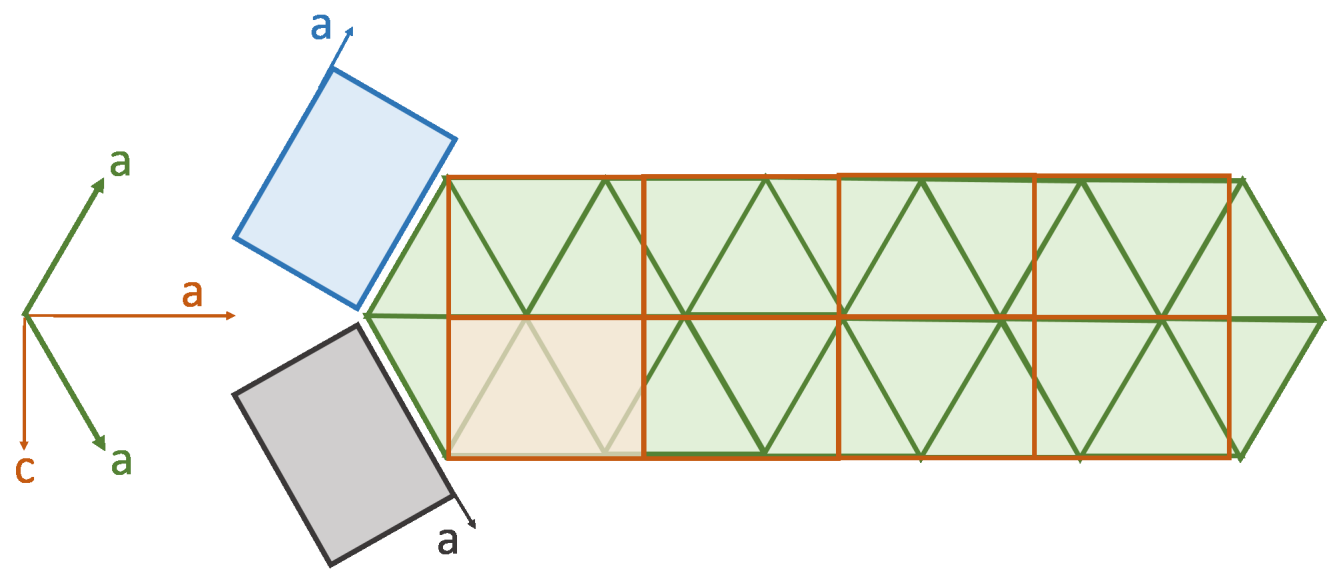

Figure 4.12: Diagram of the orientation and lattice match of three twins of $\mathrm{Ba}_{4} \mathrm{~F}_{4}$ with respect to the $\mathrm{Al}_{2} \mathrm{O}_{3}$ (0001) surface. Four unit cells of $\mathrm{Ba} M \mathrm{~F}_{4}$ can fit across five unit cells of $\mathrm{Al}_{2} \mathrm{O}_{3}$, with the mismatch giving rise to tensile strain in the $a$-axis and compressive strain along the $c$-axis.

\begin{tabular}{c|ccc}
\hline \hline$M$ & $a(\AA)$ & $b(\AA)$ & $c(\AA)$ \\
\hline $\mathrm{Fe}$ & 5.83 & 14.86 & 4.24 \\
$\mathrm{Co}$ & 5.85 & 14.63 & 4.21 \\
$\mathrm{Ni}$ & 5.78 & 14.43 & 4.14 \\
\hline \hline
\end{tabular}

Table 4.3: Lattice parameters for bulk samples of $\mathrm{Ba} \mathrm{F}_{4}$ taken from [90].

and the thermal remanent magnetization (TRM) was measured from $5 \mathrm{~K}$ to $320 \mathrm{~K}$. The measurements were performed with the magnetic field oriented parallel to the [210] direction of the substrate and in the plane, i.e. perpendicular to one $\mathrm{Ba} \mathrm{F}_{4}$ domain and at a $30^{\circ}$ angle to the other two. The diamagnetic response of a blank $\mathrm{Al}_{2} \mathrm{O}_{3}$ substrate was measured and subtracted from each measurement.

$\mathrm{BaFeF}_{4}$ exhibited behavior characteristic of a bulk antiferromagnet with a single broad peak in the ZFC curve in the range of 50 - $80 \mathrm{~K}$ (figure 4.14), consistent with the bulk antiferromagnetic transition at $70 \mathrm{~K}$. The sharp upturn in magnetization below $10 \mathrm{~K}$ is an artifact of the substrate that could not be readily subtracted, and is common to all samples. The splitting of the FC and ZFC curves below $50 \mathrm{~K}$ is 


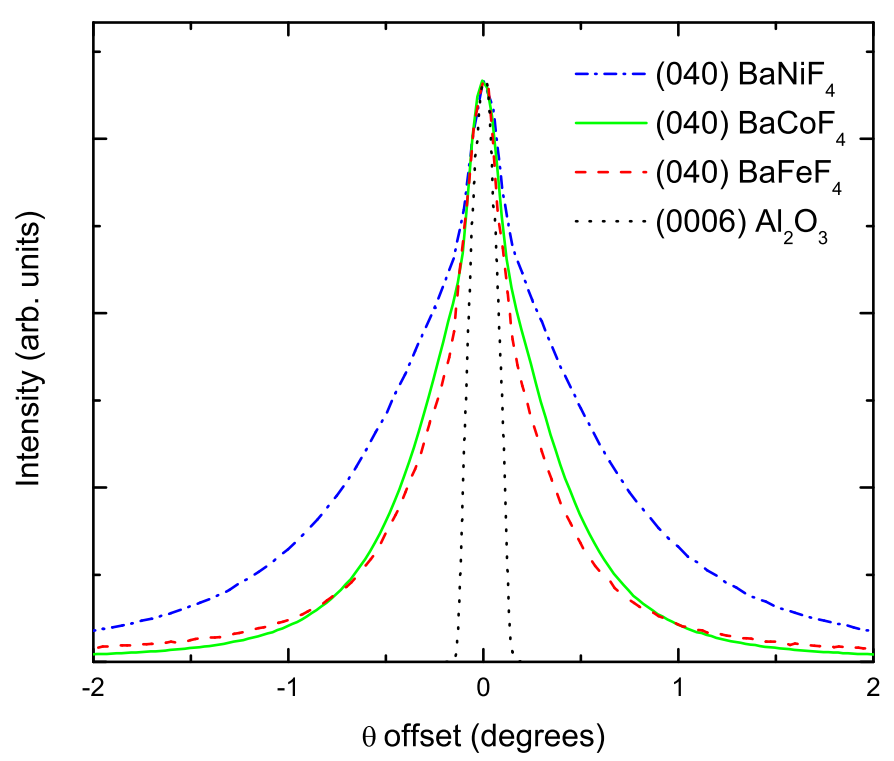

Figure 4.13: Rocking curves of the (040) peak of the $\mathrm{Ba} \mathrm{F}_{4}$ films. The rocking curve of the (0006) curve of the substrate is shown as an indicator of the instrument resolution.

indicative of a weak ferromagnetic behavior.

Magnetic hysteresis loops, shown in figure 4.15, are closed with a non-linear Sshape in low fields and at temperatures up to $200 \mathrm{~K}$. A possible explanation for this is strain-induced weak ferromagnetism [96]. The $\mathrm{Ba} \mathrm{F}_{4}$ compounds have stronger exchange interactions within the puckered $a c$-planes than between planes. Because of this, intra-plane spin ordering can persist up to $3 T_{N}$ [92]. Distortion of the $a$ and $c$ lattice parameters could distort this persistent spin ordering to account for weak ferromagnetism well above $T_{N}$. If this is the case, it could account for similar behavior observed in $\mathrm{BaCoF}_{4}$ (figure 4.18) and $\mathrm{BaNiF}_{4}$ (figure 4.20).

The magnetization vs. temperature curve of $\mathrm{BaCoF}_{4}$ also featured a broad peak in both FC and ZFC curves that is consistent with the bulk antiferromagnetic transition in the vicinity of $70 \mathrm{~K}$. The in-plane twinning of the films is uniquely relevant in 


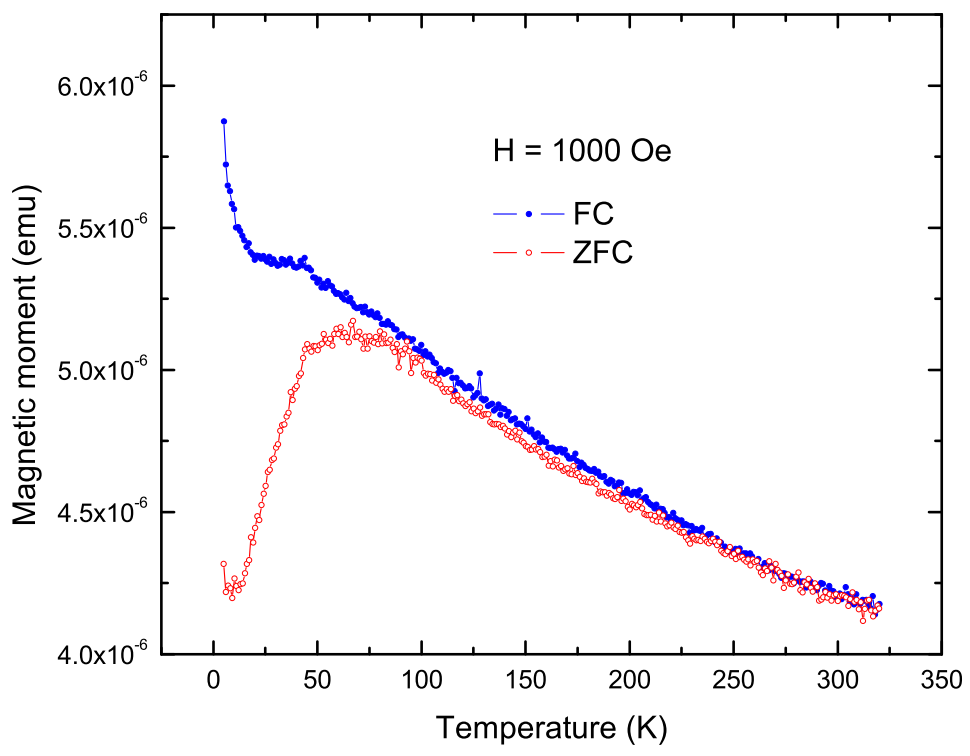

Figure 4.14: $\mathrm{FC}$ and ZFC curves for $\mathrm{BaFeF}_{4}$ showing a broad peak associated with the AF transition temperature.

$\mathrm{BaCoF}_{4}$ because it alone had its magnetic easy axis in the plane of the applied field. For one domain, the easy a-axis was perpendicular to the field while the other two were oriented at $30^{\circ}$ relative to it. Assuming that the three domains were equivalent by volume, this implies that the measured response should be a mixture of the single crystal response such that the magnetic moment should follow

$$
m=\frac{1}{2} m_{\perp}+\frac{1}{2} m_{\|}
$$

where $\chi_{\perp}$ and $\chi_{\|}$are the susceptibilities of a pure phase sample measured perpendicular and parallel to the magnetic field, respectively.

Below the main antiferromagnetic transition, $\mathrm{BaCoF}_{4}$ exhibits two additional sharp peaks, as shown in figure 4.16, that occur independently at temperatures of 


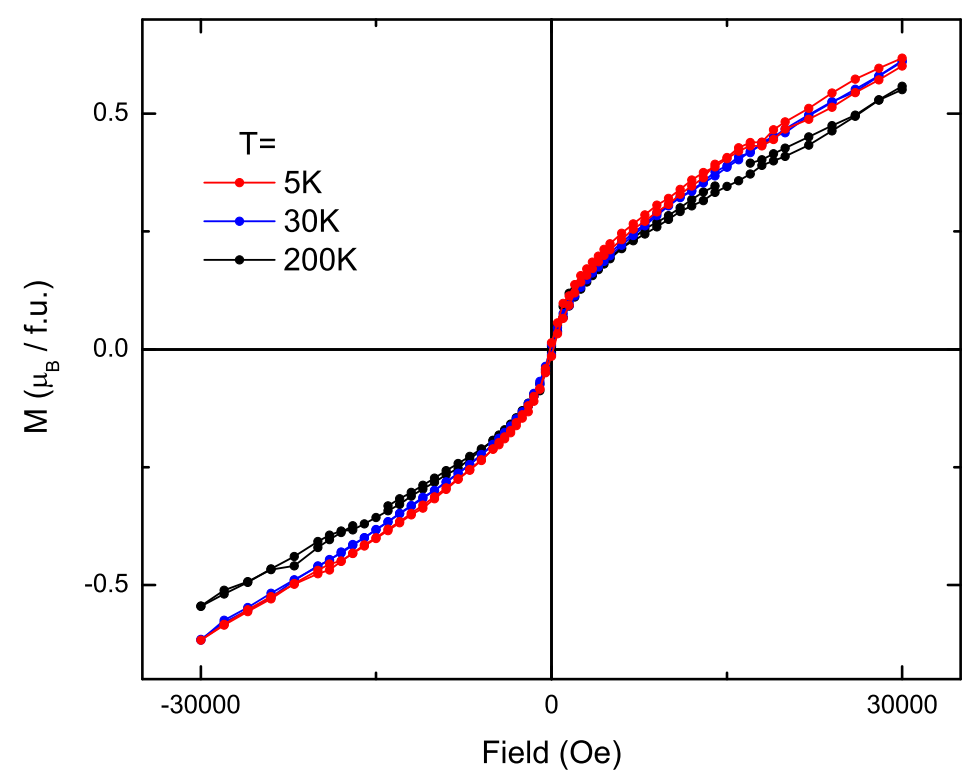

Figure 4.15: Magnetization vs. magnetic field for $\mathrm{BaFeF}_{4}$ in $\mu_{B}$ per formula unit, showing closed loops and mostly linear high field response.

$T_{c 1}=17 \mathrm{~K}$ and $T_{c 2}=41 \mathrm{~K}$. The TRM data on these two transitions (figure 4.17) shows two plateaus in the remanence below these transitions, clearly indicating that they are ferromagnetic in nature. Magnetic hysteresis loops in the temperature regions below, between, and above these two transitions are shown in figure 4.18. The loop at $5 \mathrm{~K}$ exhibits a remanence of about $0.02 \mu_{B}$ per formula unit or 112 $\mathrm{emu} / \mathrm{mol}$. This value is consistent with other weak ferromagnets, such as $\mathrm{NiF}_{2}$ [97]. Magnetic fields up to $30 \mathrm{kOe}$ were required to saturate the loop, a characteristic that can be explained by the mixture of domains in which the canting direction of the spins may be parallel (perpendicular) to the applied field, requiring low (high) fields for saturation.

A full understanding of the origin of the low temperature peaks observed in $\mathrm{BaCoF}_{4}$ has not been attained as of this writing. Preliminary work undertaken 


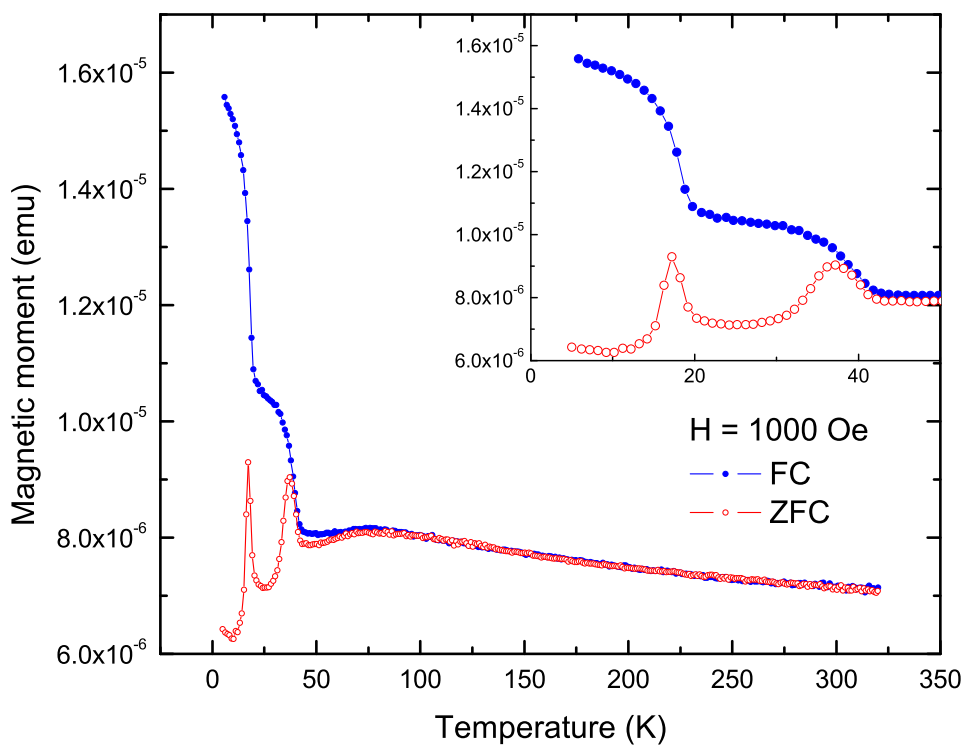

Figure 4.16: FC and ZFC curves for $\mathrm{BaCoF}_{4}$ showing a broad peak associated with the $\mathrm{AF}$ transition temperature and two low temperature magnetic transitions.

by our collaborators A. C. Garcia-Castro and Aldo Romero (WVU) based upon ab initio calculations within the framework of density functional theory, suggested noncollinear magnetic phases induced by epitaxial strain. Assuming a $B$-phase magnetic structure as the principle AF $\left(\mathbf{G}_{\mathbf{a}}\right)$ order, they determined that two subordinate order spin cantings could be allowed under strain in the $a c$-plane: a weak FM order along the $c$-axis $\left(\mathbf{F}_{\mathbf{c}}\right)$ caused by distortion of the linear Co-F-Co chains, and a weak AF $\left(\mathbf{C}_{\mathbf{b}}\right)$ order along the $b$-axis. Calculated ground state energies for these phases are shown in table 4.4 for different values of strain. Accordingly, as the temperature is increased the weak FM order with the lowest energy would vanish first, then the weak AF order, and finally the collinear AF order.

The magnetic behavior of $\mathrm{BaNiF}_{4}$ (shown in figure 4.19), like the other two materials, exhibited a broad peak in the ZFC curve in the range of $50-90 \mathrm{~K}$. 


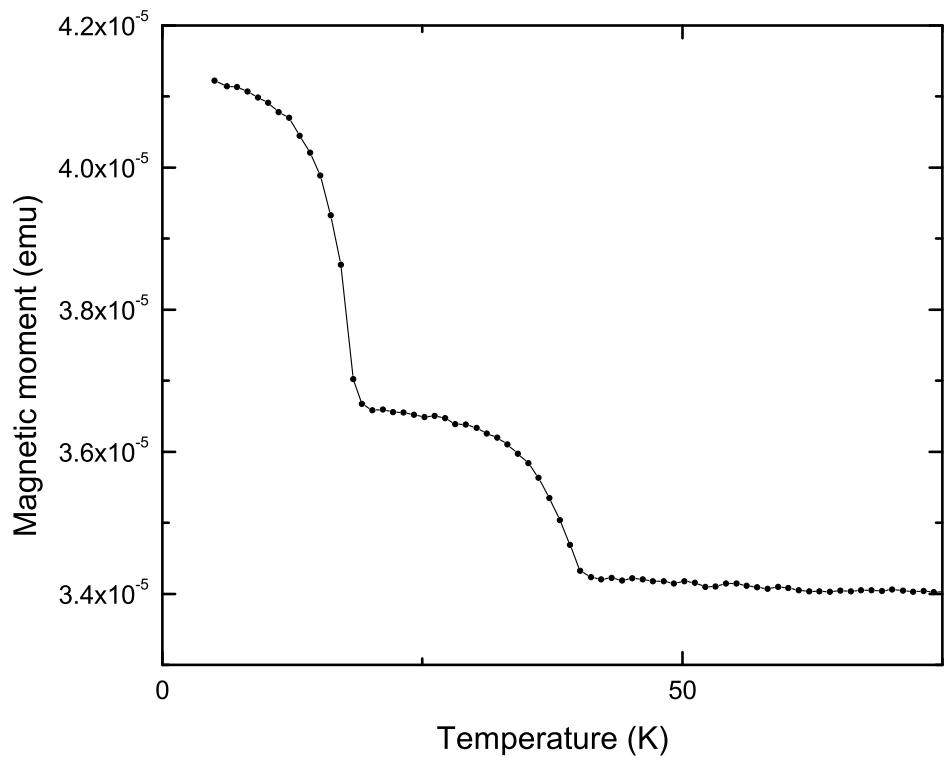

Figure 4.17: TRM of $\mathrm{BaCoF}_{4}$ showing a remanent magnetic moment that drops at the two low temperature transitions.

However, it is not as prominent as the other two, and is more difficult to distinguish due to the background noise. In addition, there is a small, but discernable peak of unknown origin at approximately $28 \mathrm{~K}$, which also appears for other samples. A little understood feature of the $\mathrm{BaNiF}_{4}$ magnetization data is the gap between the $\mathrm{ZFC}$ and FC curves that opens near room temperature. This gap appeared in three of four samples measured, and the one in which the gap did not appear, was essentially polycrystalline. Unfortunately the most optimal quality sample was lost before it could be measured, so it couldn't shed additional light on this anomaly. A possible explanation is that this high temperature magnetic behavior could have resulted from impurities in the $\mathrm{NiF}_{2}$ source material. To check this, a film of pure $\mathrm{NiF}_{2}$ was accordingly grown and magnetically characterized with the result that there was no high temperature anomaly. Therefore, this behavior appears to be intrinsic to the 


\begin{tabular}{cccc}
\hline \hline \multirow{2}{*}{ Strain } & \multicolumn{3}{c}{$\mathrm{E}_{t}(\mathrm{eV} /$ atom $)$} \\
\cline { 2 - 4 } & $\mathrm{E}\left(G_{a} C_{b}\right)$ & $\mathrm{E}\left(G_{a} C_{b} F_{c}\right)$ & $\Delta \mathrm{E}\left(G_{a} C_{b}-G_{a} C_{b} F_{c}\right)$ \\
\hline$-4 \%$ & -5.5746 & -5.5746 & $-0.006\left(\times 10^{-3}\right)$ \\
$0 \%$ & -5.5988 & -5.5988 & $+0.010\left(\times 10^{-3}\right)$ \\
$+1.2 \%$ (exp.) & -5.5786 & -5.5978 & $+19.205\left(\times 10^{-3}\right)$ \\
$+4 \%$ & -5.5627 & -5.55627 & $-0.079\left(\times 10^{-3}\right)$ \\
\hline \multicolumn{3}{c}{$G_{a} C_{b} F_{a} m_{i}\left(\mu_{B} /\right.$ Co-atom $)$} \\
\hline \multirow{2}{*}{ Strain } & \multicolumn{3}{c}{$C_{b}$} \\
\cline { 2 - 4 } & $G_{a}$ & $0.101,0.078$ & $F_{c}$ \\
\hline $1.2 \%$ & 2.766 & 0.034 \\
\hline \hline
\end{tabular}

Table 4.4: Calculated energies of the magnetic transitions in $\mathrm{BaCoF}_{4}$ under different conditions of lattice strain (top). Calculated magnetization of $\mathrm{BaCoF}_{4}$ under experimentally determined strain [98].

$\mathrm{BaNiF}_{4}$ films. It is perhaps due to a phase induced by the relatively higher strain in $\mathrm{BaNiF}_{4}$, but further work needs to be done to resolve this issue.

\subsubsection{Ferroelectric Characterization}

Measurements of the polarization vs. electric field were measured on the films deposited on top of Pt interdigitated electrodes via a Precision LC tester from Radiant Technologies. The electrodes used for testing consisted of 25 pairs of digits, each $0.5 \mathrm{~mm}$ in length and $50 \mathrm{~nm}$ thick, with a $2.5 \mu \mathrm{m}$ spacing between each digit and its neighbors. A picture of the electrodes with the poling direction labeled is shown in figure 4.21. Treating the set of digits as a set of parallel plate capacitors configured in parallel, this yields a net plate area of $1.225 \times 10^{-5} \mathrm{~cm}^{2}$. The electrodes were aligned such that the poling direction, i.e. the direction orthogonal to the digits in the plane, was perpendicular to the ferroelectric a-axis for one of the twins and at $30^{\circ}$ to the other two domains. Therefore, neglecting fringing effects, and assuming the three in-plane crystal orientations are equally weighted, the measured response should be $1 / \sqrt{3}$ or approximately $58 \%$ of the single domain 


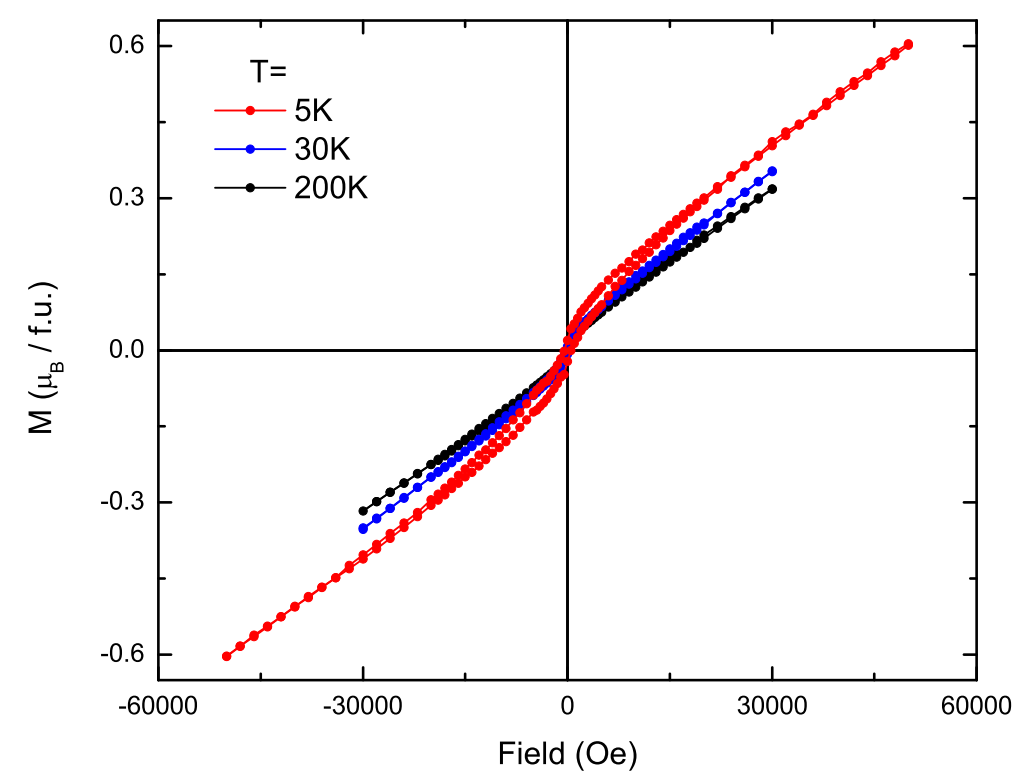

Figure 4.18: Magnetization vs. magnetic field for $\mathrm{BaCoF}_{4}$ in $\mu_{B}$ per formula unit, showing an open loop at $5 \mathrm{~K}$.

polarization. The leakage of the samples at room temperature was generally sufficiently high that reliable hysteresis loops could not be taken, as the current to the test system tended to exceed the current limitations of the tester $(0.1 \mathrm{~mA})$ under the high voltages necessary to induce switching. To minimize the leakage, the samples were mounted in a cryostat and cooled to a base temperature of about $14 \mathrm{~K}$. Wires running through the cryostat to the cold finger were connected to the interdigitated electrodes with indium on the one end and to the external tester on the other. The drawback of this approach was that the wires running through the cryostat had no electrical shielding and hence contributed a large linear capacitance to the measured hysteresis loop, which in addition to the intrinsic capacitance of the electrodes and leakage effects, obscured the component of the signal due to ferroelectric switching. To remove these contributions, remanent hysteresis loops were measured, as outlined 


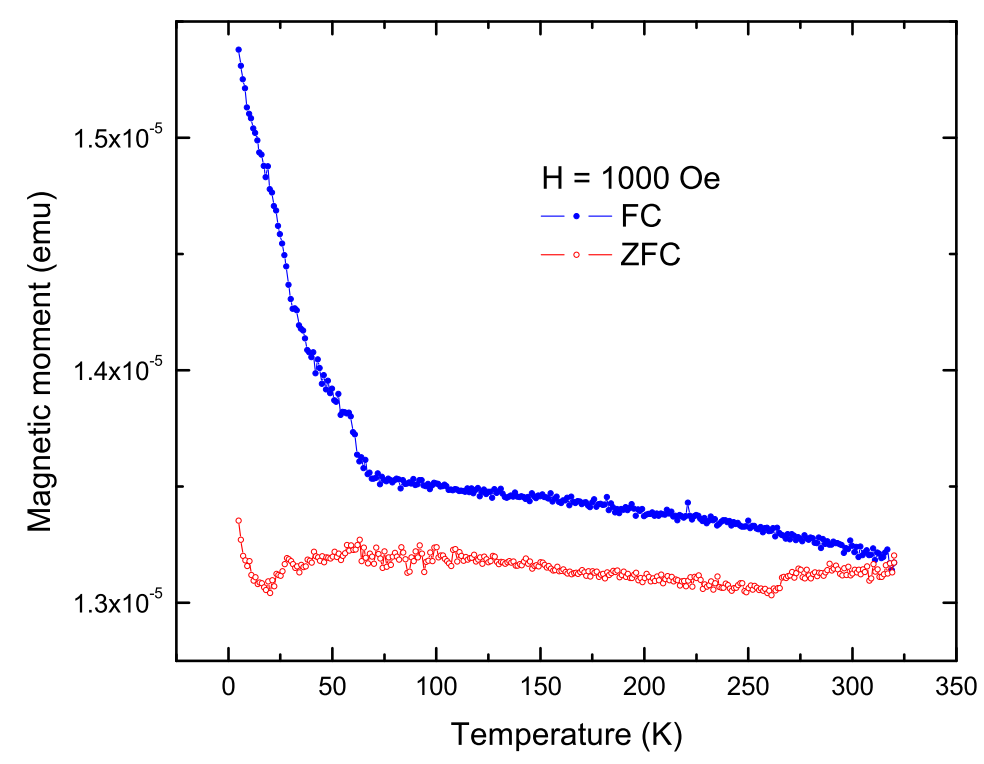

Figure 4.19: FC and ZFC curves for $\mathrm{BaNiF}_{4}$ showing a broad peak in the ZFC curve associated with the AF transition temperature and an anomalous high temperature behavior.

in chapter 2, and averaged over a large number of iterations to reduce noise. To test the reliability of this procedure, we measured remanent hysteresis of a definitively non-ferroelectric sample $\left(\mathrm{BaF}_{2}\right.$ on $\left.\mathrm{Al}_{2} \mathrm{O}_{3}\right)$ using identical interdigitated electrodes, and found no response.

Remanent hysteresis loops (see chapter 2, section 3.8) taken on $\mathrm{BaCoF}_{4}$ at a range of electric fields ranging from $108-396 \mathrm{kV} / \mathrm{cm}$ are shown in figure 4.22. Each loop represents an average of 250 remanent loops. The remanent polarization of the largest loop is approximately $2.3 \mu \mathrm{C} / \mathrm{cm}^{2}$, which is of the same order of magnitude as the $8.0 \mu \mathrm{C} / \mathrm{cm}^{2}$ measured by Eibschütz et al. in bulk [84]. There are several things to note about this value. For one, the polarization never seems to completely saturate even at fields approaching $400 \mathrm{kV} / \mathrm{cm}$. However, the component of the electric field along the ferroelectric axis of the two measurable domains was actually 


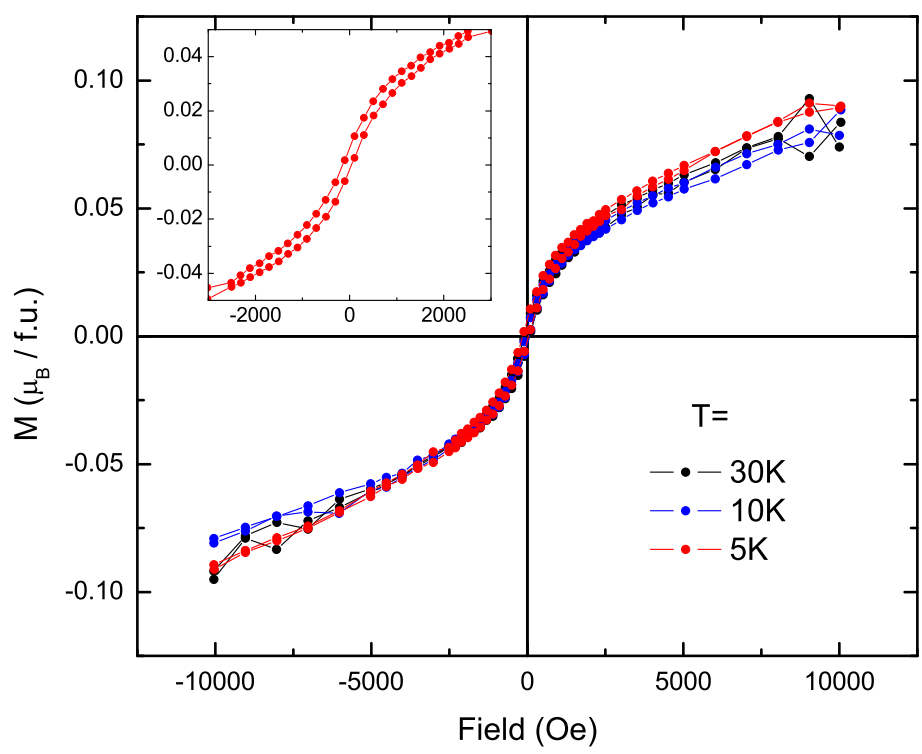

Figure 4.20: Magnetization vs. magnetic field for $\mathrm{BaNiF}_{4}$ in $\mu_{B}$ per formula unit, showing weak FM behavior at low temperature. Inset shows a magnification of the hysteresis loop at $5 \mathrm{~K}$.

smaller by a factor of $\sqrt{3} / 2$. The maximum applicable field was determined by two factors: the maximum voltage output of the tester $(99 \mathrm{~V})$, and the spacing of the electrodes (effectively limited by the resolution of the UV photolithography process to $\sim 1 \mu \mathrm{m}$ ). If either of these limitations could have been overcome, some higher value of the saturation presumably would be obtained. The orientation of the three domains would tend to underestimate the polarization in the aforementioned manner by a factor of $\sqrt{3}$. On the other hand, the effective area of the capacitor used by the tester to convert charge to polarization was underestimated because the fringing field of the electrodes would have partially polarized the film. This would have the corresponding effect of overestimating the polarization. Taking all these things into consideration, it must be concluded that the thin film polarization is not precisely known. Our results only confirm that the saturation polarization must be of the 


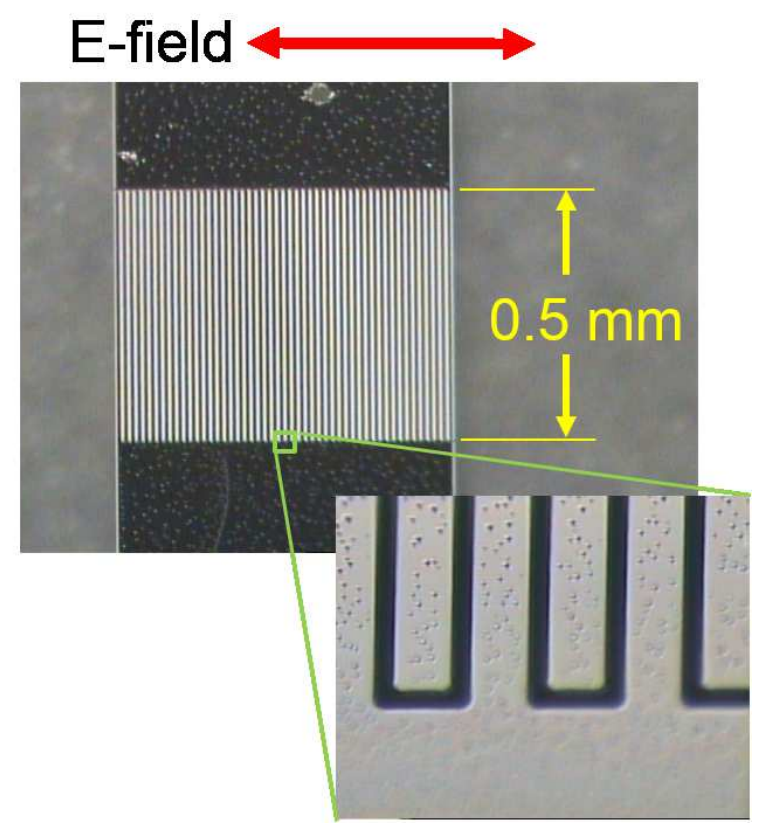

Figure 4.21: Microscope image of interdigitated Pt electrodes with $2.5 \mu \mathrm{m}$ spacing used for ferroelectric testing.

same order of magnitude as previous bulk measurements.

Among other things of note regarding these data is that it was not taken from among the higher quality samples in terms of crystal structure. XRD analysis revealed only a rather weak trace of the (040) and (060) peaks. It was mentioned above that in most cases a blank sample and one with electrodes were grown simultaneously, but this was not always the case. The best sample of $\mathrm{BaCoF}_{4}$ was produced without such a partner. Testing of another sample with somewhat better crystal quality also showed ferroelectric hysteresis, albeit with a lower remanence of $1.1 \mu \mathrm{C} / \mathrm{cm}^{2}$. Those data are shown in figure 4.23. Also, note that there is a gap of approximately $0.15 \mu \mathrm{C} / \mathrm{cm}^{2}$ between the positive and negative field regions of the loop. As mentioned in chapter 2, the remanent measurement takes the forward and backward biased half-loops as separate measurements. The tester software then affixes them together to form a single loop. While we do not have an explanation 


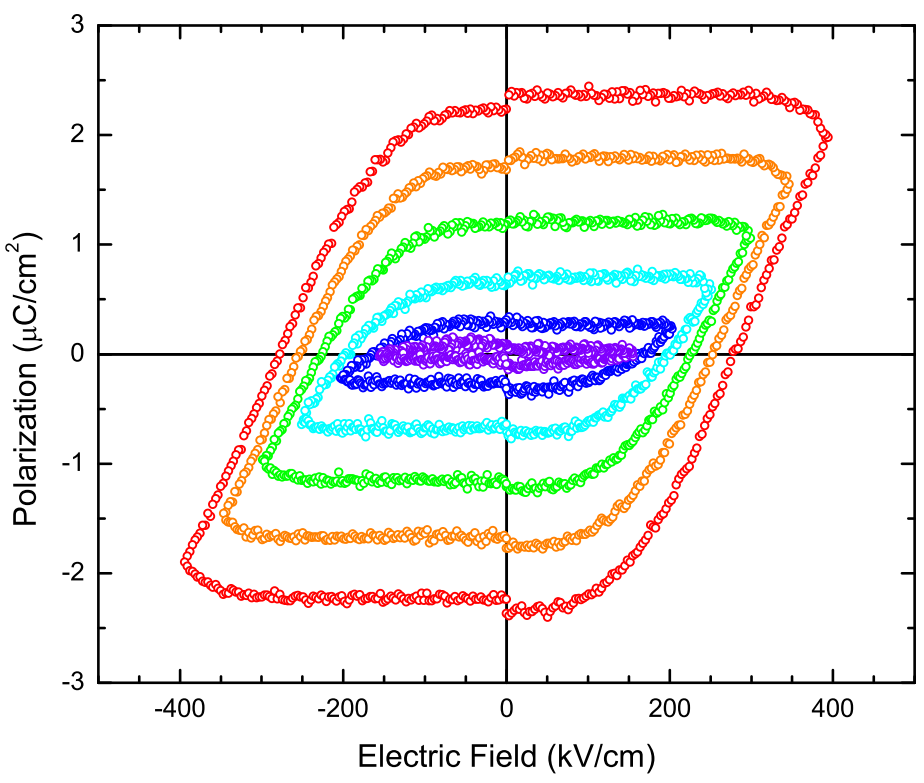

Figure 4.22: Averaged remanent polarization vs. electric field for $\mathrm{BaCoF}_{4}$ film with remanent polarization of $2.3 \mu \mathrm{C} / \mathrm{cm}^{2}$.

for this discrepancy, it is most likely an artifact of the remanent hysteresis testing procedure together with the rounding of the corners of the loop seen in the figure. Whatever its origin, the size of the gap is probably a good indication of the error in the measured value of the remanent polarization, approximately $6 \%$.

Figure 4.24 shows the averaged remanent hysteresis loops for $\mathrm{BaNiF}_{4}$. All the experimental conditions for measurement were identical to those employed for the above sample of $\mathrm{BaCoF}_{4}$. The remanence, in this case, was about $0.9 \mu \mathrm{C} / \mathrm{cm}^{2}$, which is again less than, but in the same order of magnitude as the $6.7 \mu \mathrm{C} / \mathrm{cm}^{2}$ measured in bulk. Unlike the $\mathrm{BaCoF}_{4}$ sample however, these data were measured using the optimal quality $\mathrm{BaNiF}_{4}$ film grown simultaneously with the one used to obtain the $\mathrm{XRD}$ data of figure 4.10. In this case as well, the polarization did not completely saturate at the limiting field, suggesting that higher actual values could be obtained. 


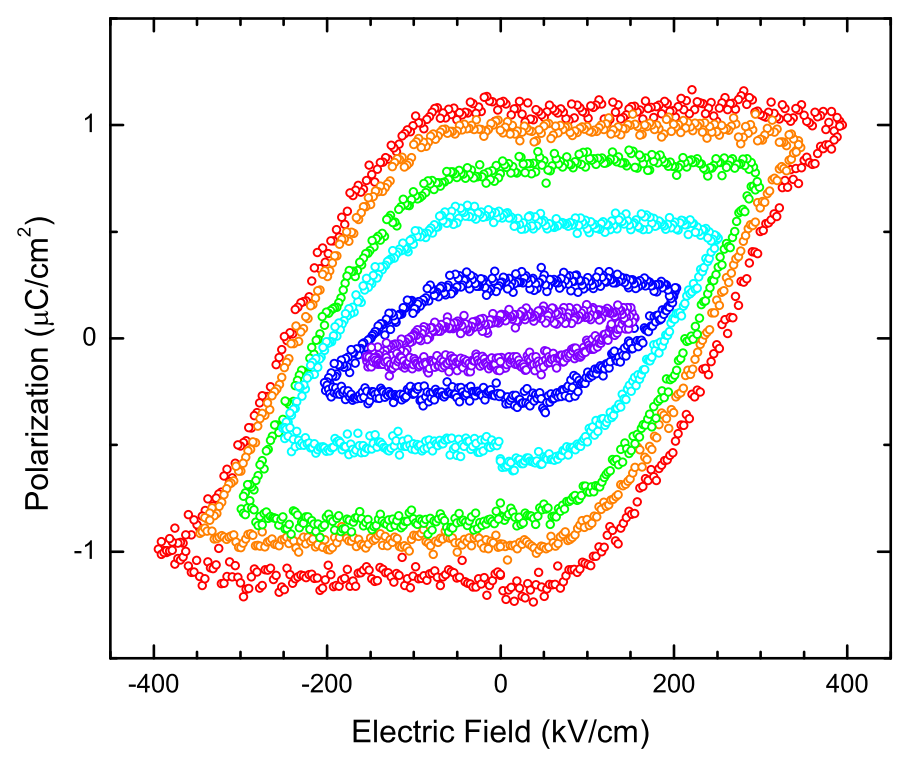

Figure 4.23: Averaged remanent polarization vs. electric field for $\mathrm{BaCoF}_{4}$ film with remanent polarization of $1.1 \mu \mathrm{C} / \mathrm{cm}^{2}$.

All attempts to measure ferroelectric hysteresis in $\mathrm{BaFeF}_{4}$ films resulted in a null result as seen in figure 4.25. Because of the difficulties associated with growing high quality $\mathrm{BaFeF}_{4}$ films, none of the samples tested were of the best structural quality. As we have seen however, this was not particularly important for $\mathrm{BaCoF}_{4}$. $\mathrm{BaFeF}_{4}$ has been predicted [90] to have a remanence on the order of $10 \mu \mathrm{C} / \mathrm{cm}^{2}$, so even samples multiple phases would be expected to have some response. Another possibility is that the limiting fields employed here $(\approx 400 \mathrm{kV} / \mathrm{cm})$ were too small to induce reversal. It could also be the case that $\mathrm{BaFeF}_{4}$ is polar, but unswitchable, i.e. it is pyroelectric. What can be concluded firmly is that the failure to obtain hysteresis was not due to extreme leakage in the samples as was claimed by Eibschütz et al. for their bulk crystal. While leakage was an evident problem in all of our $\mathrm{Ba} \mathrm{F}_{4}$ films, especially at room temperature, the leakage was actually lower in our $\mathrm{BaFeF}_{4}$ 


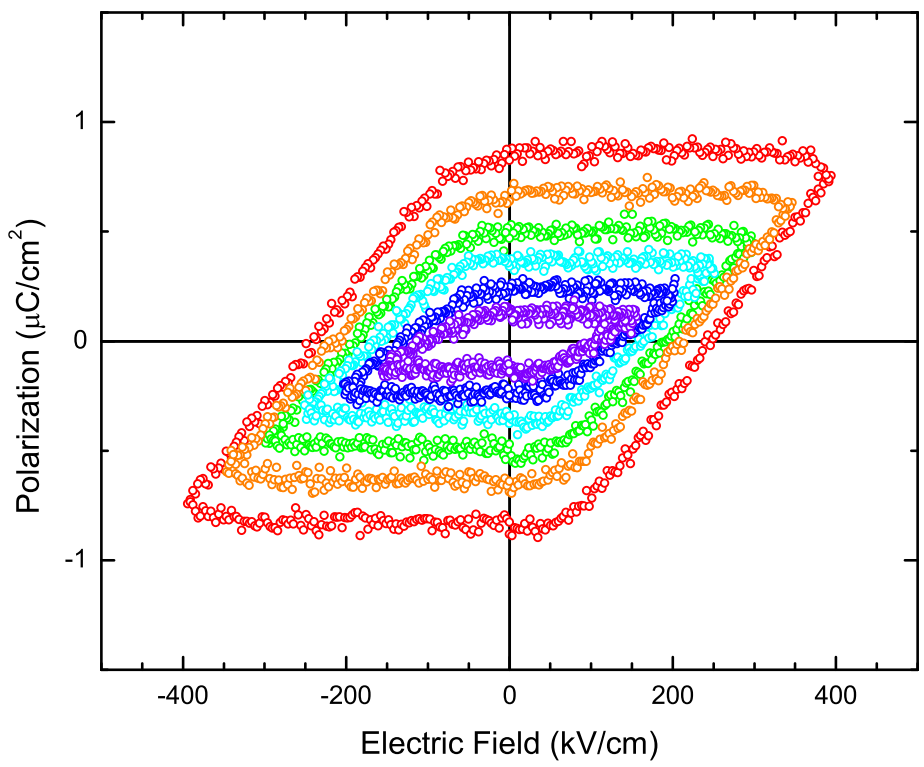

Figure 4.24: Averaged remanent polarization vs. electric field for $\mathrm{BaNiF}_{4}$ film with remanent polarization of $0.9 \mu \mathrm{C} / \mathrm{cm}^{2}$.

samples than was the case for either $\mathrm{BaCoF}_{4}$ or $\mathrm{BaNiF}_{4}$. 


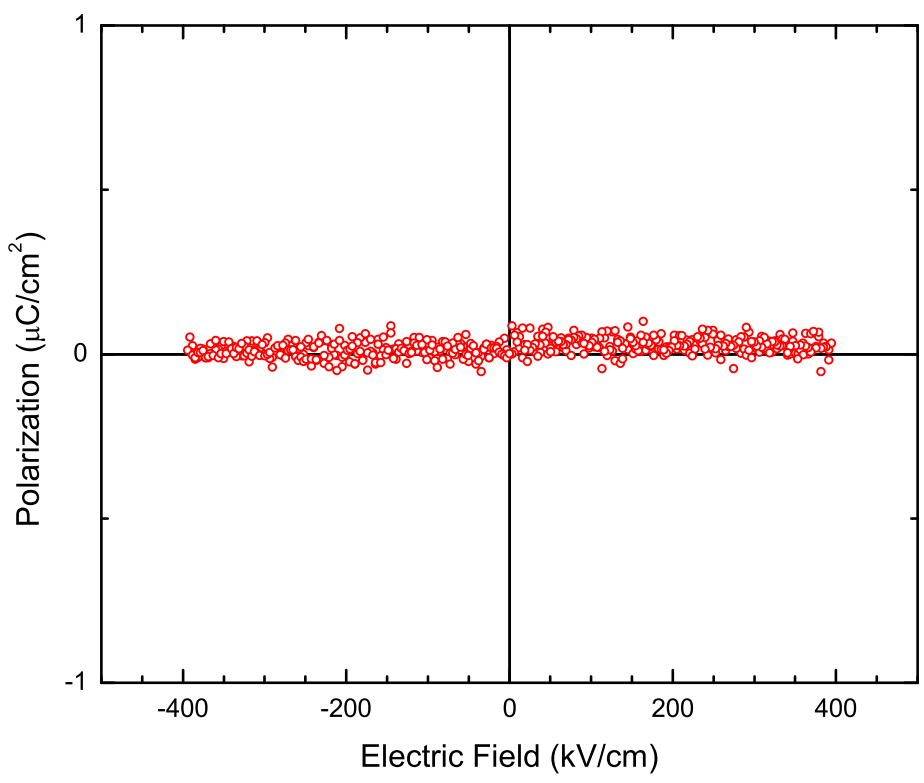

Figure 4.25: Averaged remanent polarization vs. electric field for $\mathrm{BaFeF}_{4}$ film with no ferroelectric switching.

\subsection{Summary}

We have grown the first-ever thin films of the orthorhombic fluorides $\mathrm{BaMF}_{4}(M$ $=\mathrm{Fe}, \mathrm{Co}, \mathrm{Ni}$ ). Our characterization of the magnetic and ferroelectric properties of these films unambiguously confirms multiferroic order, consistent with observations of the bulk, in $\mathrm{BaCoF}_{4}$ and $\mathrm{BaNiF}_{4}$. In addition, previously unobserved ferromagnetic signatures have been detected in all three materials, likely arising due to epitaxial strain. Ferroelectric switching in $\mathrm{BaFeF}_{4}$ has not been confirmed, but neither can our work rule it out, due to limitations on the electric fields that could be applied.While $\mathrm{BaCoF}_{4}$ and $\mathrm{BaNiF}_{4}$ are indeed multiferroic, magneto-electric coupling has not been observed. Ferroelectric hysteresis loops measured on both materials in magnetic fields 
up to $90 \mathrm{kOe}$ were apparently identical to loops taken in zero applied field. However, the noise level in our measurements (see figures 4.22 to 4.24) was sufficiently high that a small magneto-electric effect on the order of $1 \%$ would not be detectable. Therefore, it is not clear whether our films are weakly magneto-electric or not. This issue could provide an avenue for future research into these films. 


\section{Bibliography}

[1] Hill, N.A., Why are there so few magnetic ferroelectrics?, Journal of Physical Chemistry B, 104(29):6694-6709 (2000).

[2] Aharoni, A., Introduction to the theory of ferromagnetism, Oxford University Press, Oxford, New York (2000).

[3] Brown, H.A., Heisenberg ferromagnet with biquadratic exchange, Physical Review $B, 4(1): 115-\&(1971)$.

[4] Blundell, S., Magnetism in condensed matter, Oxford University Press, Oxford, New York (2001).

[5] Dzyaloshinsky, I., A thermodynamic theory of weak ferromagnetism of antiferromagnetics, Journal of Physics and Chemistry of Solids, 4(4):241-255 (1958).

[6] Moriya, T., Anisotropic superexchange interaction and weak ferromagnetism, Physical Review, 120(1):91-98 (1960).

[7] Anderson, P.W., New approach to the theory of superexchange interactions, Physical Review, 115(1):2-13 (1959).

[8] Nogues, J., Lederman, D., Moran, T.J., and Schuller, I.K., Positive exchange bias in $\mathrm{FeF}_{2}$-Fe bilayers, Physical Review Letters, 76(24):4624-4627 (1996).

[9] Meiklejohn, W.H. and Bean, C.P., New magnetic anisotropy, Physical Review, 102(5):1413-1414 (1956).

[10] Tsang, C., Magnetics of small magnetoresistive sensors, Journal of Applied Physics, 55(6):2226-2231 (1984).

[11] Nogues, J. and Schuller, I.K., Exchange bias, Journal of Magnetism and Magnetic Materials, 192(2):203-232 (1999).

[12] Shi, H.T., Lederman, D., O’Donovan, K.V., and Borchers, J.A., Exchange bias and enhancement of the Néel temperature in thin $\mathrm{NiF}_{2}$ films, Physical Review $B, \mathbf{6 9}(21)$ (2004). 
[13] Radu, F., Fundamental aspects of exchange bias effect in $A F / F$ bilayers and multilayers, Ph.D. thesis, Ruhr-University Bochum (2005).

[14] Meiklejohn, W.H. and Bean, C.P., New magnetic anisotropy, Physical Review, 105(3):904-913 (1957).

[15] Meiklejohn, W.H., Exchange anisotropy - a review, Journal of Applied Physics, 33(3):1328-\& (1962).

[16] Stoner, E.C. and Wohlfarth, E.P., A mechanism of magnetic hysteresis in heterogeneous alloys, Philosophical Transactions of the Royal Society of London Series A-Mathematical and Physical Sciences, 240(826):599-642 (1948).

[17] Berkowitz, A.E. and Takano, K., Exchange anisotropy - a review, Journal of Magnetism and Magnetic Materials, 200(1-3):552-570 (1999).

[18] Néel, L., Etude theorique - du couplage ferro-antiferromagnetique dans les couch minces, Annales de Physique, 2(2):61-\& (1967).

[19] Mauri, D., Siegmann, H.C., Bagus, P.S., and Kay, E., Simple-model for thin ferromagnetic-films exchange coupled to an antiferromagnetic substrate, Journal of Applied Physics, 62(7):3047-3049 (1987).

[20] Malozemoff, A.P., Random-field model of exchange-anisotropy at rough ferromagnetic-antiferromagnetic interfaces, Physical Review B, 35(7):3679-3682 (1987).

[21] Miltenyi, P., Gierlings, M., Keller, J., Beschoten, B., Guntherodt, G., Nowak, U., and Usadel, K.D., Diluted antiferromagnets in exchange bias: Proof of the domain state model, Physical Review Letters, 84(18):4224-4227 (2000).

[22] Beckmann, B., Nowak, U., and Usadel, K.D., Asymmetric reversal modes in ferromagnetic/antiferromagnetic multilayers, Physical Review Letters, 91(18) (2003).

[23] Kiwi, M., Exchange bias theory, Journal of Magnetism and Magnetic Materials, 234(3):584-595 (2001).

[24] Lines, M., Principles and applications of ferroelectrics and related materials, Clarendon Press Oxford University Press, Oxford, New York (2001).

[25] Uchino, K., Ferroelectric devices, CRC Press, Boca Raton (2010).

[26] Fiebig, M., Revival of the magnetoelectric effect, Journal of Physics D-Applied Physics, 38(8):R123-R152 (2005). 
[27] Mott, N., The theory of the properties of metals and alloys, Dover publ, New York (1958).

[28] Mott, N.F. and H, W.H., Resistance and thermoelectric properties of the transition metals, Proc. Roy. Soc., A 156:368-382 (1936).

[29] Thomson, W., On the electro-dynamic qualities of metals: effects of magnetization on the electric conductivity of nickel and iron, Proc. Roy. Soc., 8:546-550 (1857).

[30] McGuire, T.R. and Potter, R.I., Anisotropic magnetoresistance in ferromagnetic 3d alloys, IEEE Transactions on Magnetics, 11(4):1018-1038 (1975).

[31] Smit, J., Magnetoresistance of ferromagnetic metals and alloys at low temperatures, Physica, 17(6):612-627 (1951).

[32] Berger, L., Influence of spin-orbit interaction on transport processes in ferromagnetic nickel alloys in presence of degerneracy of 3d band, Physica, 30(6):1141-\& (1964).

[33] Baibich, M.N., Broto, J.M., Fert, A., Vandau, F.N., Petroff, F., Eitenne, P., Creuzet, G., Friederich, A., and Chazelas, J., Giant magnetoresistance of (001)Fe/(001)Cr magnetic superlattices, Physical Review Letters, 61(21):24722475 (1988).

[34] Hirota, E., Giant magneto-resistance devices, Springer, Berlin, New York (2002).

[35] Moodera, J.S., Hao, X., Gibson, G.A., and Meservey, R., Electron-spin polarization in tunnel-junctions in zero applied field with ferromagnetic EuS barriers, Physical Review Letters, 61(5):637-640 (1988).

[36] Itoh, H., Theory of tunnel magnetoresistance and spin filter effect in magnetic tunnel junctions, Journal of Physics D-Applied Physics, 40(5):1228-1233 (2007), 19th International Colloquium on Magnetic Films and Surfaces (ICMFS 2006), Sendai, Japan, AUG 14-18, 2006.

[37] Humphry, K.J., Stamps, R.L., and Folks, L., Effect of atomic disorder on transport through magnetic tunnel junctions, Journal of Applied Physics, 93(10, 2):7522-7524 (2003), 47th Annual Conference on Magnetism and Magnetic Materials, Tampa, Florida, NOV 11-15, 2002.

[38] Herman, M., Molecular beam epitaxy fundamentals and current status, Springer Berlin Heidelberg, Berlin, Heidelberg (1989).

[39] Ichimiya, A., Reflection high-energy electron diffraction, Cambridge University Press, Cambridge (2010). 
[40] Feldman, L., Fundamentals of surface and thin film analysis, North-Holland, New York (1986).

[41] Henini, M., Molecular beam epitaxy from research to mass production, Elsevier, Amsterdam (2012).

[42] SreeHarsha, K.S., Principles of physical vapor deposition of thin films, Elsevier, Amsterdam, Boston, London (2006).

[43] Smith, D., Thin-film deposition: principles and practice, McGraw-Hill, New York (1995).

[44] Mattox, D.M., Handbook of physical vapor deposition (PVD) processing, William Andrew Elsevier Science distributor, Norwich, N.Y., Oxford (2010).

[45] Sze, S.M., Semiconductor devices, physics and technology, Wiley, Hoboken, N.J (2012).

[46] AZ 5200 positive photoresists spec sheet, Merck Chemical.

[47] Harman, G., Wire bonding in microelectronics, McGraw-Hill, New York (2010).

[48] Braun, W., Applied RHEED reflection high-energy electron diffraction during crystal growth, Springer Berlin, Berlin (2013).

[49] Omar, M., Elementary solid state physics: principles and applications, AddisonWesley Pub. Co, Reading, Mass (1975).

[50] Ashcroft, N., Solid state physics, Holt, Rinehart and Winston, New York (1976).

[51] Giacovazzo, C., Fundamentals of crystallography, Oxford University Press, Oxford, New York (2002).

[52] Parratt, L.G., Structure of thin evaporated copper films by total reflection of x-rays, Physical Review, 99(5):1635 (1955).

[53] Gibaud, A. and Hazra, S., X-ray reflectivity and diffuse scattering, Current Science, 78(12):1467-1477 (2000).

[54] Chikazumi, S., Physics of ferromagnetism, Oxford University Press, Oxford, New York (2009).

[55] Annett, J., Superconductivity, superfluids, and condensates, Oxford University Press, Oxford, New York (2004).

[56] Clarke, J., The SQUID handbook, Wiley-VCH, Weinheim (2003). 
[57] Sawyer, C.B. and Tower, C.H., Rochelle salt as a dielectric, Physical Review, 35(3):0269-0273 (1930).

[58] Ferroelectric test system manual, Radiant Technologies Inc. (2010).

[59] Scott, J.F., Ferroelectrics go bananas, Journal of Physics-Condensed Matter, 20(2) (2008).

[60] Tsui, D.C., Dietz, R.E., and Walker, L.R., Multiple magnon excitationin NiO by electron tunneling, Physical Review Letters, 27(25):1729-\& (1971).

[61] Yang, F.Y. and Chien, C.L., Spiraling spin structure in an exchange-coupled antiferromagnetic layer, Physical Review Letters, 85(12):2597-2600 (2000).

[62] Leung, C.W. and Blamire, M.G., Interaction between exchange-bias systems in $\mathrm{Ni}_{80} \mathrm{Fe}_{20} / \mathrm{Fe}_{50} \mathrm{Mn}_{50} /$ Co trilayers, Physical Review B, 72(5) (2005).

[63] Sankaranarayanan, V.K., Yoon, S.M., Kim, D.Y., Kim, C.O., and Kim, C.G., Exchange bias in NiFe/FeMn/NiFe trilayers, Journal of Applied Physics, 96(12):7428-7434 (2004).

[64] Bjorck, M. and Andersson, G., GenX: an extensible x-ray reflectivity refinement program utilizing differential evolution, Journal of Applied Crystallography, 40(6):1174-1178 (2007).

[65] Munbodh, K., Perez, F.A., Keenan, C., Lederman, D., Zhernenkov, M., and Fitzsimmons, M.R., Effects of hydrogen/deuterium absorption on the magnetic properties of Co/Pd multilayers, Physical Review B, 83(9) (2011).

[66] Schrag, B., Anguelouch, A., Ingvarsson, S., Xiao, G., Lu, Y., Trouilloud, P.L., Gupta, A., Wanner, R.A., Gallagher, W.J., Rice, P., and Parkin, S.S.P., Néel "orange-peel" coupling in magnetic tunneling junction devices, Applied Physics Letters, 77(15):2373-2375 (2000).

[67] Moritz, J., Garcia, F., Toussaint, J.C., Dieny, B., and Nozieres, J.P., Orange peel coupling in multilayers with perpendicular magnetic anisotropy: Application to (Co/Pt)-based exchange-biased spin-valves, Europhysics Letters, 65(1):123-129 (2004).

[68] Gredig, T., Krivorotov, I.N., and Dahlberg, E.D., Magnetization reversal in exchange biased $\mathrm{Co} / \mathrm{CoO}$ probed with anisotropic magnetoresistance, Journal of Applied Physics, 91(10, 2):7760-7762 (2002), 46th Annual Conference on Magnetism and Magnetic Materials, Seattle, Washington, NOV 12-16, 2001.

[69] Grimsditch, M., Hoffmann, A., Vavassori, P., Shi, H.T., and Lederman, D., Exchange-induced anisotropies at ferromagnetic-antiferromagnetic interfaces above and below the Néel temperature, Physical Review Letters, 90(25) (2003). 
[70] Khan, M.Y., Wu, C.B., and Kuch, W., Pinned magnetic moments in exchange bias: Role of the antiferromagnetic bulk spin structure, Physical Review B, 89(9) (2014).

[71] Morales, R., Li, Z.P., Olamit, J., Liu, K., Alameda, J.M., and Schuller, I.K., Role of the Antiferromagnetic Bulk Spin Structure on Exchange Bias, Physical Review Letters, 102(9) (2009).

[72] Castro, I.L., Nascimento, V.P., Passamani, E.C., Takeuchi, A.Y., Larica, C., Tafur, M., and Pelegrini, F., The role of the (111) texture on the exchange bias and interlayer coupling effects observed in sputtered $\mathrm{NiFe} / \mathrm{IrMn} / \mathrm{Co}$ trilayers, Journal of Applied Physics, 113(20) (2013).

[73] Jungbauer, M., Huehn, S., Michelmann, M., Goering, E., and Moshnyaga, V., Exchange bias in $\mathrm{La}_{0.7} \mathrm{Sr}_{0.3} \mathrm{MnO}_{3} / \mathrm{SrMnO}_{3} / \mathrm{La}_{0.7} \mathrm{Sr}_{0.3} \mathrm{MnO}_{3}$ trilayers, Journal of Applied Physics, 113(17) (2013), 12th Joint MMM-Intermag Conference, Chicago, IL, JAN 14-18, 2013.

[74] Xu, Y., Ma, Q., Cai, J.W., and Sun, L., Evidence of bulk antiferromagnet spin rearrangement during ferromagnetic layer reversal in a double exchange bias sandwich, Physical Review B, 84(5) (2011).

[75] Choi, H.C., You, C.Y., Kim, K.Y., Lee, J.S., Shim, J.H., and Kim, D.H., Antiferromagnetic layer thickness dependence of noncollinear uniaxial and unidirectional anisotropies in $\mathrm{NiFe} / \mathrm{FeMn} / \mathrm{CoFe}$ trilayers, Physical Review B, 81(22) (2010).

[76] Ambrose, T. and Chien, C.L., Magnetic-properties of exchange-coupled NiFe/CoO/NiFe trilayers, Applied Physics Letters, 65(15):1967-1969 (1994).

[77] Kim, K.Y., Kim, J.W., Choi, H.C., Teichert, A., You, C.Y., Park, S.k., Shin, S.C., and Lee, J.S., Long-range interlayer-coupled magnetization reversal mediated by the antiferromagnetic layer in $\mathrm{Py} / \mathrm{FeMn} / \mathrm{CoFe}$ trilayers, Physical Review $B, \mathbf{8 4}(14)(2011)$.

[78] Nowak, U., Usadel, K.D., Keller, J., Miltenyi, P., Beschoten, B., and Guntherodt, G., Domain state model for exchange bias. I. Theory, Physical Review $B, \mathbf{6 6}(1)(2002)$.

[79] Borisov, P., private communication.

[80] DiDomenico, M., Eibschütz, M., Guggeheim, H.J., and Camlibel, I., Dielectric behavior of ferroelectric $\mathrm{Ba}_{\mathrm{MF}}$ above room temperature, Solid State Communications, 7(16):1119-\& (1969). 
[81] Keve, E.T., Abrahams, S.C., and Bernstein, J.L., Ferroelectric paraelastic paramagnetic barium cobalt fluoride, $\mathrm{BaCoF}_{4}$, crystal structure, Journal of Chemical Physics, 53(8):3279+ (1970).

[82] Cox, D.E., Eibschütz, M., Guggenheim, H.J., and HOLMES, L., Neutron diffraction study of magnetic structure of $\mathrm{BaNiF}_{4}$, Journal of Applied Physics, 41(3):943-\& (1970).

[83] Eibschütz, M. and Guggenheim, H.J., Antiferromagnetic-piezoelectric crystals: $\mathrm{Ba}_{\mathrm{FF}_{4}}(M=\mathrm{Mn}, \mathrm{Fe}$, Co and Ni), Solid State Communications, 6(10):737-\& (1968).

[84] Eibschütz, M., Guggenheim, H.J., Wemple, S.H., Camlibel, I., and DiDomenico, M., Ferroelctricity in $\mathrm{BaM}^{2+} \mathrm{F}_{4}$, Physics Letters A, A 29(7):409-\& (1969).

[85] Scott, J.F., Mechanisms of dielectric anomalies in $\mathrm{BaMnF}_{4}$, Physical Review B, 16(5):2329-2331 (1977).

[86] Eibschütz, M., Guggenheim, H.J., Cox, D.E., and Holmes, L., Magnetic structure of 2-dimensional antiferromagnet $\mathrm{BaCoF}_{4}$, Physical Review B, 6(7):2677-\& (1972).

[87] Scott, J.F., Phase-transitions in $\mathrm{BaMnF}_{4}$, Reports on Progress in Physics, 42(6):1055-\& (1979).

[88] Glass, A.M., Lines, M.E., Eibschütz, M., Hsu, F.S.L., and Guggenheim, H.J., Observation of anomalous pyroelectric behavior in $\mathrm{BaNiF}_{4}$ due to cooperative magnetic singularity, Communications on Physics, 2(4):103-107 (1977).

[89] Kleemann, W., Schafer, F.J., and Nouet, J., Linear magnetic birefringence and double-excitonic transitions of the two-dimensional antiferromagnet $\mathrm{BaNiF}_{4}$, Journal of Physics C-Solid State Physics, 14(30):4447-4461 (1981).

[90] Ederer, C. and Spaldin, N.A., Origin of ferroelectricity in the multiferroic barium fluorides $\mathrm{BaMF}_{4}$ : A first principles study, Physical Review B, 74(2) (2006).

[91] Ederer, C. and Spaldin, N.A., Electric-field-switchable magnets: The case of $\mathrm{BaNiF}_{4}$, Physical Review B, 74(2) (2006).

[92] Scott, J.F. and Blinc, R., Multiferroic magnetoelectric fluorides: why are there so many magnetic ferroelectrics?, Journal of Physics-Condensed Matter, 23(11) (2011).

[93] Simeonov, K. and Lederman, D., Surface structure of (112̄0) $\mathrm{Al}_{2} \mathrm{O}_{3}$ single crystals after high temperature annealing, Surface Science, 603(1):232-236 (2009). 
[94] Aizawa, K., Moriwaki, M., Ichiki, T., Tokumitsu, E., and Ishiwara, H., Growth and crystallinity of ferroelectric $\mathrm{BaMgF}_{4}$ films on (111)-oriented Pt films, Japanese Journal of Applied Physics Part 2-Letters, 36(2B):L234-L237 (1997).

[95] McChesney, J., Hetzer, M., Shi, H., Charlton, T., and Lederman, D., Growth study of epitaxial $\mathrm{Fe}_{x} \mathrm{Zn}_{1-x} \mathrm{~F}_{2}$ thin films, Journal of Materials Research, 16(6):1769-1775 (2001).

[96] Marti, X., Skumryev, V., Ferrater, C., Garcia-Cuenca, M.V., Varela, M., Sanchez, F., and Fontcuberta, J., Emergence of ferromagnetism in antiferromagnetic TbMnO3 by epitaxial strain, Applied Physics Letters, 96(22) (2010).

[97] Bazhan, A.N. and Bazan, C., Weak ferromagnetism in $\mathrm{CoF}_{2}$ and $\mathrm{NiF}_{2}$, Soviet Journal of Experimental and Theoretical Physics, 69(5):1768-1781 (1975).

[98] Garcia-Castro, A.C. and Romaro, A., private communication. 